\title{
A slitless spectroscopic survey for quasars near quasars ${ }^{\star}, \star \star$
}

\author{
G. Worseck ${ }^{1}$, L. Wisotzki ${ }^{1}$, and F. Selman ${ }^{2}$ \\ 1 Astrophysikalisches Institut Potsdam, An der Sternwarte 16, 14482 Potsdam, Germany \\ e-mail: gworseck@aip.de \\ 2 European Southern Observatory, Alonso de Cordova 3107, Casilla 19001, Vitacura, Santiago, Chile \\ Received 7 May 2008 / Accepted 10 June 2008
}

\section{ABSTRACT}

\begin{abstract}
We present the results of the "Quasars near Quasars" (QNQ) survey, a CCD-based slitless spectroscopic survey for faint $V \lesssim$ 22 quasars at $1.7 \lesssim z \lesssim 3.6$ on $1826.2 \times 33.5$ fields centred on bright quasars at $2.76<z<4.69$. In total 169 quasar candidates with emission lines were selected from the extracted flux-calibrated spectra on the basis of well-defined automatic selection criteria followed by visual inspection and verification. With follow-up spectroscopy of 81 candidates that were likely to reside at $z>1.7$ we were able to confirm 80 new quasars at $0.580 \leq z \leq 3.586$ on 16 of our fields. 64 of the newly discovered quasars are located at $z>1.7$. The overall high success rate implies that most of the remaining 88 candidates are quasars as well, although the majority of them likely resides at $z<1.7$ on the basis of the observed line shapes and strengths. Due to the insufficient depth of the input source catalogues needed for extraction of the slitless spectra our survey is not well defined in terms of limiting magnitude for faint $2.5 \lessgtr z \lesssim 3.6$ quasars whose Ly $\alpha$ emission is detectable well beyond $V=22$, albeit at a continuum $S / N \lessgtr 1$. While not useful for characterising the evolving space density of quasars, our sample provides many new closely spaced quasar sightlines around intensely studied quasars for further investigations on the three-dimensional distribution of the intergalactic medium.
\end{abstract}

Key words. surveys - galaxies: quasars: general - galaxies: intergalactic medium - cosmology: large-scale structure of Universe

\section{Introduction}

Recent large optical surveys, such as the Sloan Digital Sky Survey (SDSS) and the 2dF QSO Redshift Survey (2QZ), have revealed thousands of previously unknown quasars selected on the basis of their broadband optical colours (Schneider et al. 2007; Croom et al. 2004). Colour selection is efficient if quasar candidates are well separated from normal stars in multidimensional colour space, most notably at $z \lessgtr 2.2$ (UV excess) and at $z \gtrsim 3.5$. However, even optical multicolour surveys are systematically incomplete at $2.5 \lessgtr z \lesssim 3.5$, where the colours of quasars and stars are similar (e.g. Warren et al. 1991; Richards et al. 2002a). Incompleteness in this redshift range can be significantly reduced by a better tracing of the spectral energy distributions with additional filters, e.g. by incorporating mediumband filters as in the COMBO-17 survey (Wolf et al. 2003).

Alternatively, slitless spectroscopy is a particularly efficient way to find quasars at redshifts $z \gtrsim 2$ because of the prominent Ly $\alpha$ emission line redshifted into the optical wavelength regime. Early surveys generated hundreds of quasars by visual scanning of objective-prism photographic plates for emission-line objects (e.g. Osmer \& Smith 1980; Crampton et al. 1985). Subjecting such plates to digitisation with fast measuring machines made it possible to employ the automated selection of quasar candidates (Clowes et al. 1984; Hewett et al. 1985) and to build substantial quasar samples at redshifts $0 \lesssim z \lesssim 3.2$ (Hewett et al. 1995; Wisotzki et al. 2000). The systematic CCD-based slitless survey for $2.7 \lessgtr z \lesssim 4.8$ quasars by Schneider et al. (1994) was among

\footnotetext{
* Based on observations collected at the European Southern Observatory, Chile (Proposals 70.A-0425, 074.A-0273 and 075.A-0141). Data taken under proposals 68.A-0330 and 70.A-0384 were obtained from the ESO Science Archive.

$\star \star$ Table 2 and Appendices A-C are only available in electronic form at http://www . aanda.org
}

the first to quantify the declining space density of high-redshift quasars (Schmidt et al. 1995).

Apparent pairs or close groups of high-redshift quasars are very attractive targets to study the three-dimensional distribution of the intergalactic medium (IGM). But high-resolution studies have so far been limited to a small number of suitable groups of bright quasars (e.g. D'Odorico et al. 2002, 2006). Going fainter than $V \sim 19$ immediately limits the achievable spectral resolution and $S / N$. A possible compromise lies in combining highresolution spectra of bright quasars with lower resolution, lower $S / N$ data of fainter quasars in the surroundings. Pichon et al. (2001) argued that one can significantly improve the recovery of the 3-dimensional topology of the IGM this way.

Although most known quasars were colour-selected from either SDSS or 2QZ, these surveys produced relatively few useful quasar groups because of the reduced selection efficiency at $z \gtrsim 2.5$ combined with a bright magnitude limit. In fact, most well-studied close groups of quasars at $z>2$ were found by slitless spectroscopy. Follow-up spectroscopy of candidates by Bohuski \& Weedman (1979) revealed $13 z>1.5$ quasars on $2.1 \mathrm{deg}^{2}$ (Jakobsen \& Perryman 1992) with two showing correlated complex intergalactic C IV absorption at $1.48<z<2.15$, indicative of an elongated superstructure extending over 17!9 on the sky (e.g. Jakobsen et al. 1986; Dinshaw \& Impey 1996). Another group discovered by Sramek \& Weedman (1978) contains now 6 QSOs at $2.49 \leq z \leq 3.45$ within a radius of $20^{\prime}$ around Q $1623+2653$, all of which are bright enough for correlation studies of the IGM (e.g. Crotts \& Fang 1998). Williger et al. (1996) reported 25 quasars at $1.5 \lesssim z \lesssim 3.4$ within a $\sim 1 \mathrm{deg}^{2}$ region and used these to reveal large-scale structure in the IGM (Williger et al. 2000; Liske et al. 2000). At lower redshifts, slitless surveys revealed large associations of quasars at similar redshift (Crampton et al. 1990; Clowes et al. 1999). 
Groups of quasars, or more generally speaking, active galactic nuclei, have also been discovered by recent deep multiwavelength surveys in selected fields, such as in the Chandra Deep Fields North (Barger et al. 2003; Cowie et al. 2004) and South (Szokoly et al. 2004; Wolf et al. 2004), the Marano field (Zitelli et al. 1992; Krumpe et al. 2007), or the COSMOS field (Prescott et al. 2006; Trump et al. 2007). However, the majority of the AGN thus found are too faint for follow-up studies at a spectral resolution allowing for meaningful IGM studies.

Complementary to slitless spectroscopy, quasar pairs can be found from similarities in multi-colour space. Using this approach, Hennawi et al. (2006b) confirmed 40 new associated and 73 projected quasar pairs with separations $\vartheta<1^{\prime}$ from a sample of faint $i \lesssim 21$ quasar pair candidates selected via SDSS photometry. Follow-up spectra revealed transverse clustering of optically thick absorption systems near foreground quasars (Hennawi et al. 2006a; Hennawi \& Prochaska 2007).

Targeted deep surveys of sky regions around well-studied high-redshift quasars are rare. An exception are many of the fields selected for the Lyman-break galaxy survey by Steidel et al. (2003) which were centred on bright quasars to correlate the galaxies with the intergalactic absorption along the sightline (Adelberger et al. 2005). Here we describe a systematic search for apparent quasar groups at $1.7 \lessgtr z \lesssim 3.6$ in the southern hemisphere, targeting fields centred on known bright $z>2.7$ quasars that had been observed at high resolution with the UV-Visual Echelle Spectrograph (UVES) at the VLT. We already reported results for two special fields (Worseck \& Wisotzki 2006; Worseck et al. 2007, hereafter Papers I and II, respectively). The present paper is devoted to present the entire survey. In Sect. 2 we describe the slitless spectroscopic survey observations. Section 3 outlines the automatic reduction pipeline developed for these data. Section 4 describes the semi-automatic selection of quasar candidates. We report on the follow-up slit spectroscopy of candidates in Sect. 5, followed by a brief discussion of the properties of confirmed quasars and the remaining candidates (Sect. 6). In Sect. 7 we present the resulting quasar groups and discuss the efficiency and completeness of our survey. We conclude in Sect. 8.

\section{Survey observations}

The survey was carried out using the ESO Wide Field Imager (WFI, Baade et al. 1999) at the ESO/MPI $2.2 \mathrm{~m}$ Telescope (La Silla) in its slitless spectroscopic mode (Wisotzki et al. 2001). Since this mode has been rarely used we shortly describe its main characteristics.

The WFI is a focal-reducer type camera offering a field of view of $34^{\prime} \times 33^{\prime}$ sampled by a mosaic of $82 \mathrm{k} \times 4 \mathrm{k}$ CCDs with $0 . ' 238 /$ pixel. In the slitless spectroscopic mode a low-resolution grism is placed in the converging beam of the telescope in front of the WFI in order to disperse the light of every object in the field of view. A blue-blazed grism and a red-blazed grism are available. However, the red-blazed grism (R50, dispersion $\sim 7 \AA$ /pixel, blaze wavelength $6000 \AA, \Delta \lambda \sim 50 \AA$ FWHM) has a much higher throughput for 1st-order spectroscopy even in the blue (Wisotzki et al. 2001), rendering the blue-blazed B50 grism almost obsolete. We only used the R50 grism. As both grisms were originally constructed for the prime focus of the ESO $3.6 \mathrm{~m}$ telescope (long decommissioned) and only retrofitted into WFI, the size of the grisms does not fully match that of the WFI field of view. In a 7!2 strip on the left side of the chip mosaic the light passes undispersed, effectively reducing the usable field of view to 6 of the 8 WFI CCDs, or $25^{\prime} \times 33^{\prime}$.

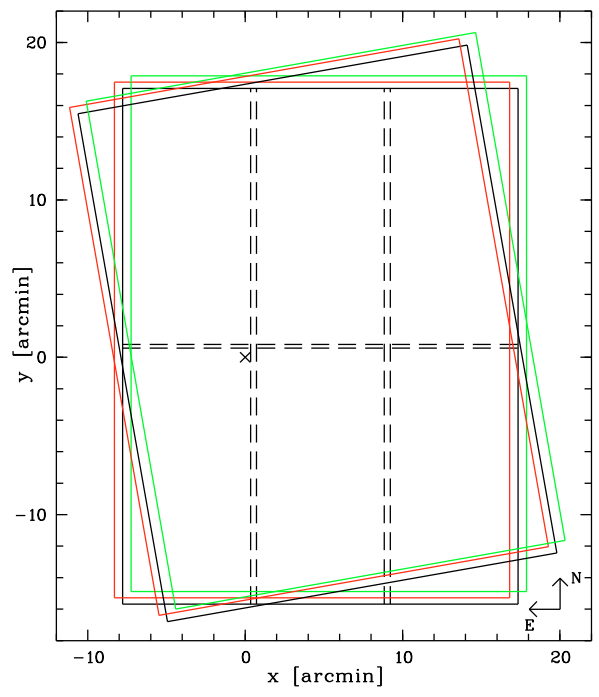

Fig. 1. Schematic view of the WFI dither pattern. The full lines denote the edges of the $25: 1 \times 32$ : 7 field of view in the dithered $600 \mathrm{~s}$ exposures. Dashed lines mark the $62 \mathrm{k} \times 4 \mathrm{k}$ chips with the inter-chip gaps. After rotating the instrument by $10^{\circ}$ about its origin in the focal plane (cross) three further $600 \mathrm{~s}$ exposures were taken.

During two visitor mode runs in October 2002 (5 nights) and February 2003 (3 nights) we observed in total 18 fields centred on bright high-redshift quasars with available UVES spectra in order to find faint quasars in their surroundings. We additionally included the Extended Chandra Deep Field South (ECDFS) to compare our selection with the results of deeper multiwavelength surveys.

The sky transparency during the October 2002 run was variable with some thick cirrus clouds passing occasionally, but mostly the sky was clear. Conditions were clear to photometric in the February 2003 run. The seeing varied over a broad range of values (see Table 1) but was mostly of the order of 1 ". Since the spectral resolution in slitless spectroscopy is given by the seeing, this quantity varied by $\lambda / \Delta \lambda=30-50$. We used the broadband $B$ and $V$ filters of the WFI to lower the effectively undispersed sky background and to reduce the degree of crowding by limiting the length of the spectra. Crowding was further accounted for by taking the spectra in two instrument rotations $\left(0^{\circ}\right.$ and $\left.10^{\circ}\right)$. For all fields (except the one centred on BR 1202-0725) three dithered $600 \mathrm{~s}$ exposures were taken per band and rotation, resulting in a total exposure time of $1 \mathrm{~h}$ per band. Figure 1 illustrates the observing pattern. The dithered exposures produced a contiguous $\sim 26$ ' $2 \times 33$ '.5 field of view per rotation angle. The combination of the R50 grism with the broadband $B$ and $V$ filters resulted in a spectral coverage from the blue sensitivity cutoff at $\sim 4200 \AA$ to $5800 \AA$. An unfiltered spectrum of the low-redshift emissionline galaxy HE 1250-0256 provided the input for an approximate wavelength calibration of the slitless data. The spectrophotometric standard stars HD 49798, LTT 7987 and GD 108 were observed in astronomical twilight for relative flux calibration. Blank sky fields observed during twilight provided flat fields. The $V$ band exposures of the field centred on PKS 0528-250 were retrieved from the ESO Science Archive (PI Vanzi). Table 1 summarises the slitless survey observations.

The nominal surveyed area was $\simeq 4.39 \mathrm{deg}^{2}$. However, due to the two rotation angles of the instrument, the field edges received only $\sim 1 / 2$ of the exposure time. Moreover, spectra located near interchip gaps are affected by dithering. The net exposure time per field is further decreased for some objects due to 
Table 1. Observing log of the slitless survey observations. The columns list the quasar at the field centre with redshift and celestial coordinates, the night of observation, the employed filters, instrument rotations, total exposure time per band and the seeing.

\begin{tabular}{|c|c|c|c|c|c|c|c|c|}
\hline Field & $z$ & $\alpha(\mathrm{J} 2000)$ & $\delta(\mathbf{J} 2000)$ & Night & Filters & Rotations $\left[{ }^{\circ}\right]$ & Exposure [s] & Seeing ["] \\
\hline \multirow[t]{2}{*}{ Q 0000-263 } & 4.125 & $00^{\mathrm{h}} 03^{\mathrm{m}} 22^{\mathrm{s}} .91$ & $-26^{\circ} 03^{\prime} 16^{\prime \prime} 8$ & 03 Oct. 2002 & $B, V$ & 0 & 1800 & $0.7-1.0$ \\
\hline & & & & 04 Oct. 2002 & $B, V$ & 10 & 1800 & $0.9-1.4$ \\
\hline Q 0002-422 & 2.767 & $00^{\mathrm{h}} 04^{\mathrm{m}} 48^{\mathrm{s}} .11$ & $-41^{\circ} 57^{\prime} 28^{\prime \prime} 8$ & 01 Oct. 2002 & $B, V$ & 0,10 & 3600 & $1.3-1.6$ \\
\hline Q 0055-269 & 3.665 & $00^{\mathrm{h}} 57^{\mathrm{m}} 57^{\mathrm{s}} .92$ & $-26^{\circ} 43^{\prime} 14^{\prime \prime} .2$ & 02 Oct. 2002 & $B, V$ & 0,10 & 3600 & $0.9-1.5$ \\
\hline \multirow[t]{3}{*}{ Q 0302-003 } & 3.285 & $03^{\mathrm{h}} 04^{\mathrm{m}} 49^{\mathrm{s}} .86$ & $-00^{\circ} 08^{\prime} 13^{\prime \prime} .4$ & 03 Oct. 2002 & $B, V$ & 0 & 1800 & 0.9 \\
\hline & & & & 03 Oct. 2002 & $B$ & 10 & 1800 & 0.9 \\
\hline & & & & 04 Oct. 2002 & $V$ & 10 & 1800 & $0.9-1.1$ \\
\hline \multirow{3}{*}{ Q 0347-383 } & 3.220 & $03^{\mathrm{h}} 49^{\mathrm{m}} 43^{\mathrm{s}} .68$ & $-38^{\circ} 10^{\prime} 31^{\prime \prime} .3$ & 27 Feb. 2003 & $B, V$ & 10 & 1800 & 1.0 \\
\hline & & & & 27 Feb. 2003 & $V$ & 0 & 1800 & 1.0 \\
\hline & & & & 28 Feb. 2003 & $B$ & 0 & 1800 & 1.9 \\
\hline \multirow[t]{2}{*}{ CTQ 0247} & 3.025 & $04^{\mathrm{h}} 07^{\mathrm{m}} 17.99$ & $-44^{\circ} 10^{\prime} 13^{\prime \prime} 4$ & 30 Sep. 2002 & $B, V$ & 0 & 1800 & $1.3-1.8$ \\
\hline & & & & 01 Oct. 2002 & $B, V$ & 10 & 1800 & $1.4-1.8$ \\
\hline Q 0420-388 & 3.120 & $04^{\mathrm{h}} 22^{\mathrm{m}} 14^{\mathrm{s}} .81$ & $-38^{\circ} 44^{\prime} 52^{\prime \prime} 9$ & 26 Feb. 2003 & $B, V$ & 0,10 & 3600 & 0.8 \\
\hline \multirow[t]{3}{*}{ PKS 0528-250 } & 2.813 & $05^{\mathrm{h}} 30^{\mathrm{m}} 07.96$ & $-25^{\circ} 03^{\prime} 29^{\prime} 9$ & 03 Nov. 2002 & $V$ & 10 & 1800 & 1.1 \\
\hline & & & & 04 Nov. 2002 & $V$ & 0 & 1800 & 1.2 \\
\hline & & & & 28 Feb. 2003 & $B$ & 0,10 & 3600 & 1.2 \\
\hline HE $0940-1050$ & 3.088 & $09^{\mathrm{h}} 42^{\mathrm{m}} 53^{\mathrm{s}} .40$ & $-11^{\circ} 04^{\prime} 25^{\prime \prime} 0$ & 26 Feb. 2003 & $B, V$ & 0,10 & 3600 & 0.8 \\
\hline CTQ 0460 & 3.139 & $10^{\mathrm{h}} 39^{\mathrm{m}} 09^{\mathrm{s}} .51$ & $-23^{\circ} 13^{\prime} 25^{\prime \prime} .7$ & 27 Feb. 2003 & $B, V$ & 0,10 & 3600 & $1.0-1.5$ \\
\hline BR 1117-1329 & 3.958 & $11^{\mathrm{h}} 20^{\mathrm{m}} 10^{\mathrm{s}} .30$ & $-13^{\circ} 46^{\prime} 25^{\prime \prime} 0$ & 28 Feb. 2003 & $B, V$ & 0,10 & 3600 & $>2$ \\
\hline \multirow[t]{2}{*}{ BR $1202-0725$} & 4.690 & $12^{\mathrm{h}} 05^{\mathrm{m}} 23^{\mathrm{s}} .12$ & $-07^{\circ} 42^{\prime} 32^{\prime \prime} .5$ & 26 Feb. 2003 & $B, V$ & 0 & 1800 & 0.8 \\
\hline & & & & 27 Feb. 2003 & $V$ & 10 & 1800 & 1.6 \\
\hline Q $1209+093$ & 3.291 & $12^{\mathrm{h}} 11^{\mathrm{m}} 34^{\mathrm{s}} .95$ & $+09^{\circ} 02^{\prime} 20^{\prime} 9$ & 27 Feb. 2003 & $B, V$ & 0,10 & 3600 & 1.6 \\
\hline Q $1451+123$ & 3.246 & $14^{\mathrm{h}} 54^{\mathrm{m}} 18^{\mathrm{s}} .61$ & $+12^{\circ} 10^{\prime} 54^{\prime \prime} 8$ & 28 Feb. 2003 & $B, V$ & 0,10 & 3600 & $0.8-2.0$ \\
\hline PKS 2126-158 & 3.285 & $21^{\mathrm{h}} 29^{\mathrm{m}} 12^{\mathrm{s}} .18$ & $-15^{\circ} 38^{\prime} 41^{\prime \prime} 0$ & 30 Sep. 2002 & $B, V$ & 0,10 & 3600 & $0.8-1.0$ \\
\hline Q 2139-4434 & 3.214 & $21^{\mathrm{h}} 42^{\mathrm{m}} 25^{\mathrm{s}} .81$ & $-44^{\circ} 20^{\prime} 17^{\prime \prime} .2$ & 03 Oct. 2002 & $B, V$ & 0,10 & 3600 & $1.0-1.5$ \\
\hline HE 2243-6031 & 3.010 & $22^{\mathrm{h}} 47^{\mathrm{m}} 09^{\mathrm{s}} .10$ & $-60^{\circ} 15^{\prime} 45^{\prime \prime} .0$ & 02 Oct. 2002 & $B, V$ & 0,10 & 3600 & $1.0-1.7$ \\
\hline HE $2347-4342$ & 2.885 & $23^{\mathrm{h}} 50^{\mathrm{m}} 34^{\mathrm{s}} .21$ & $-43^{\circ} 25^{\prime} 59^{\prime \prime} 6$ & 04 Oct. 2002 & $B, V$ & 0,10 & 3600 & $0.8-1.2$ \\
\hline
\end{tabular}

contamination by nearby other sources or spectral orders of bright stars (see Fig. 3 below). However, the two instrument rotations ensured that a clean spectrum of almost every object was obtained.

Direct images are necessary for object identification in the slitless data. We primarily relied on images from the Digitised Sky Survey (DSS) $)^{1}$ available for all our fields. For three fields we also employed direct WFI $B V R$ images taken in service mode.

\section{Survey data reduction}

\subsection{Reduction steps}

The slitless data were reduced with a semi-automatic pipeline running under ESO-MIDAS, but largely consisting of our own dedicated software modules. For several parts of the data reduction we could use the extensive toolbox developed for the slitless spectroscopy reduction pipeline of the Hamburg/ESO Survey (HES, Wisotzki et al. 2000). This was supplemented by new software where necessary. Each exposure was reduced separately before combining the extracted spectra. Briefly, the data reduction comprised the following steps:

1. Bias subtraction: bias subtraction was performed by taking the median of the overscan regions of each WFI chip and

1 The Digitized Sky Surveys were produced at the Space Telescope Science Institute under US Government grant NAG W-2166. The images of these surveys are based on photographic data obtained using the Oschin Schmidt Telescope on Palomar Mountain and the UK Schmidt Telescope. The plates were processed into the present compressed digital form with the permission of these institutions. subtracting it from the science exposure. This procedure assured that always the correct bias value was subtracted, since restarts of the electronics were necessary a few times during the nights, altering the bias level.

2. Flat-fielding: the flat-fielding of slitless spectroscopic data is complicated by the fact that in a science exposure a pixel of a slitless spectrum receives the effectively undispersed broadband sky background and nearly monochromatic light from the object, whereas it is exposed only to the undispersed broadband skylight when obtaining the twilight flat field. After some experiments, we obtained good master flat fields by normalising the individual flat field frames by a smoothed 3rd-order polynomial fit along the dispersion direction followed by averaging the normalised images. Flat-fielding of the science data was performed by dividing by the appropriate master flat field.

3. Sky subtraction: the sky background was subtracted using a background image created after masking the spectra using a programme developed for HES. First, the mode of the image was estimated in coarse cells before masking the regions containing spectra by a $\sigma$-clipping algorithm. Subsequently, the average of the unmasked pixels was computed on a finer grid and the variable background was determined by bilinear interpolation. The parameters for creating the masks were varied until the sky-subtracted image was free from artefacts due to over- or undersubtraction near the spectra of bright objects, and the sky residuals were consistent with zero.

4. Astrometric transformation: bright stars on the DSS images were used for an iterative astrometric transformation that yielded the coordinates for the extraction of the spectra. In 


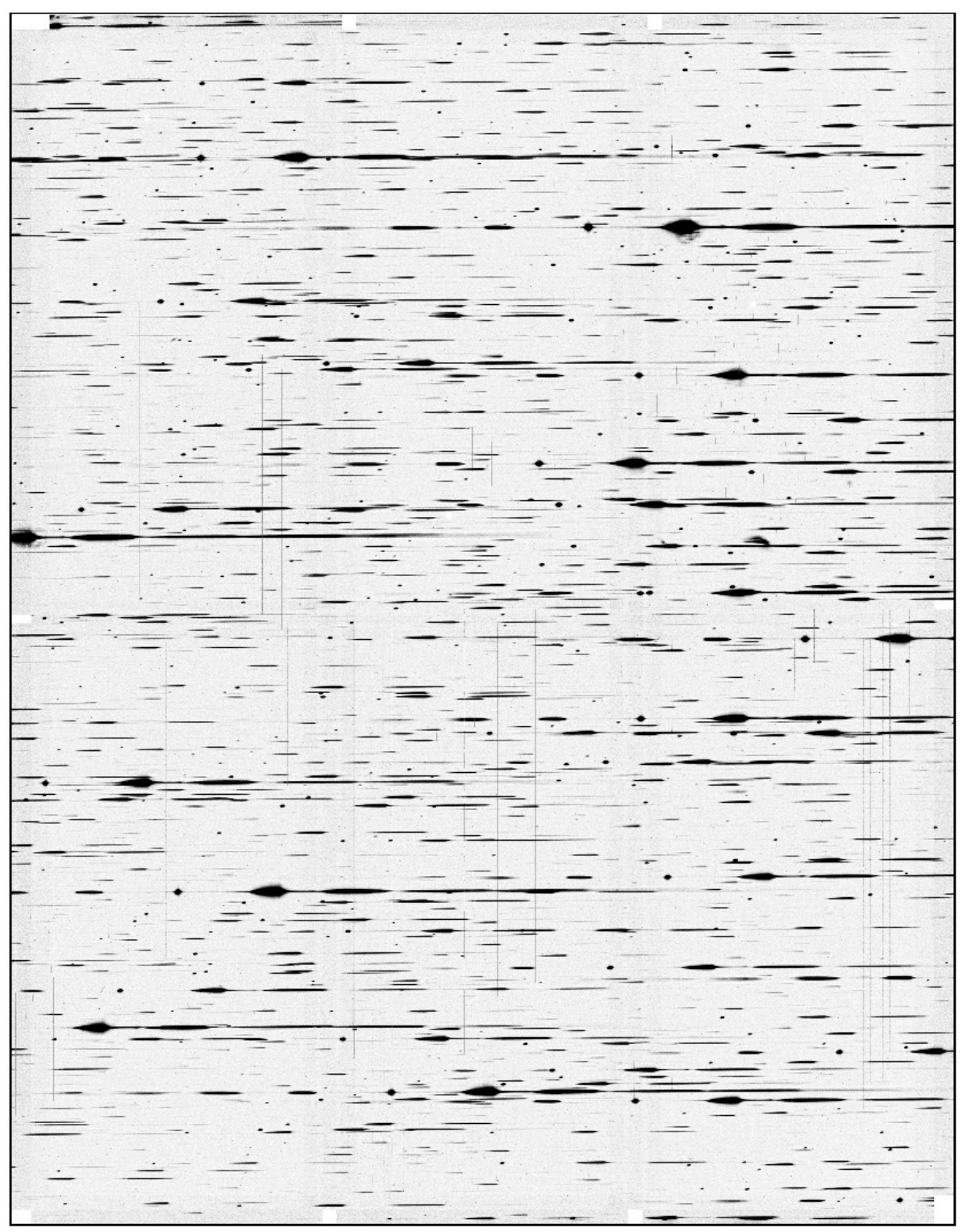

Fig. 2. Flat-fielded sky-subtracted and combined slitless spectroscopic WFI field in the vicinity of HE $0940-1050$. The spectra were taken with the $B$ filter and without rotation (i.e. north is up and east is left on the image). The dispersion runs horizontally from left to right. The three dithered $600 \mathrm{~s}$ exposures were combined to fill the inter-chip gaps. The (linear) intensity of each pixel is weighted by its effective exposure time. The total dimension of the combined image is $26 ! 16 \times 33 ! 53$. an initial step, several objects in each field were visually identified in both direct and spectral images. The cutoff of the object flux at the red end of both filters yielded a welldefined fixpoint for all considered sources. Essentially this was the only reduction step requiring human intervention prior to the selection of candidates. The identified stars defined a preliminary bilinear transformation from the sky to spectral plate coordinates, which was then refined by including several more stars automatically identified in the direct data. Rejecting outliers, the final sets of transformation coefficients were based on $\sim 15-50$ sources per detector chip subfield and provided a mapping between DSS coordinates and nominal red cutoff of the 1 st-order spectral positions with a typical accuracy of \pm 2 pixels (rms).

5. Input source catalogue: the object search routine was used to generate deep source lists of the DSS images. We required an object to have at least 3 pixels $4 \sigma$ above the background level. The lists also contained objects located at the field edges that were not recorded in every exposure due to dithering or instrument rotation. The corresponding coordinates of the sources on the slitless images were derived from the astrometric transformation. For most of the fields the limiting magnitude of the source catalogues was $V_{\text {lim }} \simeq 21$ (Sect. 3.2).

6. Masking of spectral orders and defects: in contrast to an objective prism, a grism produces several spectral orders. Figure 2 shows a combined $26.2 \times 33.5$ slitless spectroscopic WFI image centred on the quasar HE 0940-1050 taken in the $B$ filter. More than $60 \%$ of the flux is concentrated in the +1 st order spectra, with most of the remaining flux going into the 2nd and 0th orders (Wisotzki et al. 2001). Thus, for faint objects only these orders are easily visible in the data. Inevitably, brighter stars produce also higher spectral orders (and negative ones, streching to the left in Fig. 2). In all cases, overlaps between different orders of different objects is in danger of producing undesirable artefacts; a particular nuisance for our programme are the blob-like zero-order images that superposed on other spectra will very much resemble emission lines. We developed a masking scheme combined with the extraction (next step) where all pixels affected by unwanted spectral orders from another object (depending on the magnitude of the recorded object) were flagged in the corresponding variance frame. Figure 3 shows a small section from a spectral image together with its corresponding 


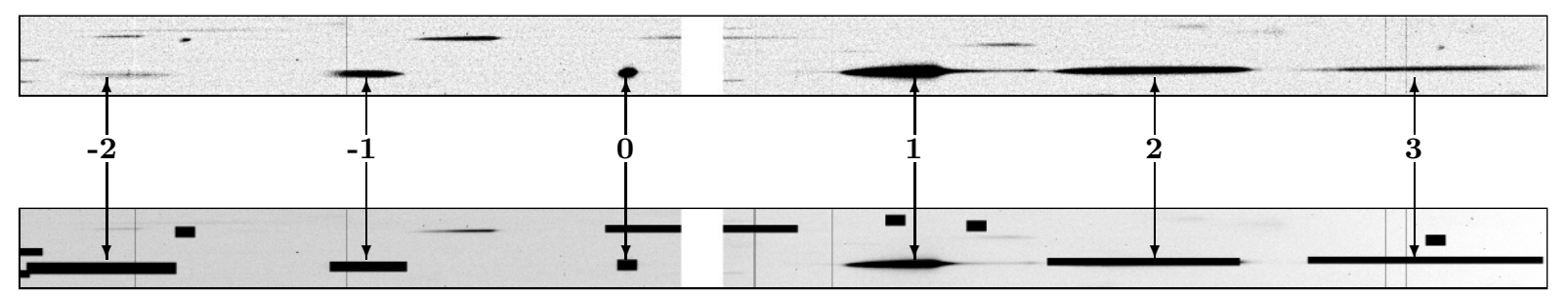

Fig. 3. Masking of unwanted spectral orders. The upper panel shows a $850^{\prime \prime} \times 45^{\prime \prime}$ region of a sky-subtracted $600 \mathrm{~s} B$ band exposure of the field around HE 0940-1050. A bright star in this region generates the indicated different spectral orders in the slitless exposure that may contaminate 1 st-order spectra of nearby sources. The white stripe marks a vertical inter-chip gap. The lower panel displays the corresponding region of the variance image. Black rectangles show masked spectral orders of the bright star and other objects. Dark vertical stripes correspond to masked bad columns on the chips.

variance frame where all spectral orders of an object contaminating other spectra were masked. Also included in the masks were all detector bad pixels and columns as well as cosmic ray hits.

7. Optimal extraction: the slitless spectra of the sources in the input catalogues of each exposure were extracted assuming a Gaussian spatial profile with predefined centroid and full width at half maximum (FWHM). We found the bright stars used for the coordinate transformation to be reliable tracers of the centroid of a spectrum and its FWHM. The centroid vector is usually slightly tilted with respect to the detector columns due to imperfect alignment of the dispersion axis with the CCD rows. The FWHM is primarily set by the seeing, but the grism optics make it increase slightly from east to west over the field of view. All objects in the input catalogue were then extracted with the pre-determined centroid and FWHM vectors of each exposure, maximising the signal-tonoise ratio $(S / N)$ of faint objects. Along with each spectrum, a $1 \sigma$ noise array was extracted. The extracted spectra of all $600 \mathrm{~s}$ exposures were adjusted to a common counts scale to correct for possible sky transparency variations, before averaging them by weighting with their inverse pixel variances. The masking usually (but not always) ensured that overlaps with other orders were adequately reflected in the pixel variances, so that affected objects were generally free of overlap artefacts after averaging the spectra taken at the two rotator angles.

8. Wavelength calibration: Gaussian fits to emission lines of the high- $S / N$ spectrum of the low-redshift emission line galaxy HE 1250-0256 yielded an approximate wavelength scale with a linear dispersion of $\simeq 6.7 \AA /$ pixel. Both the dispersion and the wavelength zero point (as determined by the astrometric transformation) change somewhat over the field of view due to optical distortions induced by the grism (Wisotzki et al. 2001). These variations ultimately limited the accuracy by which wavelengths could be measured in any given spectrum to roughly $\Delta \lambda \simeq \pm 15 \AA$.

9. Flux calibration: observations of three spectrophotometic standard stars yielded the instrument sensitivity curves of the spectra taken in the two filters (Fig. 4). Flux calibration of the spectra was achieved by dividing the extracted spectra by the appropriate sensitivity curve scaled to the nominal exposure time of $600 \mathrm{~s}$. Because of changes in sky transparency during the first observing run and because of the long intervals between standard star observations, the flux scale should be considered as relative, although we found good agreement between the published magnitudes and the integrated slitless spectroscopic magnitudes of the known quasars in the fields.

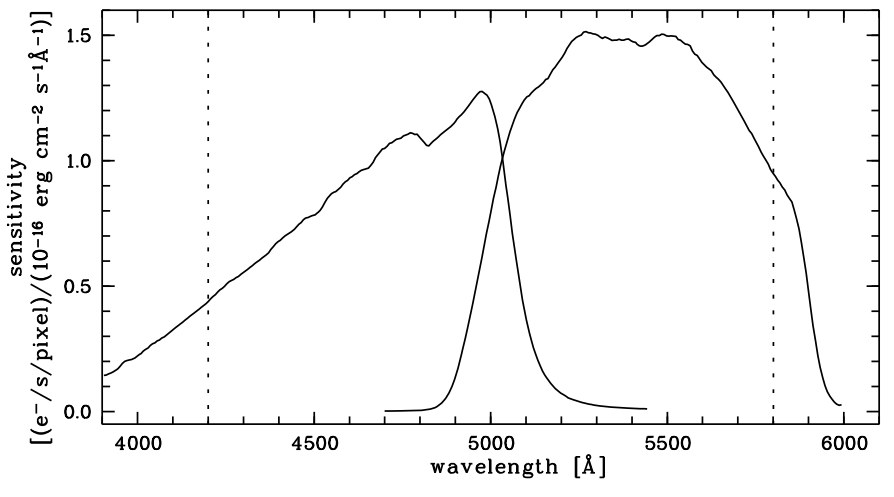

Fig. 4. Sensitivity curves of the WFI $B$ and $V$ filters derived from the spectrophotometric standard HD 49798. At $\sim 4700 \AA$ one triggered photoelectron per second per $\simeq 6.7 \AA$ A pixel corresponds to a flux density of $10^{-16} \mathrm{erg} \mathrm{cm}^{-2} \mathrm{~s}^{-1} \AA^{-1}$. Dotted lines mark the considered wavelength range.

10. Final spectra: the calibrated filtered spectra were spliced together in their overlapping spectral range. Spectra taken in both instrument rotations were reduced separately and then combined by weighting with their inverse variances. The final stack of slitless spectra from a field contained every source from the DSS input catalogue that had been recorded even in a single exposure due to dithering and field rotation.

Compared to standard imaging with the WFI, the inserted redblazed R50 grism reduces the instrument throughput by $\sim 15 \%$ and $\sim 36 \%$ in the $V$ and $B$ bands, respectively. The low sensitivity in the blue is to some extent balanced by a lower sky background compared to the $V$ band. After inspecting the extracted spectra of one field we defined the useful wavelength range to $4200 \AA \leq \lambda \leq 5800 \AA$. In each field we extracted 800-1600 meaningful spectra, the exact number varying with the depth of the input catalogue and with Galactic latitude. In total, slitless spectra of $\sim 29000$ objects were extracted in the 18 survey fields. The control field in the ECDFS contained an additional 1000 sources.

\subsection{Survey depth}

The magnitude limit of our survey obviously depends on both the depth of the input source catalogues and on the obtainable $S / N$ in the slitless spectroscopy data. We estimated the completeness of the source catalogues as a function of magnitude by considering the differential source counts. Figure 5 shows the 


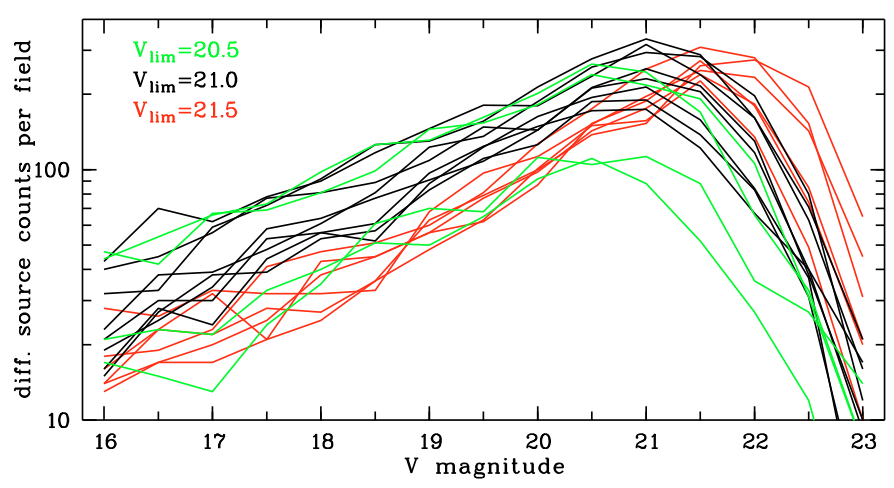

Fig. 5. Differential source density of the survey fields in $\Delta V=$ 0.5 mag bins derived from the DSS plates. The colours indicate different estimated limiting magnitudes $\left(V_{\lim } \simeq 20.5\right.$ : CTQ 0460, Q 0302-003, Q 1209+093, PKS 2126-158, $V_{\lim } \simeq 21.0$ : BR 1117-1329, BR 1202-0725, HE 0940-1050, HE 2243-6031, PKS 0528-250, Q 0420-388, Q 1451+123, Q 2139-4434, $V_{\lim } \simeq 21.5$ : CTQ 0247, Q 0055-269, HE 2347-4342, Q 0000-263, Q 0002-422, Q 0347-383).

differential surface densities per $\Delta V=0.5$ mag obtained from the DSS source catalogues in the 18 fields. From the breaks in the differential number counts we conclude that the input catalogues are reasonably complete to $V \sim 21$, with 4 fields being shallower by $\sim 0.5 \mathrm{mag}$. Similar limiting magnitudes were obtained in the three fields with direct WFI data.

The limiting magnitude of the slitless survey material was determined by evaluating the continuum $S / N$ of the extracted spectra. The $V$ magnitude of each object was calculated by integrating the flux over the $V$ band which is completely covered by the spectra. We estimate that the $V$ magnitudes of $V \lesssim 20 \mathrm{ob}-$ jects are accurate to $\sim 0.1 \mathrm{mag}$. Fainter sources have larger uncertainties due to their lower $S / N$. The $S / N$ is a function of the wavelength-dependent system sensitivity (Fig. 4) and of the source spectral energy distribution. We defined the median $S / N$ in the $V$ band as a characteristic value. Recall that the extraction yielded a noise array along with each spectrum, so that the $S / N$ can be estimated rather accurately. In Fig. 6 we plot the median $S / N$ of all extracted $\sim 1800$ spectra in one particular field, as a function of $V$ magnitude. One can see that a $S / N$ of 3 is obtained at $V \simeq 21.0$, and for $V \simeq 22.0$ a $S / N$ of unity is reached.

Some objects appear to have too low $S / N$ for their $V$ magnitude; these generally have shorter net exposure times induced by dithering and field rotation. After excluding these outliers we fitted a simple log-linear relation for the $S / N$ as a function of magnitude,

$V_{\mathrm{WFI}}=-2.5 \log (S / N)+22.05$

with a root-mean-square deviation between individual fields of $\sim 0.1 \mathrm{mag}$. The $S / N$ of very bright objects is systematically lower than this relation because of large non-Gaussian tails of the PSF which were not taken into account in the extraction, and because of detector saturation occurring at $V_{\text {WFI }} \lesssim 13.4$ (corresponding to $130000 \mathrm{e}^{-}$per pixel in $600 \mathrm{~s}$ ).

We conclude that at a magnitude of $V \simeq 21$, our survey should be reasonably complete in terms of the input catalogues, and that the slitless spectra at these magnitudes are generally still good enough to search for emission-line objects. We demonstrate below that the selection of even fainter $z>2.4$ quasars with prominent Ly $\alpha$ lines was also possible, in some fields down to $V \simeq 22$ or beyond (corresponding to a continuum $S / N$

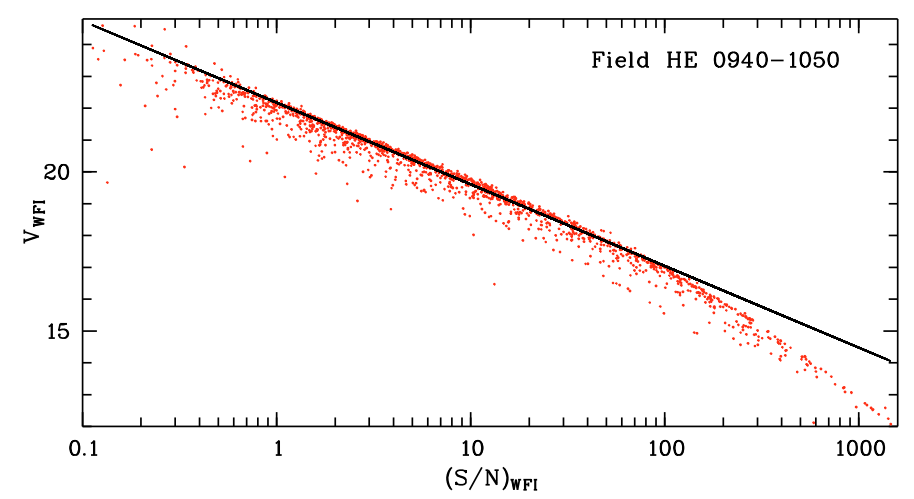

Fig. 6. Integrated WFI $V$ magnitude vs. median $S / N$ in the WFI spectra in the field of HE 0940-1050 (dots). The line shows the fitted log-linear relation of the magnitude and the $S / N$ for this field.

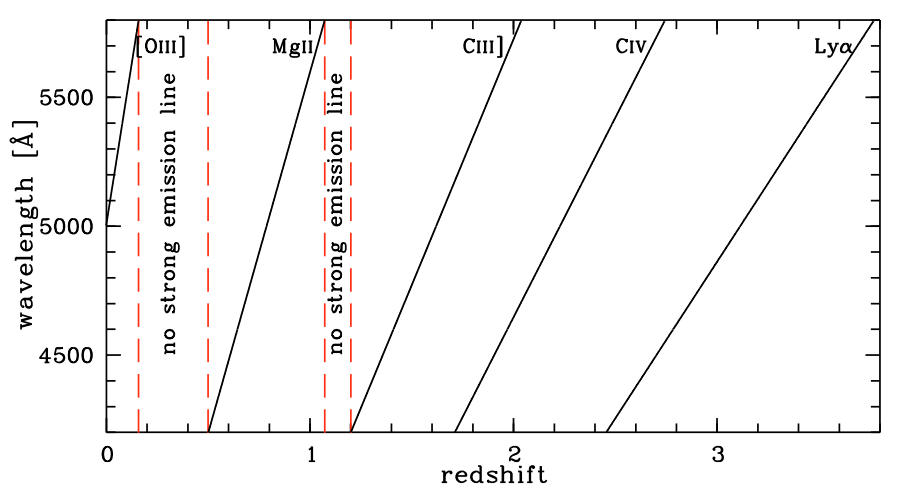

Fig. 7. Visibility of strong emission lines over the covered wavelength range. Dashed lines mark gaps without major emission lines where quasar candidates are missed.

of 1 in the slitless spectra). However, it is clear that only the most promising candidates will be selected at these faint levels. Moreover, we have seen that at $V \simeq 22$, already the input catalogues are heavily affected by approaching to the limits of the DSS itself. We return to a more quantitative assessment of (in)completeness of the quasar samples in Sect. 7.2 below.

\section{Selection of quasar candidates}

\subsection{Selection criteria}

Quasar candidates were semi-automatically selected from the calibrated slitless spectra on the basis of prominent emission lines. Figure 7 shows the redshift ranges in which major quasar emission lines can be observed in the slitless spectra. The detectability of the various emission lines depends on their equivalent widths and the $S / N$ in the data (Sect. 7.2). Moreover, quasar candidates could not be selected in two narrow low-redshift ranges due to the lack of major emission lines (see Fig. 7).

We first estimated a (pseudo-)continuum for each spectrum by applying a simple median filter with a window width of $400 \AA$. The continuum-subtracted spectra were searched automatically for emission line objects by cross-correlating them with Gaussian emission line templates. The fields around Q 0000-263, Q 0302-003 and Q 2139-4434 were chosen for testing the continuum subtraction and the line detection algorithm, ensuring that all emission line objects selected by eye were also found automatically. Finally, we chose two Gaussians with dispersions $\sigma_{\lambda}=40 \AA$ and of $\sigma_{\lambda}=70 \AA$ in order to match 
emission lines with small and large widths, respectively. Only features detected with a total line $S / N \geq 3$ and an observed equivalent width of $W_{\text {obs }} \geq 20 \AA$ were kept.

At our spectral resolution, the continuum subtracted spectra of normal stars often appeared to show "emission lines" that in reality were artefacts of poor continuum estimation and oversubtraction. The initial samples of emission line objects contained therefore $\sim 1 / 4$ of all extracted spectra. However, it was quite straightforward to beat down this number significantly. First of all, the equivalent widths of these features were generally quite small. Secondly, most of the spuriously selected stars had rather red colours. We quantified a colour measure by defining a "hardness ratio" 2 as

$H=\frac{f_{\lambda, V}-f_{\lambda, B}}{f_{\lambda, V}+f_{\lambda, B}}$

where $f_{\lambda, B}$ and $f_{\lambda, V}$ are the average fluxes in the intervals $4200 \AA \leq \lambda \leq 5000 \AA$ and $5000 \AA \leq \lambda \leq 5800 \AA$, respectively. Thus, the larger $H$, the redder the object.

We finally decided to treat emission line candidates with large equivalent widths differently from those with weak line detections. A strong-lined object was defined as one with a line $S / N \geq 3$ and $W_{\text {obs }} \geq 100 \AA$. If the line was detected in the $B$ band, the object needed to be moderately blue, $H-\sigma_{H} \leq$ 0.046; this criterion removed most of the red stars. If the line was detected in the $V$ band, no further restriction was applied. A line detection with small equivalent width, $20 \leq W_{\text {obs }}<100 \AA$, needed always to be accompanied by a blue but meaningful spectrum, given by the conditions $H+\sigma_{H}<0.15$ and $H-\sigma_{H}>-1$.

Essentially all Ly $\alpha$ detected quasars, but also some of the $\mathrm{C}$ IV and [O III] detections have large equivalent widths and therefore fell into the first set of criteria. Quasars at lower redshifts generally have weaker lines (C IV, C III], Mg II and [O III]), but are distinctly blue. Note that our highly conservative colour cuts were only applied after the emission line object selection; these cuts would by no means have sufficed to select quasar candidates on the basis of their colours.

After these cuts we visually inspected the selected objects to cull the quasar candidates from the remaining sample of slitless spectra, dominated by three types of spurious detections: (i) $\mathrm{M}$ stars with strong $\mathrm{TiO}$ absorption bands that our selection criteria did not catch; these features are always at the same wavelengths and could be recognised with good confidence after some training; (ii) low-redshift emission line galaxies with [O III] visible at $z \lesssim 0.15$; at the low resolution of the WFI spectra, the [O III] doublet is blended, resulting in an asymmetric single line that could be unambiguously identified in almost all cases (see Fig. 8, for examples). Since emission line galaxies are interesting in their own right, we kept them in a separate bin; (iii) defective spectra were overlapping 1st order spectra of two close objects, or spectra with unrecognised zero order contamination or spectra with unphysical breaks at $\sim 5000 \AA$ resulting from an imperfect splicing of the two bandpasses. Any remaining spectral overlaps were easily identified by comparing the spectra obtained at the two instrument rotation angles. All emission line candidates that passed the visual check of the one-dimensional spectra were finally verified on the slitless WFI images to check for any remaining flaws that could result in a spurious emission line. For objects at the field edges detected in only one instrument rotation (Fig. 1) this visual examination was essential. In total, 387 emission line objects were selected from the

\footnotetext{
${ }^{2}$ We borrowed this concept from the X-ray community because it is more reliable and less prone to noise peaks for very low $S / N$ data.
}

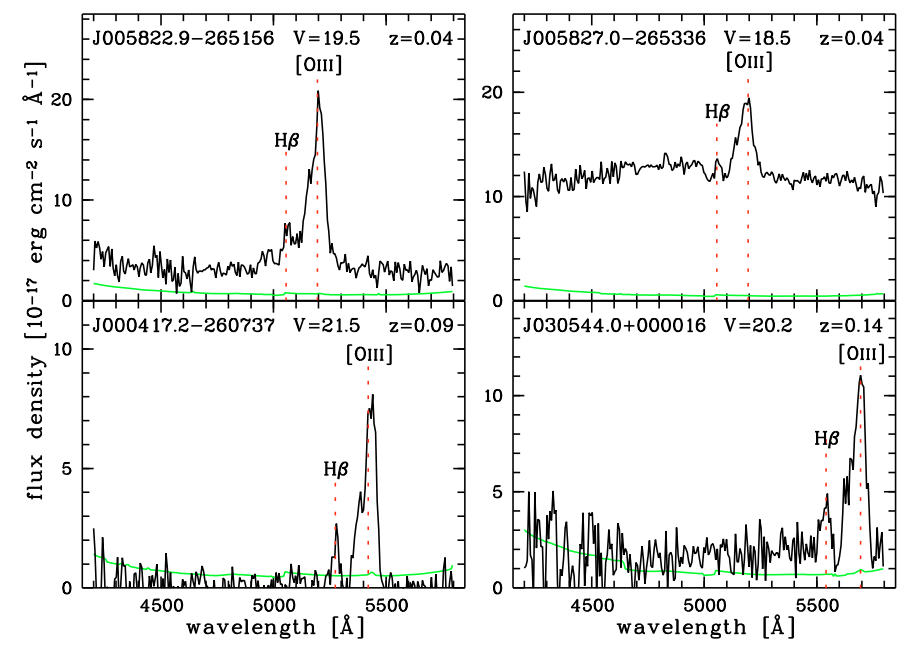

Fig. 8. Slitless spectra (black) and corresponding $1 \sigma$ noise arrays (green/grey) of four emission line galaxies showing blended [O III] emission. $\mathrm{H} \beta$ can be recognised as well.

$\sim 29000$ objects detected on the DSS images of the 18 survey fields.

Visual inspection of the sky-subtracted slitless images revealed three additional candidates that were missed in the DSS catalogues, mainly due to source blending. These fields were reprocessed with the source catalogues of available direct WFI data. At the higher resolution of the direct WFI data, these candidates were resolved and their spectra were successfully extracted. Figure 9 presents the slitless $B$ band data and the corresponding direct WFI image of a region near HE 0940-1050 where two prime quasar candidates were discovered. On the DSS image of this field, QNQ J09434-1053 could not be resolved from the nearby foreground object. The WFI catalogues did not reveal further undetected candidates, confirming our expectation that the fraction of candidates lost due to blended sources is very small.

\subsection{The sample of quasar candidates}

In total, our semi-automated search for emission line objects yielded 390 sources on the surveyed $4.39 \mathrm{deg}^{2}$. Of those, 38 we rejected as dubious due to very low $S / N$. The remaining sample of 351 objects consists of 37 known quasars (including 15 of the 18 targeted central quasars), 169 quasar candidates, and 145 candidate low-redshift star-forming galaxies. The classification was purely based on the slitless spectra. No morphological selection was made.

Most of the emission line galaxies were identified by their blended [O III] doublet. However, due to low $S / N$ at this low resolution $R \sim 50$, we could not determine whether the $\mathrm{H} \beta$ lines seen in the spectra are broad or narrow, so that this sample of low-redshift objects might still contain low-redshift AGN. At the bright end of our survey ( $V \lesssim 18$ ), the surface density of $z \lesssim$ 0.15 emission line galaxies is $\sim 1.1 \mathrm{deg}^{-2}$, which compares well with other slitless surveys (Salzer et al. 2002; Bongiovanni et al. 2005). As most of the deeper surveys for emission line galaxies select $\mathrm{H} \alpha$ emission (e.g. Pascual et al. 2001), a comparison at fainter limits is difficult.

In total, we rediscovered 37 previously known quasars in the survey fields with detectable emission lines in the observed wavelength range. Three of the central quasars (Q 0000-263, BR 1117-1329 and BR 1202-0725) reside at too high redshift 

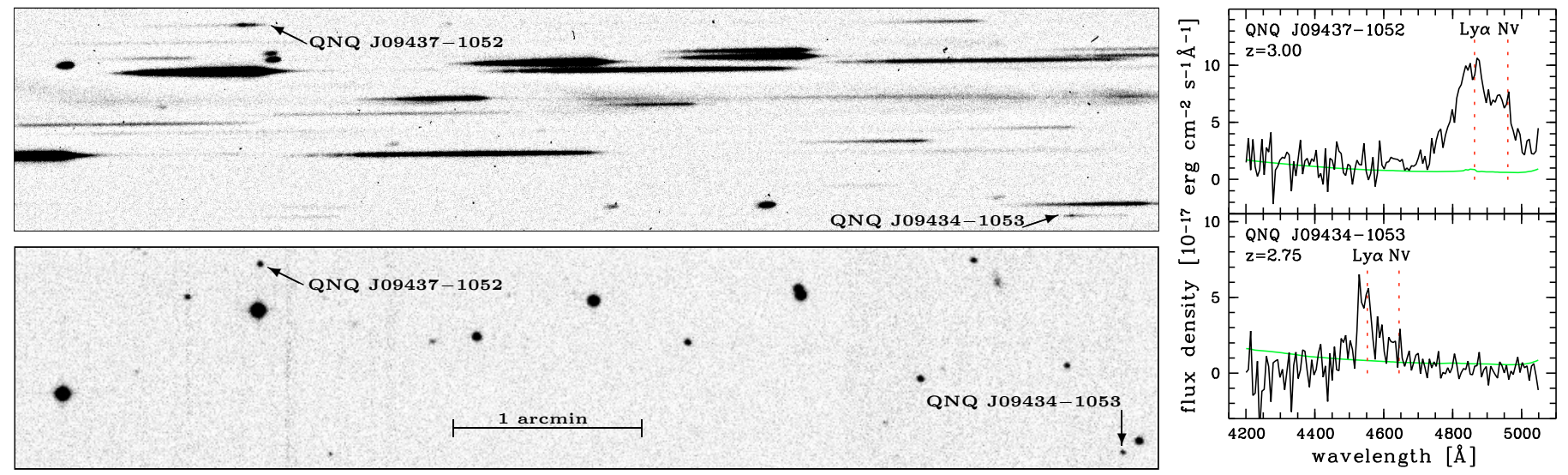

Fig. 9. Slitless WFI spectra of two newly discovered quasars near HE 0940-1050. The upper left panel shows a close-up of the combined slitless $B$-filtered exposures in the field of HE 0940-1050 taken without rotation (Fig. 2). The dispersion runs horizontally from left to right. The lower left panel shows a direct WFI $B$ band image of the corresponding sky region north-east of HE $0940-1050\left(6{ }^{\prime} 10 \times 11^{\prime} 19\right)$. The indicated objects display prominent emission lines in the slitless data. The right panels show the automatically reduced and calibrated one-dimensional $B$ band spectra of the two quasars (black) and their $1 \sigma$ noise arrays (green/grey). Both of them display Ly $\alpha$ emission blended with $\mathrm{N} V$ at the indicated redshifts.

to be detected in emission. Excluding the central bright quasars, 10 out of 12 known quasars are rediscovered in the range of high selection probability $(V \lesssim 21,1.7<z<3.6$, see Sect. 7.2 below). One of the two missed quasars had been recorded in one WFI rotation only, whereas the other one was not contained in the shallow DSS source catalogue of the Q 0302-003 field (Fig. 5). The redshifts of the rediscovered quasars measured in the slitless data are consistent (within $\sigma_{z} \simeq 0.03$ ) with the published values (see Sect. 6.2 and Fig. 11 below).

We estimated redshifts of the 169 quasar candidates from the slitless data, and the candidates were grouped by redshift confidence. The redshifts were considered to be almost secure if either two emission lines with a consistent redshift were present in the spectral range, or if a single line could be confidently identified due to its shape and/or that of the adjacent continuum. In particular, Ly $\alpha$ emission blended with $\mathrm{N} \mathrm{V}$ could often be unambiguously identified (for examples see Fig. 9). Bright low-redshift candidates displayed lines with small equivalent widths (likely $\mathrm{C} \mathrm{III]} \mathrm{or} \mathrm{Mg}$ II) superimposed on a blue continuum. Redshift assignments of fainter quasar candidates were less certain due to the similar equivalent widths of the C IV, C III] and $\mathrm{Mg}$ II emission lines. We decided in all doubtful cases to assign the highest plausible redshifts to the candidates.

For eight of our fields we retrieved WFI+grism $R$ band data from the ESO Science Archive (taken in the course of another programme, PI Vanzi). We reduced the data with our pipeline, yielding calibrated $R$ band spectra of our candidates in the range $5800 \AA \leq \lambda \leq 7400 \AA$. Due to the much higher sky background, however, the $R$ band spectra are considerably noisier than the $B$ and $V$ band spectra. Therefore, only emission lines of brighter objects of our sample were unambiguously detected. Nevertheless, the $R$ band spectra confirmed the previously adopted low redshift assignments of 8 candidates. No systematic search for further emission line objects was performed among the $R$ band spectra.

\section{Spectroscopic follow-up}

\subsection{Observations}

Follow-up spectroscopy of 81 quasar candidates was obtained with the Focal Reducer/Low Dispersion Spectrograph 2 (FORS2, Appenzeller et al. 1998) on ESO VLT UT1/Antu in
Visitor Mode on November 17-19, 2004 and in Service Mode between April and July 2005. We restricted the follow-up campaign to candidates with a high-confidence slitless WFI redshift $z \geq 1.7$. Quasar candidates likely residing at lower redshifts (C III] or Mg II seen in the slitless spectra) were not followed up. The field of PKS 0528-250 was not selected for follow-up and also the field of Q 1451+123 was not included due to the lack of high-quality candidates. Longslit spectra with a $1^{\prime \prime}$ slit kept at the parallactic angle were taken either with the $300 \mathrm{~V}$ grism or the 600B grism, resulting in a spectral resolution of $\sim 10 \AA$ FWHM and $\sim 4.5 \AA$ FWHM, respectively. Each resolution element is oversampled by a factor of $\sim 3(300 \mathrm{~V}: 3.36 \AA /$ pixel, 600B: $1.5 \AA /$ pixel). No order separation filter was employed to maximise the UV coverage in the spectra, leading to possible order overlap at $\lambda>6600 \AA$ in the spectra taken with the $300 \mathrm{~V}$ grism. Exposure times were adjusted to yield $S / N \sim 20$ in the quasar continuum and ranged between one and $40 \mathrm{~min}$. The sky was clear to photometric during the Visitor Mode run, but the seeing varied strongly during the nights, resulting in slit losses for some of the 53 targeted objects. The remaining 28 prime quasar candidates were observed in Service Mode under variable conditions. The spectra were calibrated in wavelength against the FORS2 $\mathrm{He} / \mathrm{Ne} / \mathrm{Ar} / \mathrm{HgCd}$ arc lamps and flux-calibrated via spectrophotometric standard stars taken each night. However, absolute spectrophotometry was not achieved due to the narrow slit and the sometimes variable or poor sky transparency in the Service Mode observations. Table 2, available in the online edition of the Journal, lists the quasars confirmed in the spectroscopic follow-up observations.

\subsection{Data reduction}

The VLT spectra were reduced with standard $\mathrm{IRAF}^{3}$ tasks. The bias value was taken from the overscan regions. Dome flatfields were used to correct pixel sensitivity variations. After sky subtraction by fitting both spatial regions close to the target by 2nd-order Chebychev polynomials, the spectra were extracted

3 IRAF is distributed by the National Optical Astronomy
Observatories, which are operated by the Association of Universities
for Research in Astronomy, Inc., under cooperative agreement with the National Science Foundation. 


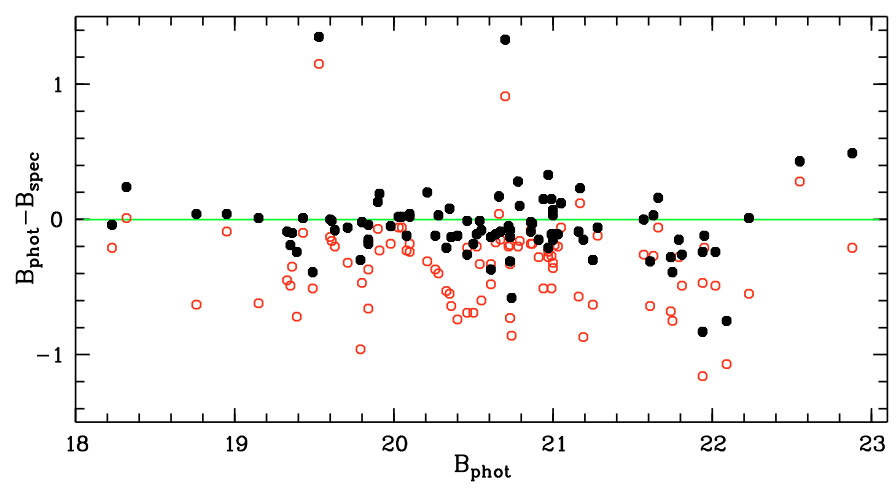

Fig. 10. Blue magnitudes of the quasar candidates observed with the VLT. The abscissa shows the photometric $B$ magnitudes obtained from the aquisition images, the ordinate the difference to the magnitudes integrated from the slit spectra. Full (open) circles correspond to measurements with (without) correction for slit losses. The line marks a perfect 1:1 relation between $B_{\text {phot }}$ and $B_{\text {spec }}$.

with the optimal extraction algorithm by Horne (1986). The root-mean-square residuals of the wavelength calibration with low-order Chebychev polynomials were $\lesssim 0.05 \AA$ and $\sim 0.2 \AA$ for the $600 \mathrm{~B}$ and the $300 \mathrm{~V}$ grism, respectively. The spectra were corrected for atmospheric extinction assuming average conditions.

In order to approximately correct the spectra for slit losses we carried out aperture photometry on the FORS2 aquisition images taken in the $B$ filter, which were calibrated against photometric standard stars (Landolt 1992) taken each night. Table 2 provides the airmass-corrected Johnson $B$ magnitudes and their calculated standard deviations. Apparent magnitudes were also derived by integrating the flux-calibrated slit spectra. Slit losses were estimated by calculating the expected loss over the used 1 " slit for a Gaussian PSF with a FWHM equal to the seeing during the observations that we measured on the aquisition images. Figure 10 presents the correlation of the integrated and photometric magnitudes. The integrated magnitudes are consistent with the photometric ones after correcting for slit losses. The two outliers are the two quasars QNQ J09425-1048 and QNQ J21294-1521 that were observed in strongly variable clearly non-photometric conditions.

\section{Results}

\subsection{Redshift determination}

Redshifts of all candidates observed with FORS2 were determined by taking into account all detectable emission lines. Peak positions of the lines were measured by eye and errors were estimated by considering the $S / N$ of the lines, blending with other emission lines and the presence of absorption lines, sky residuals or line asymmetries. We confirmed that in many objects highionisation lines (Ly $\alpha, \mathrm{N}$ V, Si IV+O IV], C IV) are blueshifted with respect to low-ionisation lines (Gaskell 1982; Tytler \& Fan 1992; McIntosh et al. 1999; Richards et al. 2002b). However, the low-ionisation lines O I+Si II and C II were often weak and noisy, resulting in larger individual redshift errors. $\mathrm{Mg}$ II could be measured only for quasars at $z \lesssim 2.30$ ( $z \lesssim 1.25$ ) taken with the $300 \mathrm{~V}(600 \mathrm{~B})$ grism. Therefore, we estimated the systemic redshift of each quasar by weighting the measurements of individual lines, giving a lower weight to high-ionisation lines or discarding them completely in case of large blueshifts. The redshift uncertainty of each quasar was estimated from the redshift

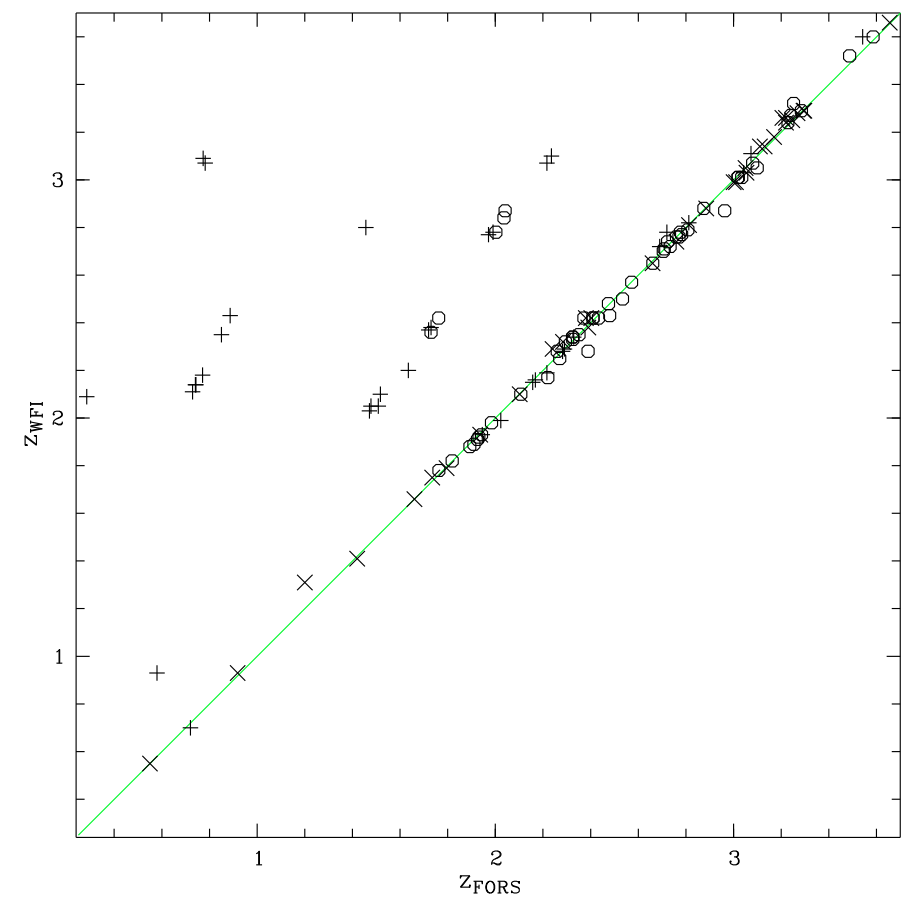

Fig. 11. Comparison of the redshifts obtained with FORS2 and WFI. Circles (+ signs) denote quasar candidates with almost secure (uncertain) WFI redshifts. Previously known quasars in the fields have been added with their published redshift in comparison to the redshift measured in the slitless spectra $(\times$ signs $)$. The line marks a 1:1 relation.

scatter between the remaining lines and their centroiding errors. The adopted quasar redshifts and their estimated errors are listed in Table 2 .

\subsection{Newly confirmed quasars}

With the follow-up spectroscopy we confirmed that 80 of our 81 candidates are broad-line AGN in the redshift range $0.580 \leq z \leq 3.586$. Only one of the candidates turned out to be a low-redshift galaxy with narrow emission lines (object J03490-3820, $\alpha(\mathrm{J} 2000)=03^{\mathrm{h}} 49^{\mathrm{m}} 00^{\mathrm{s}} .56$, $\left.\delta(\mathrm{J} 2000)=-38^{\circ} 20^{\prime} 31^{\prime \prime} .9, z=0.2848\right) .12$ of the confirmed quasars, located in the fields around Q 0302-003 and HE 2347-4342, were already presented and discussed in Papers I and II. In Fig. 12 we show the spectra for the quasars in one additional, particularly rich field. 11 quasars were found in the vicinity of HE 0940-1050. Appendix A, available in the online edition of the Journal, lists the spectra of the remaining 57 newly discovered quasars.

In Fig. 11 we compare the redshifts derived from the FORS2 spectra and the slitless WFI spectra, respectively. The slit spectra confirmed the redshifts determined from the slitless WFI spectra for the majority of candidates (54/81). The WFI redshifts of the other 27 objects in the follow-up had been overestimated (note that we had systematically assigned the highest plausible redshifts). 64 of the 80 confirmed quasars are above our low-redshift cutoff $z=1.7$ set for inclusion in the follow-up.

The scatter between slit and slitless spectroscopic redshifts for the 54 quasars correctly placed in $z$ is $\sigma_{z} \simeq 0.03$. The main cause for this scatter is optical distortions of the grism causing the astrometric transformation to produce residual variations of the wavelength zeropoint across the WFI field of view. Of the 27 candidates whose redshifts were overpredicted, only 5 had 

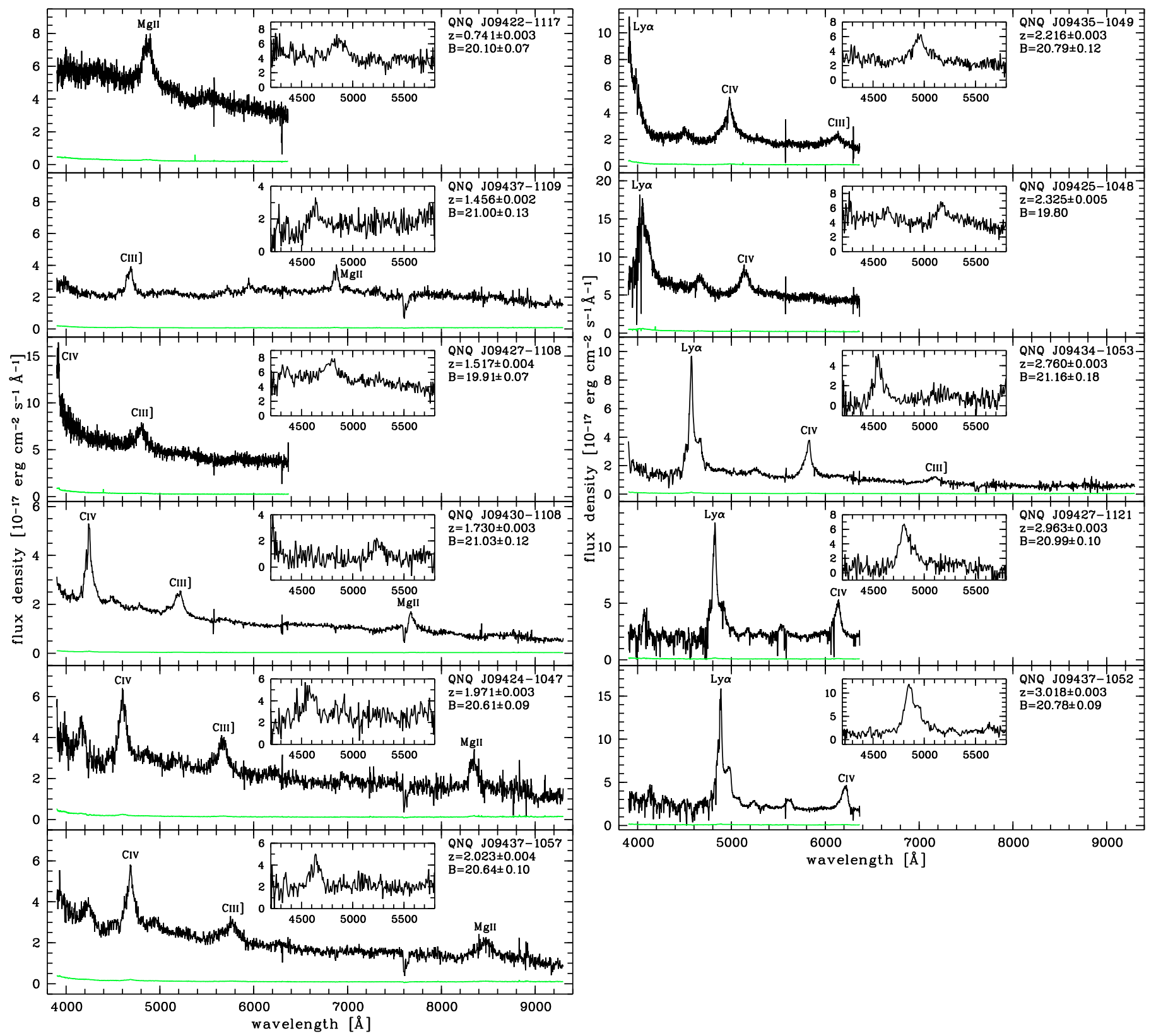

Fig. 12. VLT/FORS2 spectra of the 11 discovered quasars in the vicinity of HE 0940-1050. The spectra are shown in black together with their $1 \sigma$ noise arrays (green/grey). The spectra have been scaled to yield the measured photometric $B$ magnitudes. Major emission lines are labelled. The small inserts show the corresponding slitless WFI spectra in the same units.

been assigned a high confidence redshift. This suggests that the majority of remaining candidates with high redshift confidence likely reside at those redshifts. After completion of the followup observations we compared the WFI spectra of quasars whose redshifts had been overestimated to the WFI spectra of the remaining candidates and adjusted the redshift estimates where applicable.

\subsection{Quasar candidates not included in the follow-up}

With 169 candidates in our sample and 81 of them followed up by VLT slit spectroscopy, 88 further possible quasars remain unconfirmed. For the benefit of potential future users we list these in Appendix B, available in the online edition of the Journal. We provide the slitless spectra of these 88 quasar candidates and their celestial coordinates, magnitudes, WFI redshifts, and our assessment of redshift confidence. For 23 of these we judge the WFI redshifts to be most likely correct. For 28 candidates we call our redshift estimate plausible; for 37 they are uncertain and could be identified with any of the major emission lines, although we hold it unlikely that many Ly $\alpha$ quasars will be hidden among them. As an example we show in Fig. 13 the slitless spectra of 10 quasar candidates in the field of PKS 0528-250 that was completely left out of the spectroscopic follow-up. Three of these candidates have a secure redshift $z>2.5$. The remaining candidates on the other fields are either faint with noisy WFI spectra or they likely reside at redshifts $z<1.7$.

\section{Discussion}

We summarise the overall redshift distribution of the quasar sample in Fig. 14. The open histogram shows the redshift 

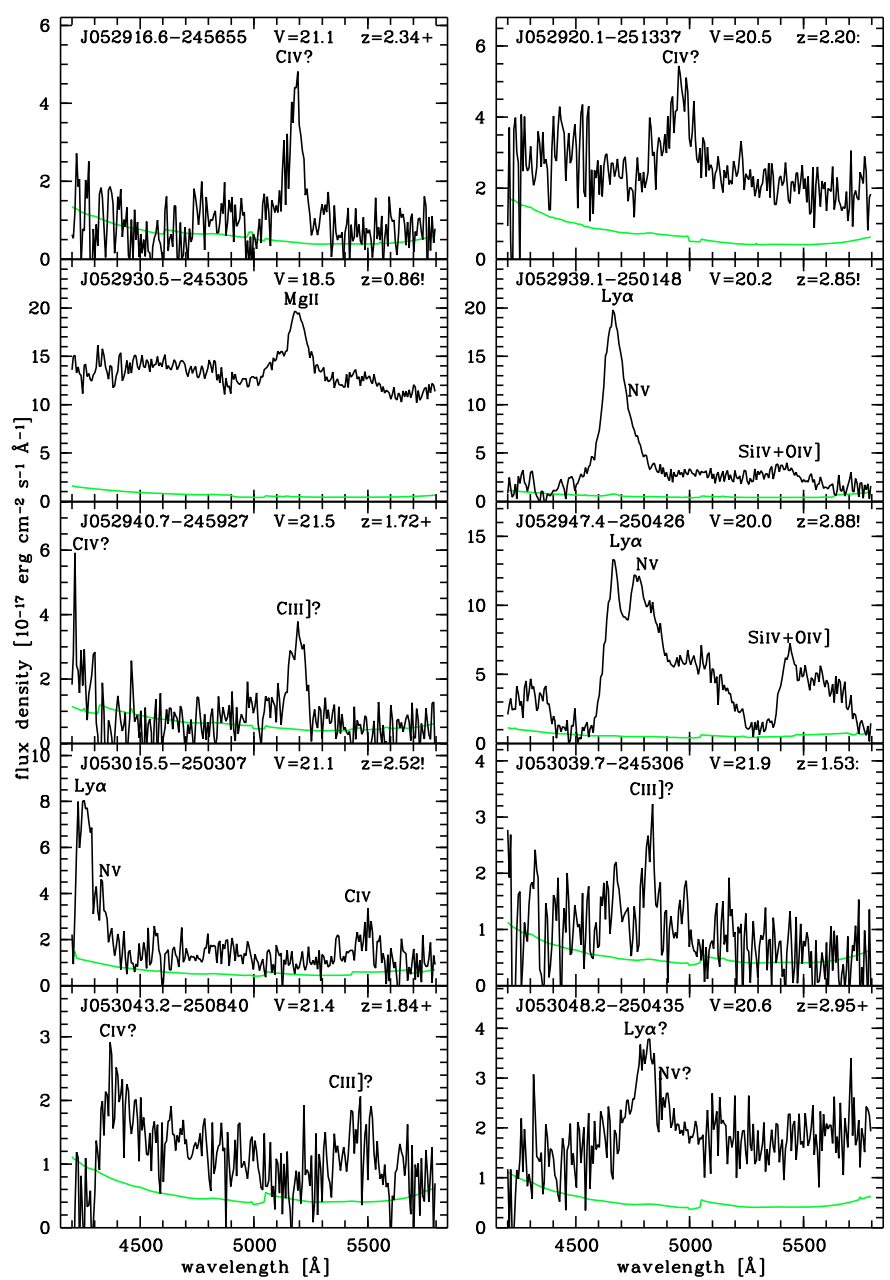

Fig. 13. Slitless spectra (black) and corresponding $1 \sigma$ noise arrays (green/grey) of 10 quasar candidates in the field of PKS 0528-250 with indicated coordinates, approximate $V$ magnitudes and estimated redshifts with confidence level $(!=$ secure,$+=$ plausible, $:=$ uncertain $)$. Major emission lines are labelled.

distribution of all detected known quasars, newly discovered quasars and remaining candidates assuming that the inferred WFI redshifts of the candidates are correct. In total, 205 quasars and candidates are shown. The gap at $1 \lesssim z \lesssim 1.3$ expectedly arises from the lack of visible quasar emission lines in the observed spectral range (cf. Fig. 7). The very small number of lowredshift $(z \lesssim 0.6)$ AGN is also to this effect. For $z \gtrsim 1.4$, no clearly preferred redshifts are discernible from this histogram. In Sect. 7.2 we present an attempt to quantify our redshift- and magnitude selection function. First we discuss our results in terms of the main rationale driving this survey, the construction of new apparent groups of high-redshift quasars.

\subsection{New quasar groups}

The main goal of this survey was to reveal new groups of quasars for further studies of the three-dimensional distribution of the intergalactic medium. In the follow-up observations we concentrated on likely high-redshift $(z>1.7)$ quasars, with some success: $80 \%$ of the newly established quasars match that condition. In addition to the central quasars, we recovered $85 \%$ of all detectable previously known quasars in these fields (22/26).

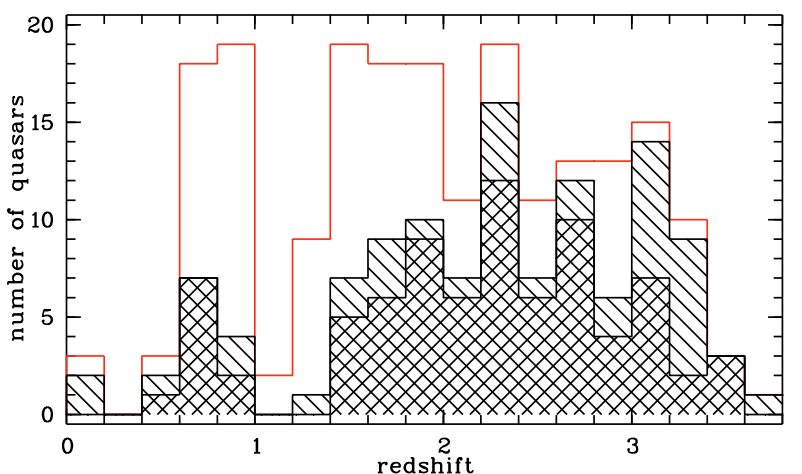

Fig. 14. Redshift histograms of confirmed previously unknown quasars (cross-hashed), rediscovered quasars (hashed) and remaining quasar candidates (open).

Including the remaining candidates, the number of $z \geq$ 1.7 quasars with $V \lesssim 21$ per 26 '. $2 \times 33$ '. 5 field varies substantially, between 2 (near CTQ 0247, Q 1209+093 and Q 1451+123) and 11 (near Q 0347-383). Some of our fields are thus almost devoid of high-redshift quasars except the central object, but other fields we found to be quite richly populated. These fields may in the future become interesting targets for multiple line of sight absorption spectroscopy. A list of currently known $z \geq 1.7$ quasars in our survey fields is provided in Table C. 1, available in the online edition of the Journal. We briefly discuss some of the most prominent cases:

- HE 0940-1050 $(z=3.088, V=16.4)$ : the high-quality VLT/UVES and Keck/HIRES spectra of HE 0940-1050 have been heavily used in recent quasar absorption line studies, for example to constrain the evolution of the fine structure constant (Murphy et al. 2003; Chand et al. 2004) and to obtain precise measurements of the flux distribution in the Ly $\alpha$ forest (Becker et al. 2007; Kim et al. 2007). So far, no other quasars or AGN have been known within a radius $<30^{\prime}$ around HE 0940-1050. We confirmed 11 out of 19 quasar candidates. In the upper panels of Fig. 15 we plot the angular distribution of quasars and quasar candidates with respect to HE 0940-1050 together with the distribution of the quasars on the sky. Six confirmed foreground quasars of HE 0940-1050 are located at $z>2$. The newly established quasars in this field form two close subgroups on the sky with separations of $1 ! 8<\vartheta<8$. 8 . Thus, this field is well suited to study transverse correlations in the IGM on small scales towards the newly discovered quasars in addition to the larger scales given by their separations to the central quasar.

- $Q 0302-003(z=3.285, V=17.7)$ and $H E$ 2347-4342 $(z=2.885, V=16.4)$ : these two quasars are among the very few for which intergalactic He II absorption has been detected by UV spectroscopy (e.g. Jakobsen et al. 1994; Reimers et al. 1997; Heap et al. 2000; Smette et al. 2002; Zheng et al. 2004). The association between the hardness of the intergalactic radiation field (inferred from H I and He II Ly $\alpha$ spectroscopy) and the presence of foreground quasars near the line of sight has been explored in Papers I and II, revealing for the first time the "transverse proximity effect in spectral hardness". In Paper II we also noted the remarkable apparent group of 6 quasars with $z>2.2$ that is less than $17^{\prime}$ from the central object, 3 of which are even behind HE 2347-4342.

- $Q$ 0000-263 $(z=4.125, R=17.5)$ : a $3.69 \times 5 ! 13$ region around this luminous high-redshift quasar was included in the Lyman break galaxy survey by Steidel et al. (2003), 

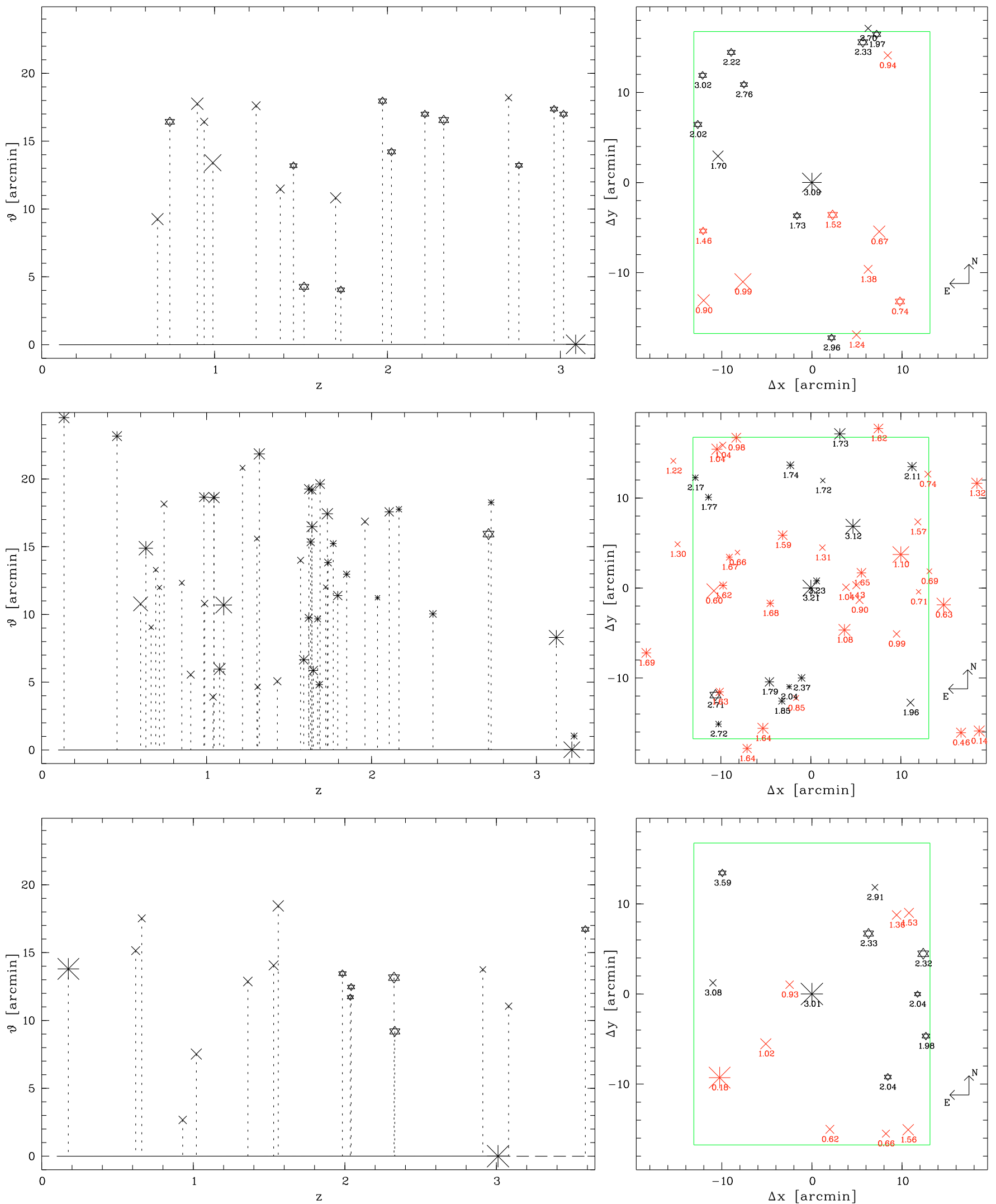

Fig. 15. Distributions of quasars in three of the survey fields (upper panels: HE 0940-1050, middle panels: Q 2139-4434, lower panels: HE 2243-6031). Left panels: angular separation distribution of quasars and quasar candidates with respect to the central quasar as a function of redshift. Asterisks and star symbols mark known quasars and newly discovered confirmed quasars, respectively. Crosses show remaining WFI quasar candidates. Symbol size indicates apparent optical magnitude. Right panels: quasar distribution on the sky centred on the targeted known quasar in the field with indicated redshifts. Objects at $z \geq 1.7(z<1.7)$ are shown in black (red/grey). The rectangle marks the nominal contiguous slitless WFI field of view $\left(26 ! 2 \times 33{ }^{\prime} 5\right)$ without rotating the instrument, for simplicity centred on the central quasar. New quasars located slightly outside the nominal field of view are due to field rotation and small offsets of the actual field centre. 
yielding also $3 g>24 \mathrm{AGN}$ at $z>3$. While these are obviously too faint to be rediscovered, our survey revealed 4 quasars at $2.77<z<3.07$ at larger separations to the central quasar.

- $Q 0347-383(z=3.220, V=17.7)$ : among the 10 confirmed quasars around Q 0347-383 there is a dense group of 4 quasars at $2.70<z<2.78$ with magnitudes $20.5<$ $B<21.0$. Thus, these quasars are well suited for mediumresolution spectroscopy of the Ly $\alpha$ forest on closely spaced sightlines. Two further $B \simeq 20.9$ quasars at $z=2.433$ and $z=$ 2.475 are located within a radius of $5^{\prime}$ around Q 0347-383.

- Q 0420-388 ( $z=3.120, V=16.9)$ : taken in the ESO Large Programme "The Cosmic Evolution of the Intergalactic Medium" (Bergeron et al. 2004), the UVES spectrum of Q $0420-388$ is one of the highest-quality quasar spectra taken so $\operatorname{far}(S / N \simeq 100$ per $0.05 \AA$ pixel). Five out of seven newly established foreground quasars near Q 0420-388 are located at $z>2$. The known quasar Q 0420-3850 $(z=2.410$, Hewitt $\&$ Burbidge 1993) separated from Q 0420-388 by $\vartheta=2 !$. was readily rediscovered in our survey. In total, this field now contains seven $z>2$ quasars and two other candidates with estimated high redshifts.

- Q 2139-4434 ( $z=3.214, V=17.70)$ : the sky region near this quasar (middle panels of Fig. 15) has been intensely studied in previous surveys. Morris et al. (1991) discovered both the central quasar Q 2139-4434 and another nearby bright highredshift quasar (Q 2138-4427, $z=3.120, \vartheta=8$ '.3). Deeper surveys revealed several fainter quasars (Véron \& Hawkins 1995; Hawkins 2000). D'Odorico et al. (2002) used highresolution spectra of the triplet formed by Q 2139-4434, its nearby companion Q 2139-4433 $\left(z=3.228, \vartheta=1^{\prime}\right)$ and Q 2138-4427 in order to reveal strong clustering of highcolumn density systems in the IGM in the plane of the sky. More recently, Francis et al. (2004) presented follow-up spectroscopy of colour-selected quasar and Ly $\alpha$ emitter candidates near Q 2139-4434, finding an overdensity of $z \simeq 1.65$ quasars and a large-scale filament of $\operatorname{Ly} \alpha$ emitting galaxies at $z=$ 2.38. Besides recovering the majority of the known quasars in this field, we identified a further quite bright high-redshift quasar (QNQ J21434-4432, $B=19.3, z=2.709$ ). Francis et al. (2004) discovered two fainter quasars at approximately the same redshift $(z=2.725)$. One of them is located just $3 ! 2$ south of QNQ J21434-4432. In total, there are now 14 confirmed $z>1.7$ quasars within a radius $<20^{\prime}$ around Q 2139-4434.

- HE 2243-6031 $(z=3.010, V=16.4)$ : the quasar distribution around HE 2243-6031 is shown in the lowest panels of Fig. 15. This group is of particular interest for further studies of quasar-absorber clustering. Near HE 2243-6031 our survey revealed two bright quasars (QNQ J22463-6009 and QNQ J22454-6011 from Table 2) that coincide in redshift with the $z=2.33$ damped Ly $\alpha$ absorber towards HE 2243-6031 discussed by Lopez et al. (2002). The foreground quasars are separated from the central line of sight by 9.2 and $13: 2$, corresponding to transverse comoving distances of 15.1 and $21.6 \mathrm{Mpc}$ in a flat universe with density parameters $\left(\Omega_{\mathrm{m}}, \Omega_{\Lambda}\right)=(0.3,0.7)$ and a Hubble constant $H_{0}=70 \mathrm{~km} \mathrm{~s}^{-1} \mathrm{Mpc}^{-1}$, respectively. Besides, this field contains another probably associated quasar pair at $z \simeq 2.04$. Moreover, the background quasar QNQ J22484-6002 ( $z=$ $3.586, V=19.3$ ) is well suited for high-resolution followup spectroscopy. This underlines the potential of our present quasar sample and the need for further investigations.
For the remaining fields plots corresponding to Fig. 15 are presented in Appendix $C$, available in the online edition of the Journal.

\subsection{Quantification of selection effects}

We have shown above that quasars displaying strong emission lines can be selected from the slitless spectra with very high efficiency; only 1 out of 81 candidates followed up with the VLT turned out not to be a quasar. Although in all parts of the survey we optimised the procedure towards efficiency, also at the expense of completeness, nevertheless it was interesting to see how our emission-line based candidate selection performed with respect to the quasar population displaying broad emission lines. We address this issue in two ways: first we present an estimate of the survey selection function from Monte Carlo simulations. Then we use this selection function to determine quasar surface densities from our sample and compare these numbers with the results of other surveys.

\subsubsection{Survey selection function}

We define the survey selection function as the recovered fraction of the broad-line quasar population matching a given broad band flux limit. The main variables governing the detectability of quasars in a survey such as ours are (i) the continuum magnitudes; and (ii) the equivalent widths of the detectable emission lines (Schmidt et al. 1986; Gratton \& Osmer 1987). Quasar surveys based on emission lines tend to preferentially select stronglined quasars, even more so near their survey limits. In order to quantify this selection effect, we first generated several template quasar spectra via a Monte Carlo routine. The quasar emission lines of Ly $\alpha$, Si IV+O IV], C IV, C III] and Mg II were modelled as Gaussian profiles with mean equivalent widths and line dispersions from the quasar template spectrum by Vanden Berk et al. (2001) superposed on a power law continuum $f_{v} \propto v^{-\alpha}$ with a mean spectral index $\alpha=0.5$. We incorporated variations in the model parameters by assuming Gaussian distributions with a standard deviation of $20 \%$ around the mean. We then generated 200 random spectral templates in the quasar rest frame. The neglect of non-Gaussian line shapes and intrinsic or intervening absorption does not have a big effect on our low-resolution spectra.

Mock slitless WFI quasar spectra were created by shifting the templates to the desired redshift, degrading them to the WFI resolution followed by adding Gaussian noise that varied with wavelength according to the overall throughput of the spectrograph (Fig. 4). The $S / N$ in the simulated spectra, related to the $V$ magnitude by Eq. (1), was normalised in the quasar continuum near the maximum throughput at $5400 \AA$. We then performed Monte Carlo simulations of the detection rates of quasars as a function of redshift $(0.6 \leq z \leq 3.7)$ and continuum $S / N$ values $(0.5 \leq S / N \leq 50)$. At each considered redshift and $S / N$, WFI spectra of the 200 quasar templates were generated and subjected to our automated selection routines (Sect. 4.1). Each quasar template was simulated 100 times in order to quantify the impact of noise on the detection rate per object. We also performed the subsequent visual screening done for the real candidates on several hundred simulated spectra to investigate visual selection effects, finding that $\gtrsim 90 \%$ of the automatically selected candidates survived the selection by eye.

Figure 16 presents the resulting selection function, displayed as a contour map of the median detection probability as a 


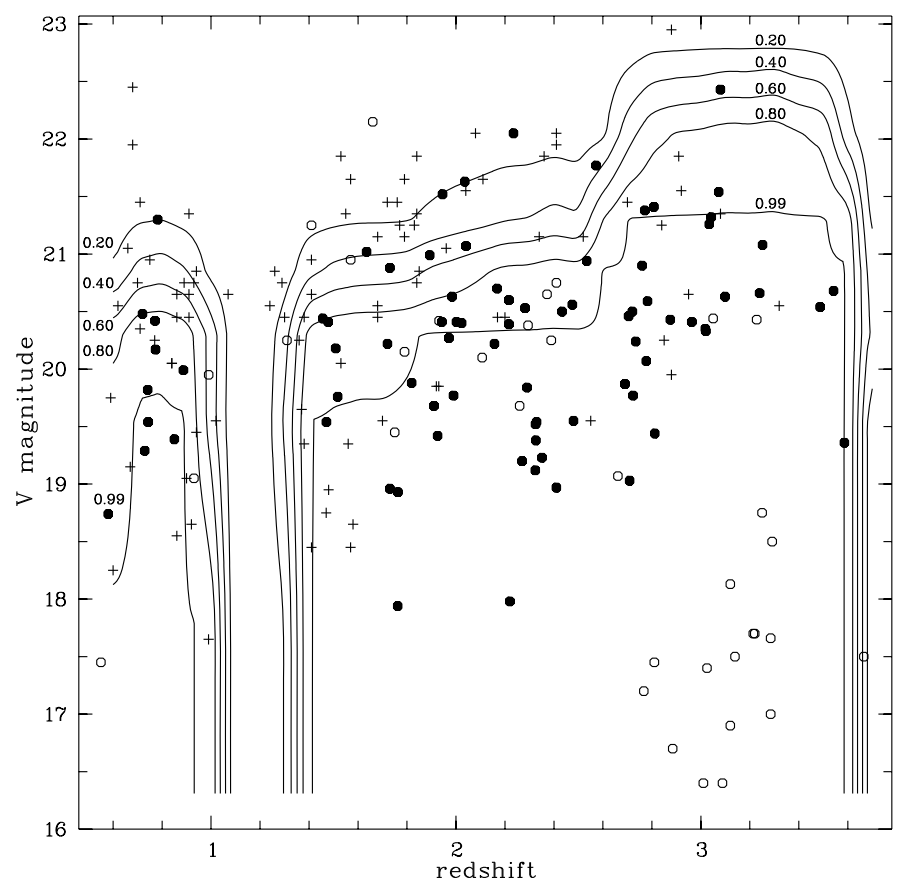

Fig. 16. Survey selection function as a function of redshift and apparent $V$ magnitude. Contours correspond to median simulated detection probabilities $p \in\{0.2,0.4,0.6,0.8,0.99\}$. Full and open circles mark newly discovered confirmed quasars and known quasars, respectively. Crosses denote remaining candidates.

function of redshift and $V$ magnitude. Overplotted are the quasars and quasar candidates from our survey. The selection function obviously varies with the visibility of different emission lines in the spectrum. Due to its high equivalent width Ly $\alpha$ emission is detectable for essentially $100 \%$ of quasars brighter than $V \simeq 21$, and still $\sim 80 \%$ of the simulated spectra are recovered at $V \sim 22$ (continuum $S / N$ of $\sim 1$ ). At redshifts below $\sim 2.5$ the selection function obtains lower values because of the lower equivalent widths of C IV, C III], and Mg II, and we have already commented upon the gap at $1.05 \lesssim z \lesssim 1.30$ where no emission line is visible in the range of our spectra. The selected quasars and quasar candidates fill the full range of the selection function spanned by the simulated redshifts and magnitudes $(S / N)$. The known quasars at the field centres are clearly separated from the other quasars and candidates in magnitude and redshift. Note, however, that the survey selection function in Fig. 16 assumes that all quasar spectra were extracted from the slitless data. Due to photometric incompleteness (Sect. 3.2), the detection probability at $V \gtrsim 21$ is lower, as we will describe in the following.

\subsubsection{Surface densities}

With the survey selection function from above we were able to compare our number counts of quasars with those of other surveys. We consider two surveys reaching similar or greater depths. The only comparably deep slitless survey is the Palomar Scan Grism Survey (PSGS, Schneider et al. 1999) which yielded 39 quasars on $1.10 \mathrm{deg}^{2}$ at $R \lesssim 22$. Disregarding the known bright quasars at the field centres we detected 102 quasars (known or newly confirmed) and 88 highly promising candidates on $4.39 \mathrm{deg}^{2}$. Assuming that the candidates are quasars, the total surface density for our survey is $\simeq 20 \%$ higher. The wavelength ranges and therefore the redshift ranges are not exactly equal between the two surveys, so that we simply state that the results are in good agreement.

A more quantitative comparison is possible by comparing our survey to the photometric COMBO-17 survey (Wolf et al. 2003). Since COMBO-17 is flux-limited in the $R$ band whereas our survey is best defined in $V$, we assumed a mean quasar colour of $V-R=0.2$ to convert the COMBO-17 surface densities. After correction for the selection function, the cumulative surface number density of quasars found for our survey is $48 \mathrm{deg}^{-2}$ at $V<21$, and $89 \mathrm{deg}^{-2}$ at $V<22$. The corresponding values for COMBO-17 are $51.3 \mathrm{deg}^{-2}$ and $93.5 \mathrm{deg}^{-2}$, respectively. Thus again, the numbers are in excellent agreement, even at magnitudes as faint as $V \sim 22$. Looking closer at the differential number counts we find that they agree very well at $20.5<V<21.5$ (42 \pm 8 in our survey vs. $46 \pm 8$ in COMBO-17), but that our survey drops by a factor of 1.5 in the subsequent magnitude bin at $21.5<V<22.5$ (41 \pm 11 vs. $63 \pm 9$ ). It is not surprising to find that our survey is incomplete at these magnitudes, probably as a combination of incompleteness already in the input catalogues and missing true quasars among the noisy spectra.

As we had included the ECDFS from COMBO-17 in our survey observations, we could also perform an object by object comparison in this well-studied field. The slitless spectra confirmed the general trend of the above survey selection function. We rediscovered 7 out of $10 R<21$ COMBO-17 quasars at $1.3<z<3.6$ located in the common survey area. The three missed quasars are quite faint $(R>20)$ and reside at $z \lesssim 1.9$, where the selection function of our survey drops (Fig. 16). At $z>2.5$ only one out of three $21<R<22$ quasars was rediscovered, indicating photometric incompleteness at the faint end of the DSS catalogue.

\subsection{Comparison to other surveys for quasar groups}

Slitless spectroscopic surveys are successful in finding closely projected quasar groups at high redshift, especially in redshift ranges where colour surveys are less effective. However, previous targeted slitless surveys for quasar groups were few, always pointed at one individual field only (e.g. Jakobsen \& Perryman 1992; Williger et al. 1996), and also generally shallower than our survey by $\sim 1 \mathrm{mag}$. Thus, the resulting surface maps of quasars were sparse, but probed the large-scale distribution of quasars. Our survey significantly increases the number of high-redshift quasar groups with separations $\vartheta \lesssim 15^{\prime}$ in the southern hemisphere. The centroiding of our fields on known bright quasars enables follow-up studies on the three-dimensional distribution of the IGM. Our faint survey limit $\left(V_{\lim }=21-22\right)$ results in a high surface density of high-redshift quasars despite the comparatively small survey area per field.

More recently, Hennawi et al. (2006b) selected faint $i \lesssim 21$ quasar candidates in the vicinity of confirmed SDSS quasars using the SDSS photometry. Their survey was dedicated to find close quasar pairs with separations $\vartheta \lesssim 1^{\prime}$, especially binary quasars at the same redshift. They confirmed 40 new binary quasars and 73 projected quasar pairs. However, 89 of their candidates turned out to be stars, most likely due to broadband colour similarities between stars and quasars in certain redshift ranges. In comparison, our high success rate of almost $100 \%$ shows that slitless spectroscopy is highly efficient in finding high-redshift quasars. A smooth selection function (Fig. 16) is essential to discover high-redshift quasar groups in selected fields. However, given the small surface density of high-redshift quasars, we were unlikely to find quasars at very small separations to the central quasars in our fields. 


\section{Conclusions}

We performed the "Quasars near Quasars" (QNQ) survey, a CCD-based slitless spectroscopic survey for faint $V \lesssim 22$ quasars at $1.7 \lesssim z \lesssim 3.6$ around 18 well-studied bright quasars at $2.76<z<4.69$, covering a total area of $\simeq 4.39 \mathrm{deg}^{2}$. In order to analyse the slitless data we developed a data reduction pipeline that performs an optimal extraction of the spectra and that is able to cope with contaminating spectral orders of slitless grism data. From the 29000 extracted flux-calibrated spectra we selected 169 previously unknown quasar candidates and $>100$ likely low-redshift emission line galaxy candidates on the basis of emission features that fall in the covered wavelength range $4200 \AA \leq \lambda \leq 5800 \AA$. A semi-automatic selection routine limited potential biases of purely visual selection and allowed to quantify selection effects.

Follow-up spectroscopy confirmed 80 out of 81 selected quasar candidates on 16 fields. 64 of these newly established quasars reside at $z>1.7$. The highest redshift quasar is QNQ J22484-6002 at $z=3.586$. The brightest newly discovered high-redshift quasar is QNQ J11197-1340 $(z=2.220$, $B=18.3$ ). Given the high success rate of the follow-up, the vast majority of the remaining 88 candidates will be quasars as well, although most of them likely reside at lower redshifts.

The primary aim of this survey was to provide new groups of quasars for medium-resolution spectroscopy in the vicinity of well-studied known quasars. Originally, we had not expected to reach as faint as $V \sim 22$ in the survey observations. At these faint magnitudes our survey is not well defined because of photometric incompleteness in the source catalogues needed for automatic extraction of the slitless spectra. Together with the fact that not all selected quasar candidates could be included in the spectroscopic follow-up, our survey probably is of limited use for constraining quasar evolution. However, focusing on survey efficiency rather than on completeness at the faint end was justified in order to accomplish the main goal of this survey. In fact, the faintest quasars discovered will for now remain beyond the limits for obtaining high-quality spectra. But at least the $V \lesssim 21$ quasars are well suited for follow-up studies with current (e.g. ESI at Keck) or upcoming (e.g. X-shooter at VLT) high-throughput spectrographs at 8-10 m-class telescopes.

Together with the central quasars in the fields already observed at high resolution, these quasar groups can be used to perform a tomography of the intergalactic medium. Large-scale clustering of the Ly $\alpha$ forest or correlations of metal line systems can be investigated as well (Williger et al. 2000; D'Odorico et al. 2002, 2006). Some of the discovered quasars reside at similar redshifts or approximately at the same redshift of the central quasar in the field, giving potentially insights to quasar clustering along overdense filaments in the plane of the sky. We have identified two quasars coinciding with a damped Ly $\alpha$ absorber on one central line of sight, as well as a large-scale group of quasars at $z=2.70-2.78$. Moreover, our study provides new foreground quasars to investigate the transverse proximity effect of quasars (Papers I and II). In combination with the already available high-resolution spectra of the central quasars, mediumresolution spectra of these quasar groups will offer great opportunities to study the large-scale cosmic web in three dimensions.

Acknowledgements. We thank the staff of the ESO observatories La Silla and Paranal for their professional assistance in obtaining the data presented in this paper. This research has made use of the NASA/IPAC Extragalactic Database (NED) which is operated by the Jet Propulsion Laboratory, California Institute of Technology, under contract with the National Aeronautics and Space Administration. G.W. was partly supported by a HWP grant from the state of
Brandenburg, Germany. G.W. and L.W. acknowledge support by the Deutsche Forschungsgemeinschaft under Wi 1369/21-1.

\section{References}

Adelberger, K. L., Shapley, A. E., Steidel, C. C., et al. 2005, ApJ, 629, 636 Appenzeller, I., Fricke, K., Furtig, W., et al. 1998, The Messenger, 94, 1 Baade, D., Meisenheimer, K., Iwert, O., et al. 1999, The Messenger, 95, 15 Barbieri, C., \& Cristiani, S. 1986, A\&AS, 63, 1

Barger, A. J., Cowie, L. L., Capak, P., et al. 2003, AJ, 126, 632

Becker, G. D., Rauch, M., \& Sargent, W. L. W. 2007, ApJ, 662, 72

Bergeron, J., Petitjean, P., Aracil, B., et al. 2004, The Messenger, 118, 40

Bohuski, T. J., \& Weedman, D. W. 1979, ApJ, 231, 653

Bongiovanni, A., Bruzual, G., Magris, G., et al. 2005, MNRAS, 359, 930

Chand, H., Srianand, R., Petitjean, P., \& Aracil, B. 2004, A\&A, 417, 853

Ciliegi, P., Elvis, M., Gioia, I. M., Maccacaro, T., \& Wolter, A. 1994, AJ, 108, 970

Clowes, R. G., Cooke, J. A., \& Beard, S. M. 1984, MNRAS, 207, 99

Clowes, R. G., Campusano, L. E., \& Graham, M. J. 1999, MNRAS, 309, 48 Cowie, L. L., Barger, A. J., Hu, E. M., Capak, P., \& Songaila, A. 2004, AJ, 127, 3137

Crampton, D., Schade, D., \& Cowley, A. P. 1985, AJ, 90, 987

Crampton, D., Cowley, A. P., \& Hartwick, F. D. A. 1990, AJ, 100, 47

Croom, S. M., Smith, R. J., Boyle, B. J., et al. 2004, MNRAS, 349, 1397

Crotts, A. P. S., \& Fang, Y. 1998, ApJ, 502, 16

Dinshaw, N., \& Impey, C. D. 1996, ApJ, 458, 73

D'Odorico, V., Petitjean, P., Cristiani, S., \& D'Odorico, S. 2002, A\&A, 390, 13

D'Odorico, V., Viel, M., Saitta, F., et al. 2006, MNRAS, 372, 1333

Francis, P. J., Palunas, P., Teplitz, H. I., Williger, G. M., \& Woodgate, B. E. 2004, ApJ, 614, 75

Gaskell, C. M. 1982, ApJ, 263, 79

Gratton, R. G., \& Osmer, P. S. 1987, PASP, 99, 899

Hawkins, M. R. S. 2000, A\&AS, 143, 465

Hazard, C., \& McMahon, R. 1985, Nature, 314, 238

Hazard, C., Morton, D. C., McMahon, R. G., Sargent, W. L. W., \& Terlevich, R. 1986, MNRAS, 223, 87

Hazard, C., McMahon, R. G., \& Morton, D. C. 1987, MNRAS, 229, 371

Heap, S. R., Williger, G. M., Smette, A., et al. 2000, ApJ, 534, 69

Hennawi, J. F., \& Prochaska, J. X. 2007, ApJ, 655, 735

Hennawi, J. F., Prochaska, J. X., Burles, S., et al. 2006a, ApJ, 651, 61

Hennawi, J. F., Strauss, M. A., Oguri, M., et al. 2006b, AJ, 131, 1

Hewett, P. C., Irwin, M. J., Bunclark, P., et al. 1985, MNRAS, 213, 971

Hewett, P. C., Foltz, C. B., \& Chaffee, F. H. 1995, AJ, 109, 1498

Hewitt, A., \& Burbidge, G. 1993, ApJS, 87, 451

Hook, I. M., Shaver, P. A., Jackson, C. A., Wall, J. V., \& Kellermann, K. I. 2003, A\&A, 399, 469

Horne, K. 1986, PASP, 98, 609

Jakobsen, P., \& Perryman, M. A. C. 1992, ApJ, 392, 432

Jakobsen, P., Perryman, M. A. C., Ulrich, M. H., Macchetto, F., \& Di Serego Alighieri, S. 1986, ApJ, 303, L27

Jakobsen, P., Boksenberg, A., Deharveng, J. M., et al. 1994, Nature, 370, 35 Jakobsen, P., Jansen, R. A., Wagner, S., \& Reimers, D. 2003, A\&A, 397, 891 Jauncey, D. L., Wright, A. E., Peterson, B. A., \& Condon, J. J. 1978, ApJ, 219, L1

Jiang, L., Fan, X., Annis, J., et al. 2008, AJ, 135, 1057

Kim, T.-S., Bolton, J. S., Viel, M., Haehnelt, M. G., \& Carswell, R. F. 2007, MNRAS, 382, 1657

Krumpe, M., Lamer, G., Schwope, A. D., et al. 2007, A\&A, 466, 41

La Franca, F., Lissandrini, C., Cristiani, S., et al. 1999, A\&AS, 140, 351

Landolt, A. U. 1992, AJ, 104, 340

Liske, J., Webb, J. K., Williger, G. M., Fernández-Soto, A., \& Carswell, R. F. 2000, MNRAS, 311, 657

Lopez, S., Reimers, D., D’Odorico, S., \& Prochaska, J. X. 2002, A\&A, 385, 778

Maza, J., Ruiz, M. T., González, L. E., Wischnjewsky, M., \& Antezana, R. 1993, RMxAA, 25, 51

Maza, J., Wischnjewsky, M., Antezana, R., \& González, L. E. 1995, RMxAA, 31,119

McIntosh, D. H., Rix, H.-W., Rieke, M. J., \& Foltz, C. B. 1999, ApJ, 517, L73

McMahon, R. G., Omont, A., Bergeron, J., Kreysa, E., \& Haslam, C. G. T. 1994, MNRAS, 267, L9

Morris, S. L., Weymann, R. J., Anderson, S. F., et al. 1991, AJ, 102, 1627

Murphy, M. T., Webb, J. K., \& Flambaum, V. V. 2003, MNRAS, 345, 609

Osmer, P. S. 1980, ApJS, 42, 523

Osmer, P. S., \& Smith, M. G. 1976, ApJ, 210, 267

Osmer, P. S., \& Smith, M. G. 1980, ApJS, 42, 333

Pascual, S., Gallego, J., Aragón-Salamanca, A., \& Zamorano, J. 2001, A\&A, 379,798 
Pichon, C., Vergely, J. L., Rollinde, E., Colombi, S., \& Petitjean, P. 2001, MNRAS, 326, 597

Prescott, M. K. M., Impey, C. D., Cool, R. J., \& Scoville, N. Z. 2006, ApJ, 644, 100

Reimers, D., Rodriguez-Pascual, P., Hagen, H.-J., \& Wisotzki, L. 1995, A\&A, 293, L21

Reimers, D., Köhler, S., Wisotzki, L., et al. 1997, A\&A, 327, 890

Richards, G. T., Fan, X., Newberg, H. J., et al. 2002a, AJ, 123, 2945

Richards, G. T., Vanden Berk, D. E., Reichard, T. A., et al. 2002b, AJ, 124, 1

Salzer, J. J., Gronwall, C., Sarajedini, V. L., et al. 2002, AJ, 123, 1292

Schmidt, M., Schneider, D. P., \& Gunn, J. E. 1986, ApJ, 306, 411

Schmidt, M., Schneider, D. P., \& Gunn, J. E. 1995, AJ, 110, 68

Schneider, D. P., Schmidt, M., \& Gunn, J. E. 1994, AJ, 107, 1245

Schneider, D. P., Schmidt, M., \& Gunn, J. E. 1999, AJ, 117, 40

Schneider, D. P., Hall, P. B., Richards, G. T., et al. 2007, AJ, 134, 102

Sharp, R. G., Sabbey, C. N., Vivas, A. K., et al. 2002, MNRAS, 337, 1153

Shaver, P. 1987, Nature, 330, 426

Smette, A., Heap, S. R., Williger, G. M., et al. 2002, ApJ, 564, 542

Sramek, R. A., \& Weedman, D. W. 1978, ApJ, 221, 468

Steidel, C. C., Adelberger, K. L., Shapley, A. E., et al. 2003, ApJ, 592, 728

Storrie-Lombardi, L. J., McMahon, R. G., Irwin, M. J., \& Hazard, C. 1996, ApJ, 468, 121
Szokoly, G. P., Bergeron, J., Hasinger, G., et al. 2004, ApJS, 155, 271

Trump, J. R., Impey, C. D., McCarthy, P. J., et al. 2007, ApJS, 172, 383

Tytler, D., \& Fan, X. 1992, ApJS, 79, 1

Vanden Berk, D. E., Richards, G. T., Bauer, A., et al. 2001, AJ, 122, 549

Véron, P., \& Hawkins, M. R. S. 1995, A\&A, 296, 665

Warren, S. J., Hewett, P. C., Irwin, M. J., \& Osmer, P. S. 1991, ApJS, 76, 1

Williger, G. M., Hazard, C., Baldwin, J. A., \& McMahon, R. G. 1996, ApJS, 104,145

Williger, G. M., Smette, A., Hazard, C., Baldwin, J. A., \& McMahon, R. G. 2000, ApJ, 532, 77

Wisotzki, L., Christlieb, N., Bade, N., et al. 2000, A\&A, 358, 77

Wisotzki, L., Selman, F., \& Gilliotte, A. 2001, The Messenger, 104, 8

Wolf, C., Wisotzki, L., Borch, A., et al. 2003, A\&A, 408, 499

Wolf, C., Meisenheimer, K., Kleinheinrich, M., et al. 2004, A\&A, 421, 913

Worseck, G., \& Wisotzki, L. 2006, A\&A, 450, 495

Worseck, G., Fechner, C., Wisotzki, L., \& Dall' Aglio, A. 2007, A\&A, 473, 805

Wright, A. E., Jauncey, D. L., Peterson, B. A., \& Condon, J. J. 1977, ApJ, 211, L115

Zheng, W., Kriss, G. A., Deharveng, J.-M., et al. 2004, ApJ, 605, 631

Zitelli, V., Mignoli, M., Zamorani, G., Marano, B., \& Boyle, B. J. 1992, MNRAS, 256, 349 
G. Worseck et al.: A slitless spectroscopic survey for quasars near quasars, Online Material $p 1$

Appendix A: Follow-up FORS2 spectra
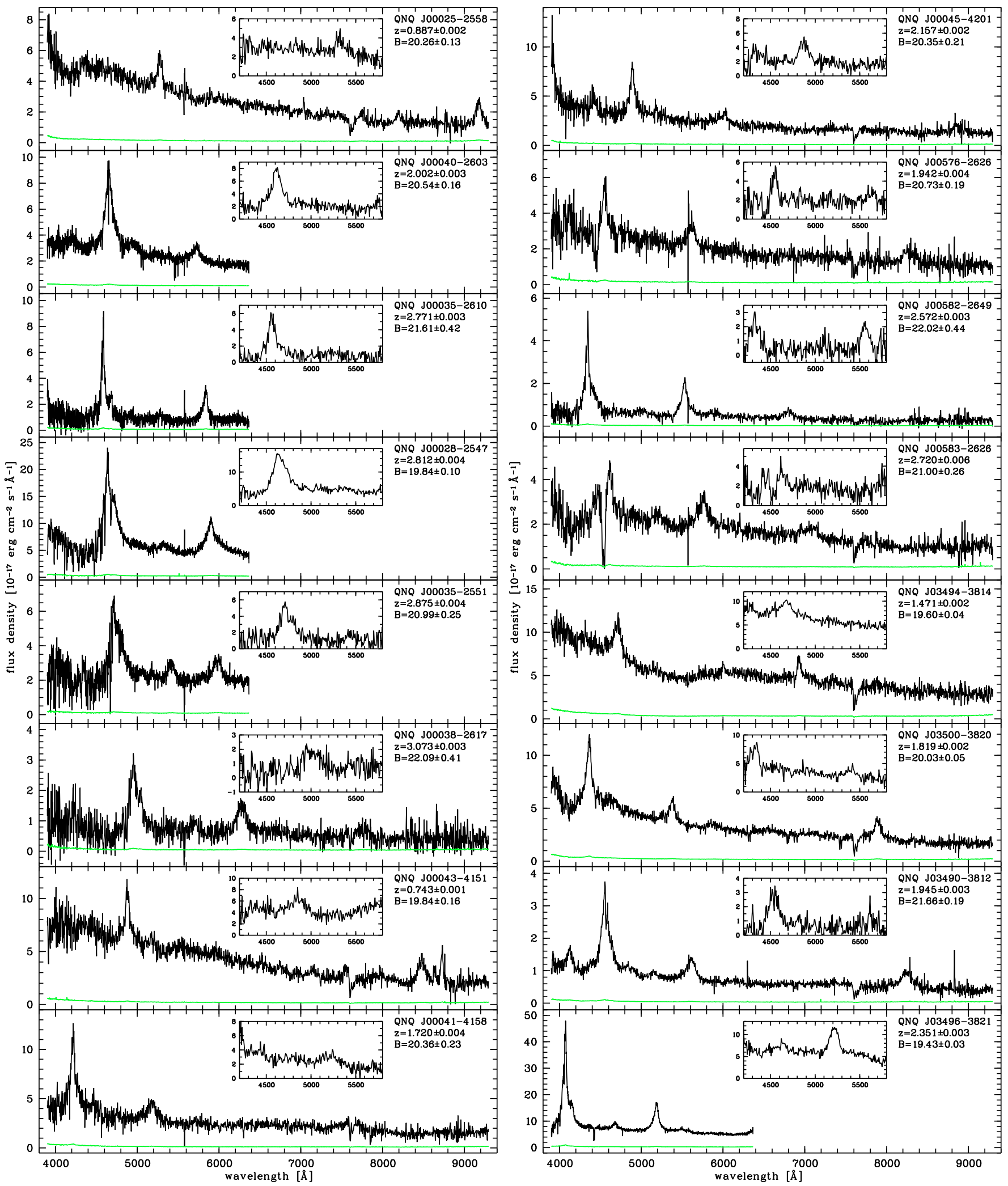

Fig. A. 1. VLT/FORS2 spectra (black) and $1 \sigma$ noise arrays (green) of 57 quasars discovered in the QNQ survey. Spectra presented in Worseck \& Wisotzki (2006), Worseck et al. (2007) and in Fig. 12 of this paper are not shown. The spectra have been scaled to yield the measured photometric $B$ magnitudes. The small inserts show the corresponding slitless WFI spectra in the same units. The spectra are ordered columnwise by right ascension of the field and redshift of the quasar. 
G. Worseck et al.: A slitless spectroscopic survey for quasars near quasars, Online Material p 2
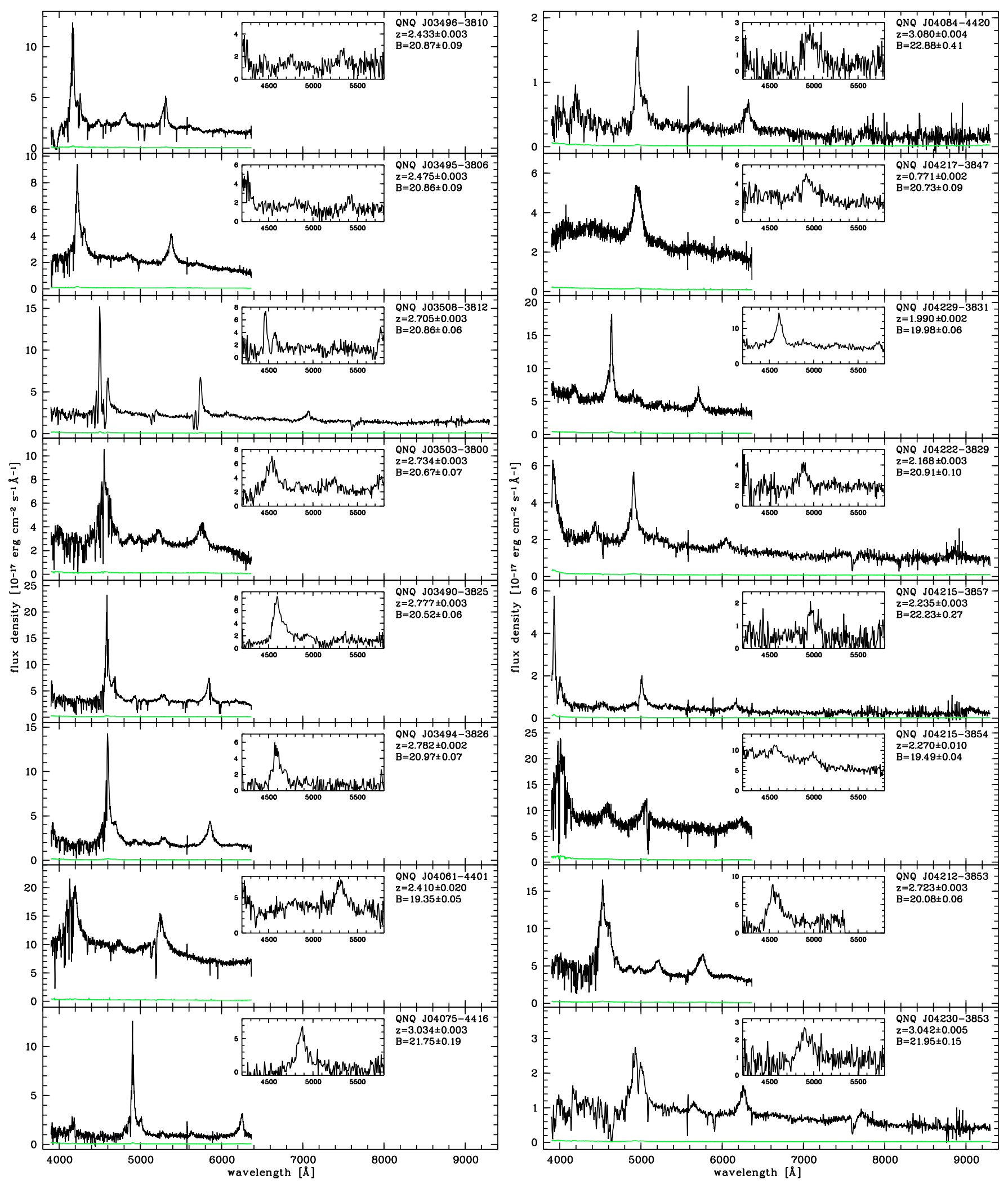

Fig. A. 1. continued. 
G. Worseck et al.: A slitless spectroscopic survey for quasars near quasars, Online Material p 3
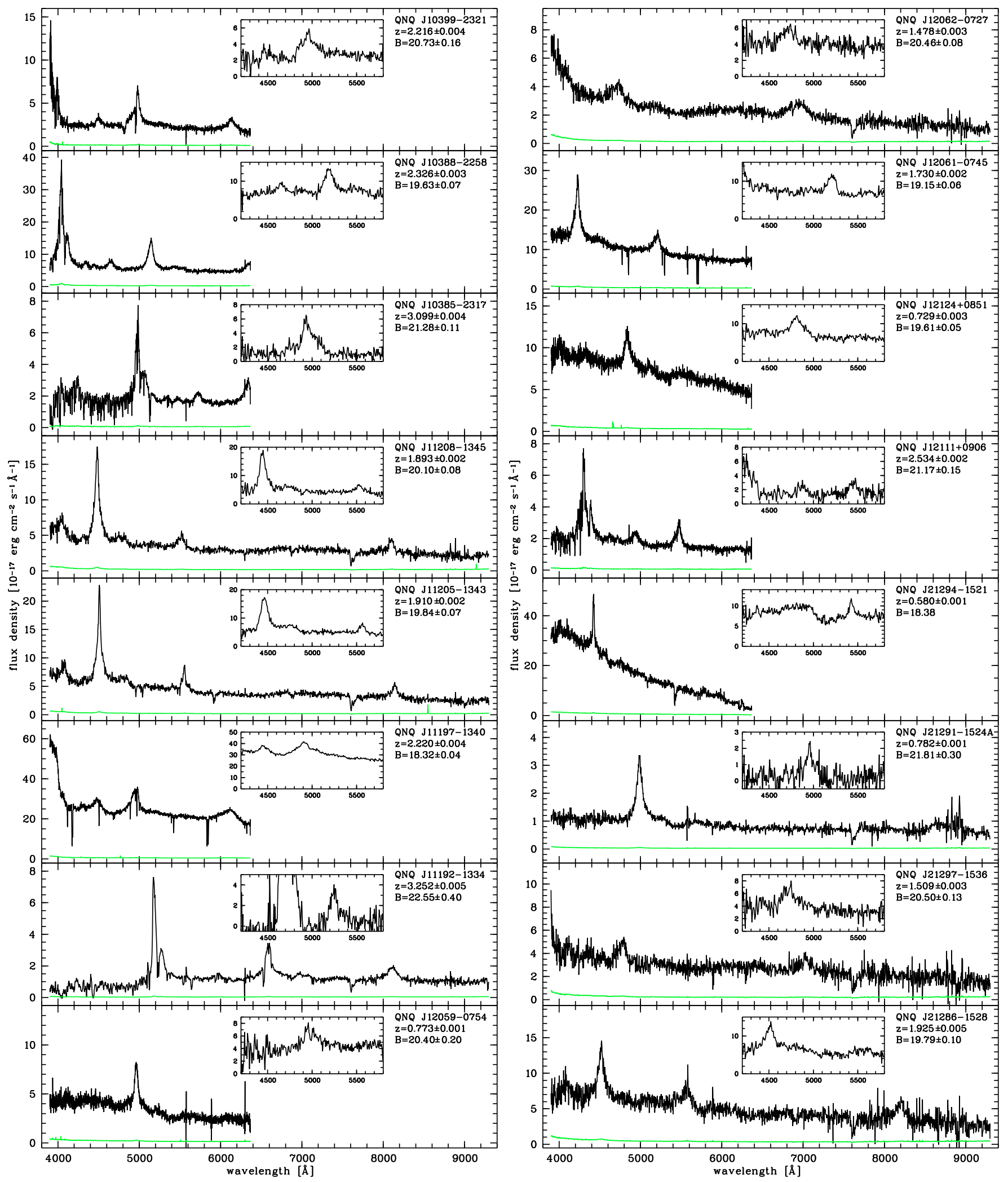

Fig. A. 1. continued. 
G. Worseck et al.: A slitless spectroscopic survey for quasars near quasars, Online Material p 4
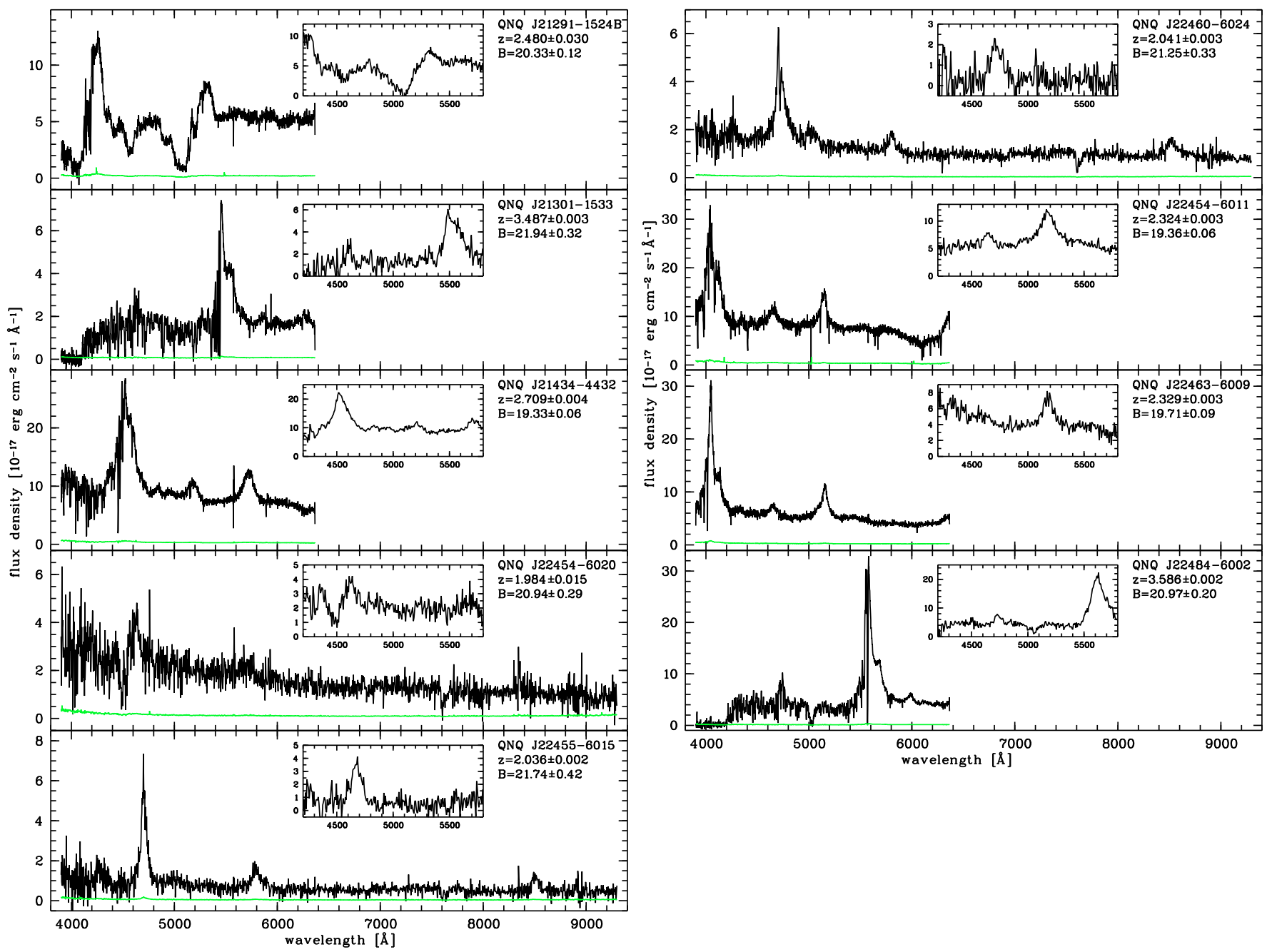

Fig. A. 1. continued. 
G. Worseck et al.: A slitless spectroscopic survey for quasars near quasars, Online Material p 5

\section{Appendix B: Quasar candidates not included in the spectroscopic follow-up}

Table B. 1. Remaining quasar candidates not included in the spectroscopic follow-up. The columns list the central quasar in the field, the object (format: HHMMSS. S \pm DDMMSS, J2000), the approximate $V$ magnitude and the redshift with redshift confidence.

\begin{tabular}{|c|c|c|c|c|c|c|c|c|c|}
\hline Field & Object & $V$ & $z$ & $z$ confidence & Field & Object & $V$ & $z$ & $z$ confidence \\
\hline Q 0000-263 & J000239.4-261139 & 22.0 & 0.68 & uncertain & HE $0940-1050$ & J094223.0-110952 & 19.1 & 0.67 & plausible \\
\hline Q 0000-263 & J000256.4-260956 & 21.2 & 1.77 & plausible & HE $0940-1050$ & J094228.1-111403 & 20.5 & 1.38 & plausible \\
\hline Q $0000-263$ & J000256.8-254703 & 21.4 & 0.91 & plausible & HE $0940-1050$ & J094228.1-104719 & 21.5 & 2.70 & uncertain \\
\hline Q $0000-263$ & J000257.9-255750 & 21.1 & 1.79 & secure & HE $0940-1050$ & J094233.4-112119 & 20.5 & 1.24 & uncertain \\
\hline Q 0000-263 & J000404.9-254841 & 22.5 & 0.68 & uncertain & HE $0940-1050$ & J094324.7-111526 & 17.6 & 0.99 & secure \\
\hline Q $0000-263$ & J000408.5-255024 & 21.5 & 0.71 & uncertain & HE $0940-1050$ & J094336.0-110129 & 19.5 & 1.70 & plausible \\
\hline Q 0000-263 & J000422.1-255830 & 20.6 & 0.91 & plausible & HE $0940-1050$ & J094342.5-111730 & 19.0 & 0.90 & secure \\
\hline Q 0002-422 & J000531.8-415410 & 20.6 & 1.07 & secure & CTQ 0460 & $\mathrm{~J} 103833.6-232642$ & 19.9 & 1.93 & uncertain \\
\hline Q $0055-269$ & J005709.5-265900 & 20.8 & 0.70 & uncertain & CTQ 0460 & J103836.2-231020 & 21.9 & 2.36 & plausible \\
\hline Q 0055-269 & J005750.3-263910 & 20.6 & 1.41 & secure & CTQ 0460 & J103903.6-225625 & 19.9 & 1.92 & plausible \\
\hline Q $0055-269$ & J005805.6-265755 & 23.0 & 2.88 & uncertain & BR $1117-1329$ & J111910.3-133139 & 21.4 & 1.55 & uncertain \\
\hline Q 0055-269 & J005840.0-263314 & 19.5 & 2.55 & plausible & BR 1117-1329 & J111924.7-135152 & 20.8 & 1.29 & uncertain \\
\hline Q 0055-269 & J005847.4-264308 & 21.0 & 0.75 & uncertain & BR 1117-1329 & J111946.1-134157 & 20.0 & 0.84 & plausible \\
\hline Q $0347-383$ & J034856.4-380847 & 21.2 & 1.83 & uncertain & BR 1117-1329 & J112028.1-133612 & 20.5 & 1.30 & uncertain \\
\hline Q $0347-383$ & J034905.8-380146 & 21.9 & 1.84 & secure & BR 1117-1329 & J112037.2-133603 & 18.6 & 0.15 & secure \\
\hline Q 0347-383 & J034933.3-381618 & 19.5 & 0.94 & secure & BR 1117-1329 & J112049.4-133422 & 21.2 & 2.84 & plausible \\
\hline Q $0347-383$ & J034945.9-380150 & 20.9 & 1.85 & secure & BR 1202-0725 & J120506.0-075537 & 18.6 & 1.58 & secure \\
\hline Q $0347-383$ & J035003.7-375855 & 22.0 & 2.08 & uncertain & BR $1202-0725$ & J120512.7-075926 & 20.5 & 3.32 & uncertain \\
\hline Q 0347-383 & J035008.5-375359 & 20.8 & 1.84 & secure & BR 1202-0725 & J120601.9-073433 & 21.6 & 2.11 & uncertain \\
\hline Q 0347-383 & J035039.4-382425 & 19.4 & 1.38 & plausible & BR 1202-0725 & $\mathrm{J} 120602.2-074358$ & 20.0 & 0.84 & plausible \\
\hline CTQ 0247 & J040634.9-440757 & 19.6 & 1.37 & plausible & BR $1202-0725$ & J120612.2-072814 & 20.4 & 0.71 & uncertain \\
\hline CTQ 0247 & J040637.7-442152 & 21.1 & 1.68 & uncertain & Q 1209+093 & $\mathrm{J} 121108.4+085707$ & 20.6 & 0.86 & uncertain \\
\hline CTQ 0247 & J040706.6-442223 & 20.5 & 1.68 & uncertain & Q 1209+093 & $\mathrm{J} 121234.8+085701$ & 20.5 & 2.17 & uncertain \\
\hline CTQ 0247 & J040735.1-440020 & 18.8 & 1.47 & plausible & Q $1451+123$ & $\mathrm{~J} 145508.6+121245$ & 20.5 & 1.68 & uncertain \\
\hline CTQ 0247 & J040744.2-440050 & 19.8 & 0.59 & plausible & Q $1451+123$ & $\mathrm{~J} 145514.6+115655$ & 21.5 & 2.04 & uncertain \\
\hline CTQ 0247 & J040816.1-442158 & 20.5 & 0.91 & uncertain & PKS 2126-158 & J212924.5-154820 & 18.5 & 1.57 & secure \\
\hline CTQ 0247 & J040822.8-441814 & 21.5 & 1.76 & plausible & PKS 2126-158 & J213009.8-153633 & 22.0 & 2.41 & plausible \\
\hline Q 0420-388 & J042116.9-384010 & 22.0 & 2.41 & uncertain & PKS 2126-158 & J213016.1-154153 & 20.2 & 0.77 & uncertain \\
\hline Q 0420-388 & J042145.7-385720 & 21.6 & 1.79 & secure & PKS 2126-158 & $\mathrm{J} 213017.3-154324$ & 18.5 & 1.41 & secure \\
\hline Q 0420-388 & J042153.2-383256 & 20.5 & 0.86 & plausible & Q 2139-4434 & J214119.4-441256 & 21.6 & 1.57 & uncertain \\
\hline Q 0420-388 & J042222.0-385755 & 21.5 & 2.92 & plausible & Q 2139-4434 & $\mathrm{J} 214124.0-443301$ & 21.0 & 1.96 & uncertain \\
\hline Q 0420-388 & J042258.9-384749 & 20.8 & 0.89 & plausible & Q 2139-4434 & $\mathrm{J} 214326.2-442036$ & 18.2 & 0.60 & uncertain \\
\hline PKS 0528-250 & J052916.6-245655 & 21.1 & 2.34 & plausible & HE 2243-6031 & $\mathrm{J} 224542.2-600645$ & 20.0 & 1.53 & secure \\
\hline PKS 0528-250 & J052920.1-251337 & 20.5 & 2.20 & uncertain & HE 2243-6031 & J224543.0-603049 & 19.4 & 1.56 & secure \\
\hline PKS 0528-250 & J052930.5-245305 & 18.5 & 0.86 & secure & HE 2243-6031 & J224553.3-600659 & 20.2 & 1.36 & secure \\
\hline PKS 0528-250 & J052939.1-250148 & 20.2 & 2.85 & secure & HE 2243-6031 & $\mathrm{J} 224602.9-603115$ & 21.0 & 0.66 & uncertain \\
\hline PKS 0528-250 & J052940.7-245927 & 21.5 & 1.72 & plausible & HE 2243-6031 & J224612.7-600355 & 21.9 & 2.91 & uncertain \\
\hline PKS 0528-250 & J052947.4-250426 & 20.0 & 2.88 & secure & HE 2243-6031 & $\mathrm{J} 224653.0-603046$ & 20.5 & 0.62 & uncertain \\
\hline PKS 0528-250 & J053015.5-250307 & 21.1 & 2.52 & secure & HE 2243-6031 & J224729.0-601443 & 20.8 & 0.93 & secure \\
\hline PKS 0528-250 & J053039.7-245306 & 21.9 & 1.53 & uncertain & HE 2243-6031 & $\mathrm{J} 224750.4-602117$ & 19.5 & 1.02 & plausible \\
\hline PKS 0528-250 & J053043.2-250840 & 21.4 & 1.84 & plausible & HE 2243-6031 & J224837.7-601431 & 21.4 & 3.08 & uncertain \\
\hline PKS 0528-250 & J053048.2-250435 & 20.6 & 2.95 & plausible & HE 2347-4342 & J235001.9-431229 & 18.6 & 0.92 & secure \\
\hline PKS 0528-250 & J053115.3-251311 & 21.0 & 1.41 & uncertain & HE $2347-4342$ & J235107.0-432246 & 19.0 & 1.48 & plausible \\
\hline HE $0940-1050$ & J094219.2-105019 & 20.9 & 0.94 & plausible & HE 2347-4342 & $\mathrm{J} 235140.8-431615$ & 20.9 & 1.26 & uncertain \\
\hline
\end{tabular}


G. Worseck et al.: A slitless spectroscopic survey for quasars near quasars, Online Material p 6
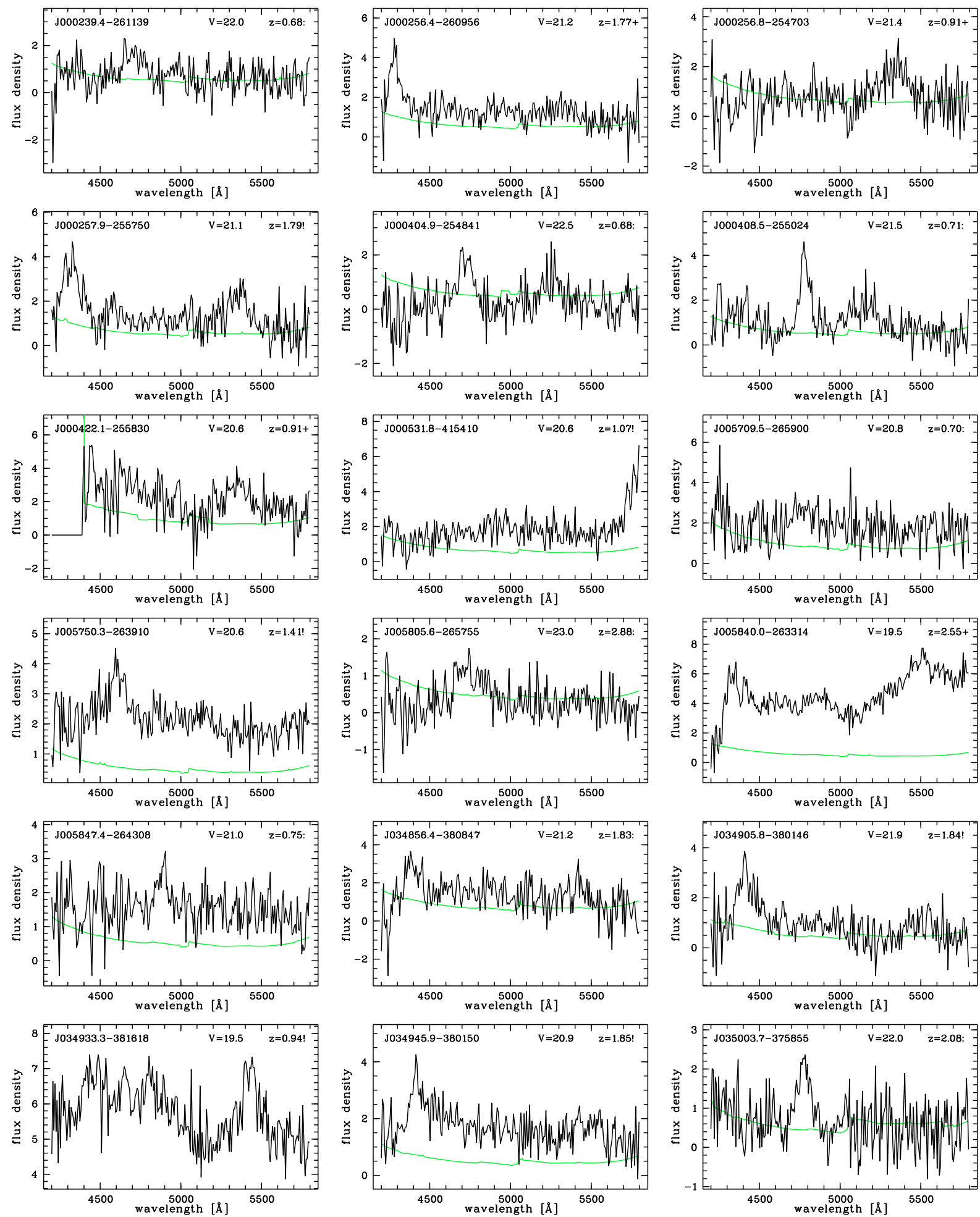

Fig. B. 1. RA-ordered list of slitless WFI spectra of 88 unknown quasar candidates without follow-up spectra. The spectra are shown in black together with their $1 \sigma$ noise arrays (green/grey) if the $S / N$ is low. The (approximate) flux densities are given in $10^{-17} \mathrm{erg} \mathrm{cm}^{-2} \mathrm{~s}^{-1} \AA^{-1}$. Labelled are the celestial coordinates (format: HHMMSS. S \pm DDMMSS, J2000), the integrated $V$ magnitude and the estimated redshift. Ambiguous redshifts guessed from a single emission line are marked by a colon. Plus signs mark plausible redshift estimates. Exclamation marks stand for almost secure redshifts based on the shape of a single line or the detection of two lines either in the principal spectral range $4200 \AA \leq \lambda \leq 5800 \AA$ or the $R$ band spectra if available. J005840.0-263314 is a prominent BAL QSO. 
G. Worseck et al.: A slitless spectroscopic survey for quasars near quasars, Online Material $p 7$
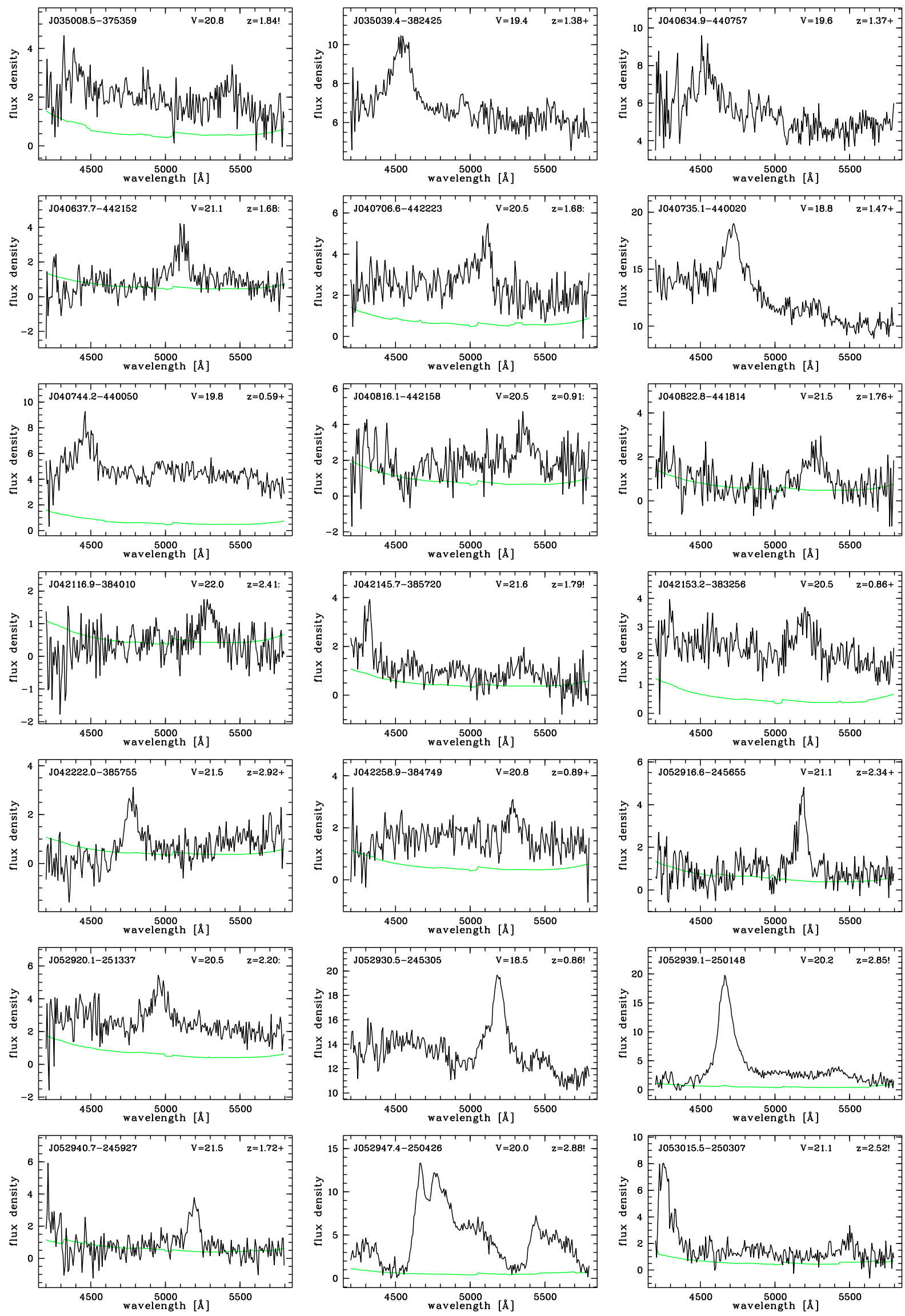

Fig. B. 1. continued. Note that the field of PKS 0528-250 with 7 bona fide quasar candidates was not included in the follow-up campaign with FORS2. 
G. Worseck et al.: A slitless spectroscopic survey for quasars near quasars, Online Material $p 8$
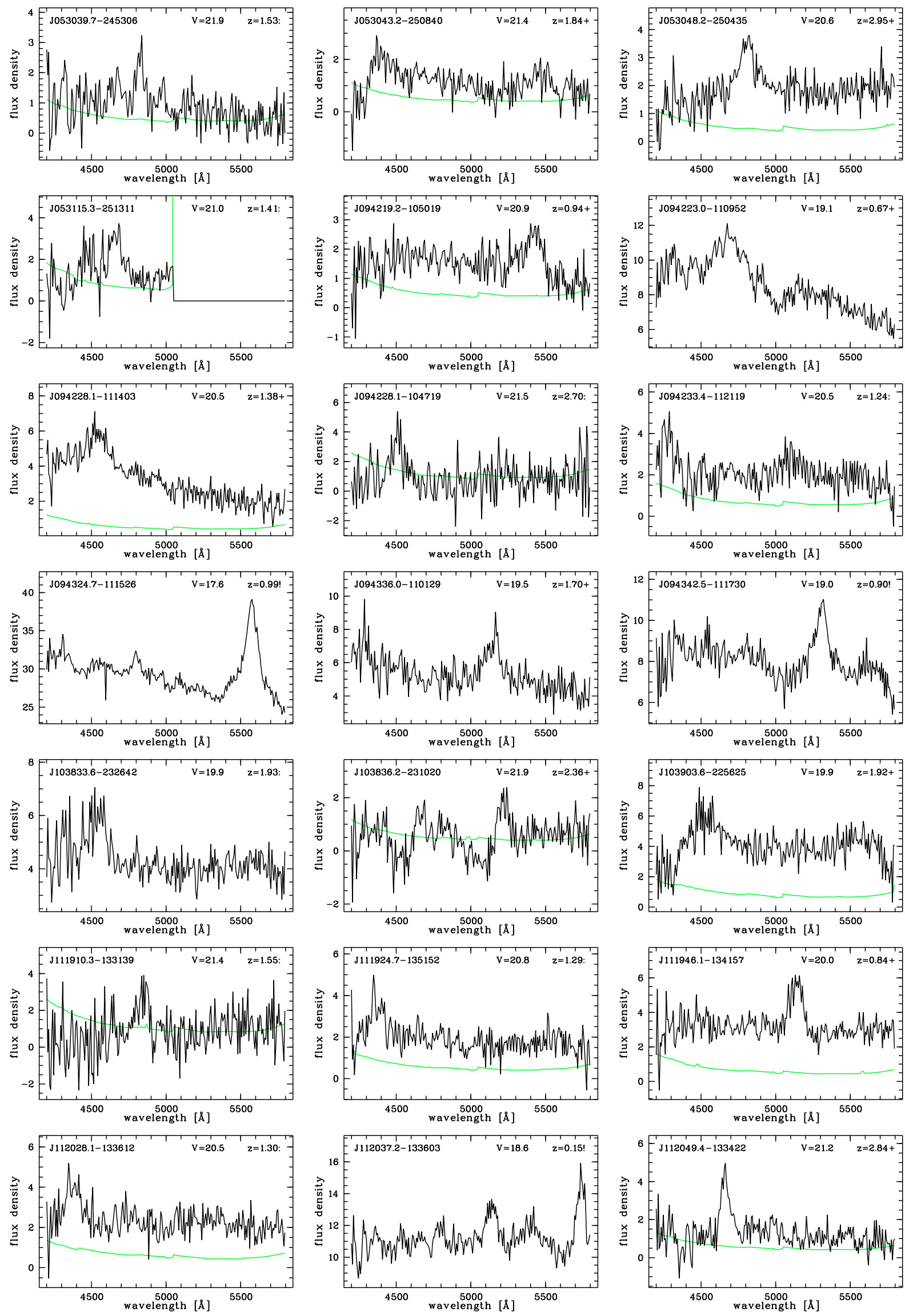

Fig. B. 1. continued. 
G. Worseck et al.: A slitless spectroscopic survey for quasars near quasars, Online Material $p 9$
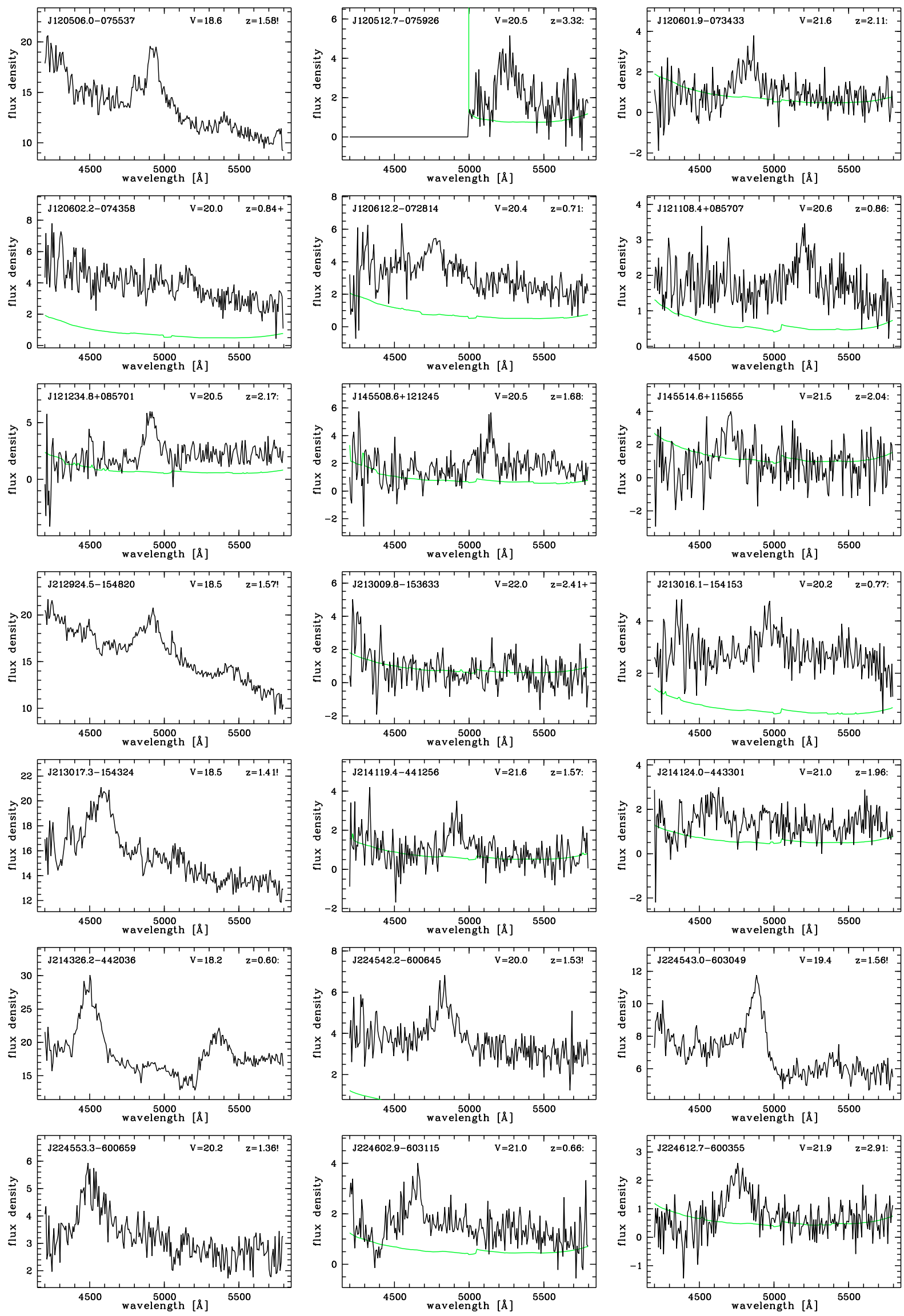

Fig. B. 1. continued. The spectrum of J214326.2-442036 might be contaminated by a nearby galaxy. Francis et al. (2004) list J214124.0-443301 as a quasar candidate with uncertain redshift, whereas they identified J214119.4-441256 as a galaxy at $z=0.062$ which is not supported by the slitless WFI spectrum. 
G. Worseck et al.: A slitless spectroscopic survey for quasars near quasars, Online Material p 10
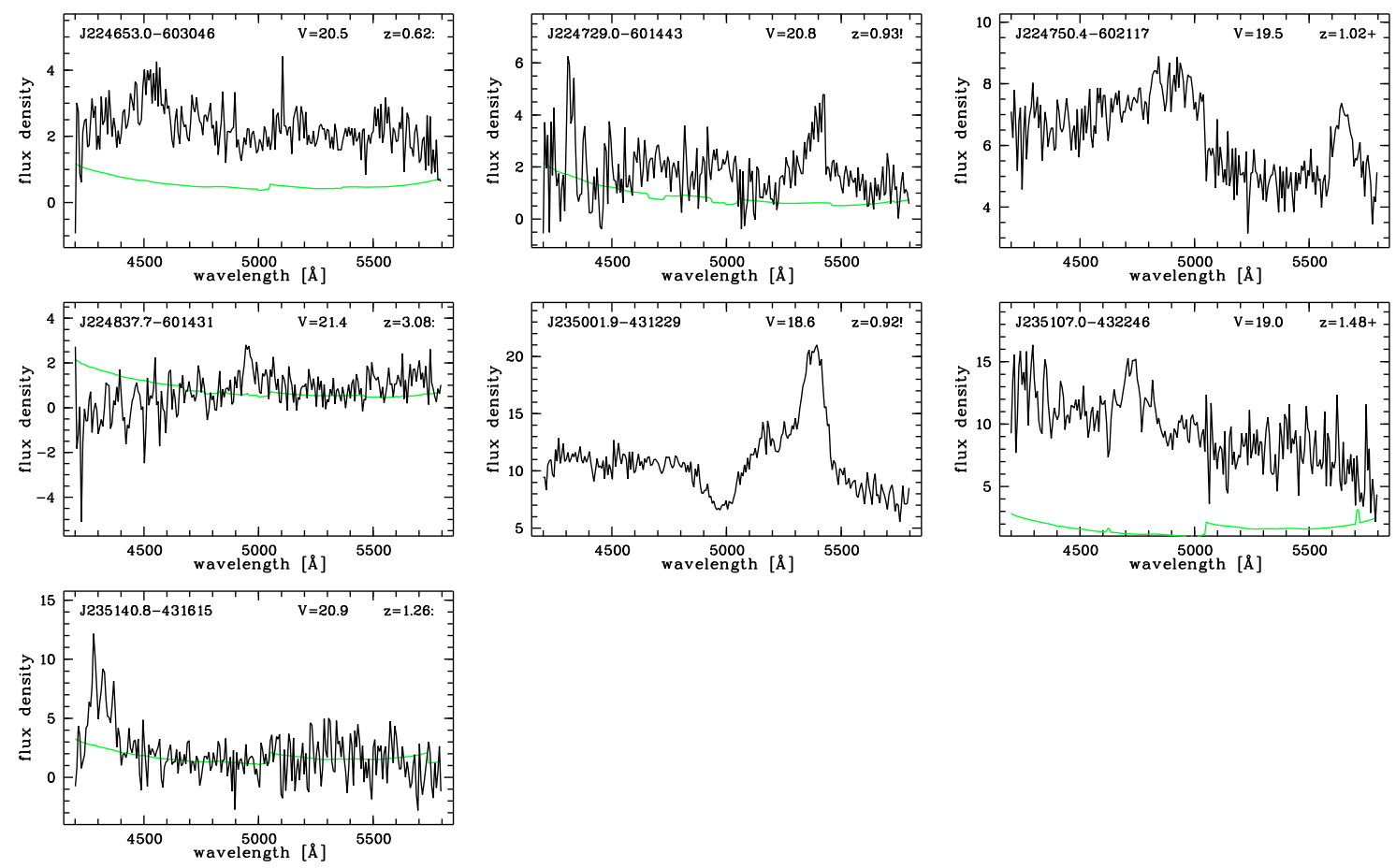

Fig. B. 1. continued. 
G. Worseck et al.: A slitless spectroscopic survey for quasars near quasars, Online Material p 11

\section{Appendix C: Quasar fields}
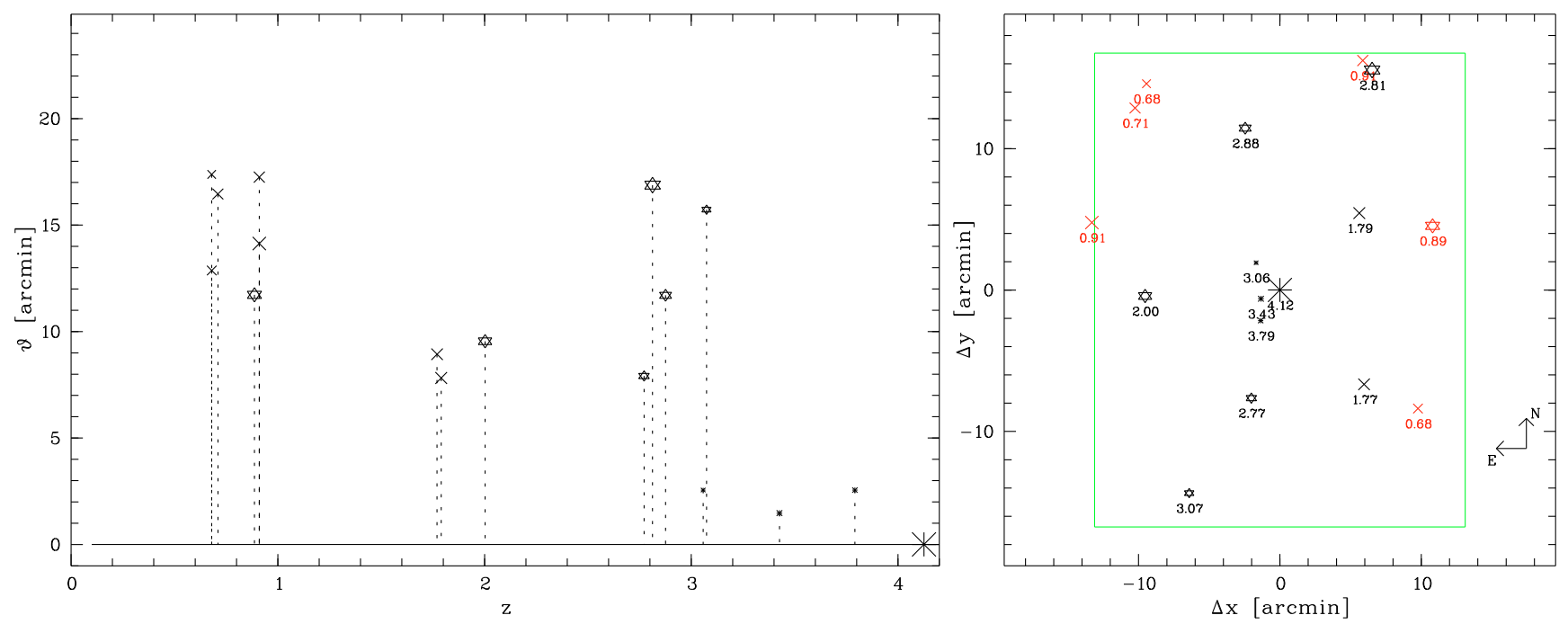

Fig. C. 1. As Fig. 15 for the field centred near Q 0000-263.
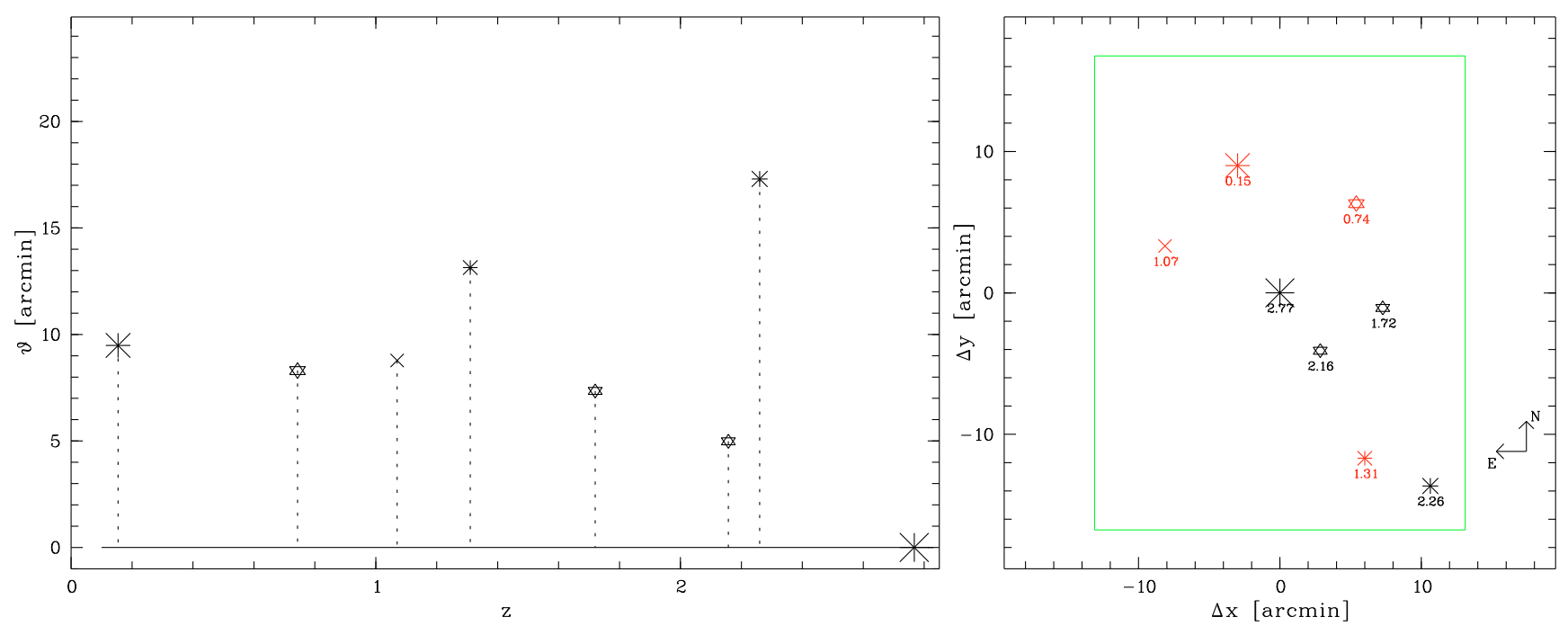

Fig. C. 2. As Fig. 15 for the field centred near Q 0002-422.
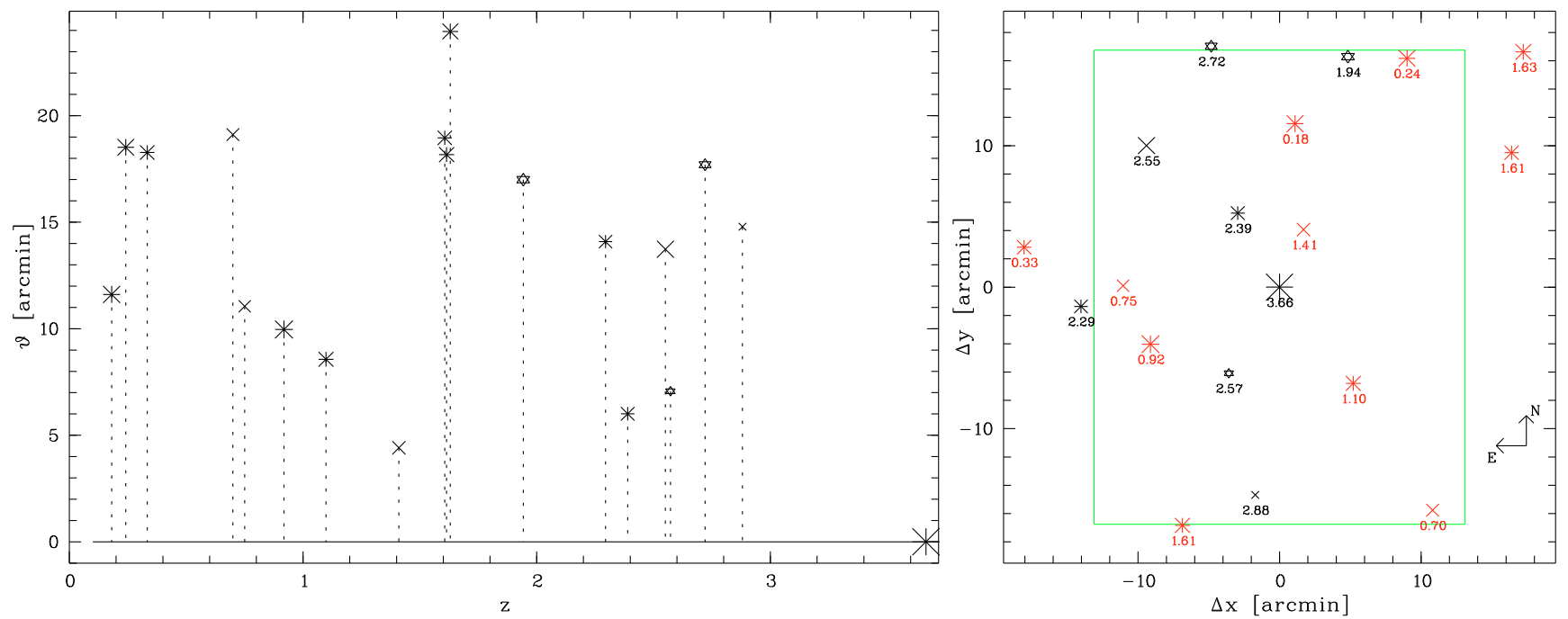

Fig. C. 3. As Fig. 15 for the field centred near Q 0055-269. 
G. Worseck et al.: A slitless spectroscopic survey for quasars near quasars, Online Material p 12
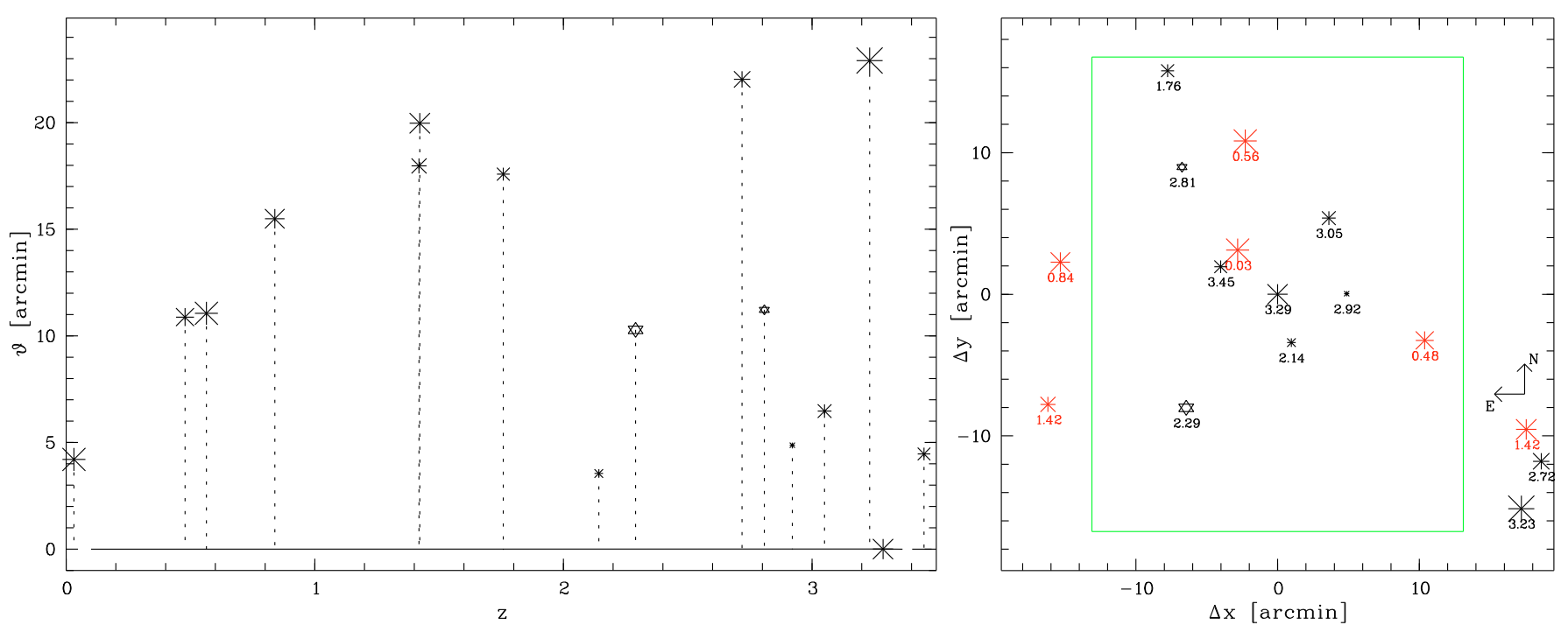

Fig. C. 4. As Fig. 15 for the field centred near Q 0302-003.
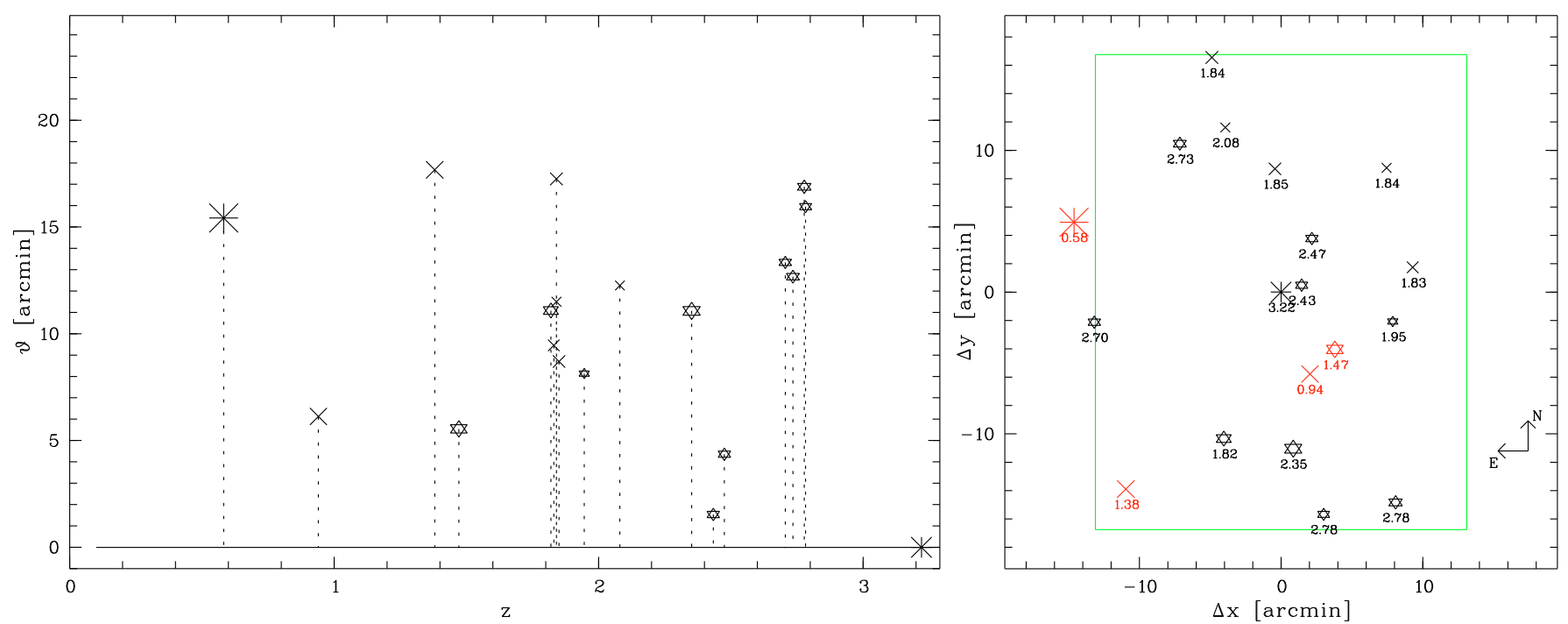

Fig. C. 5. As Fig. 15 for the field centred near Q 0347-383.
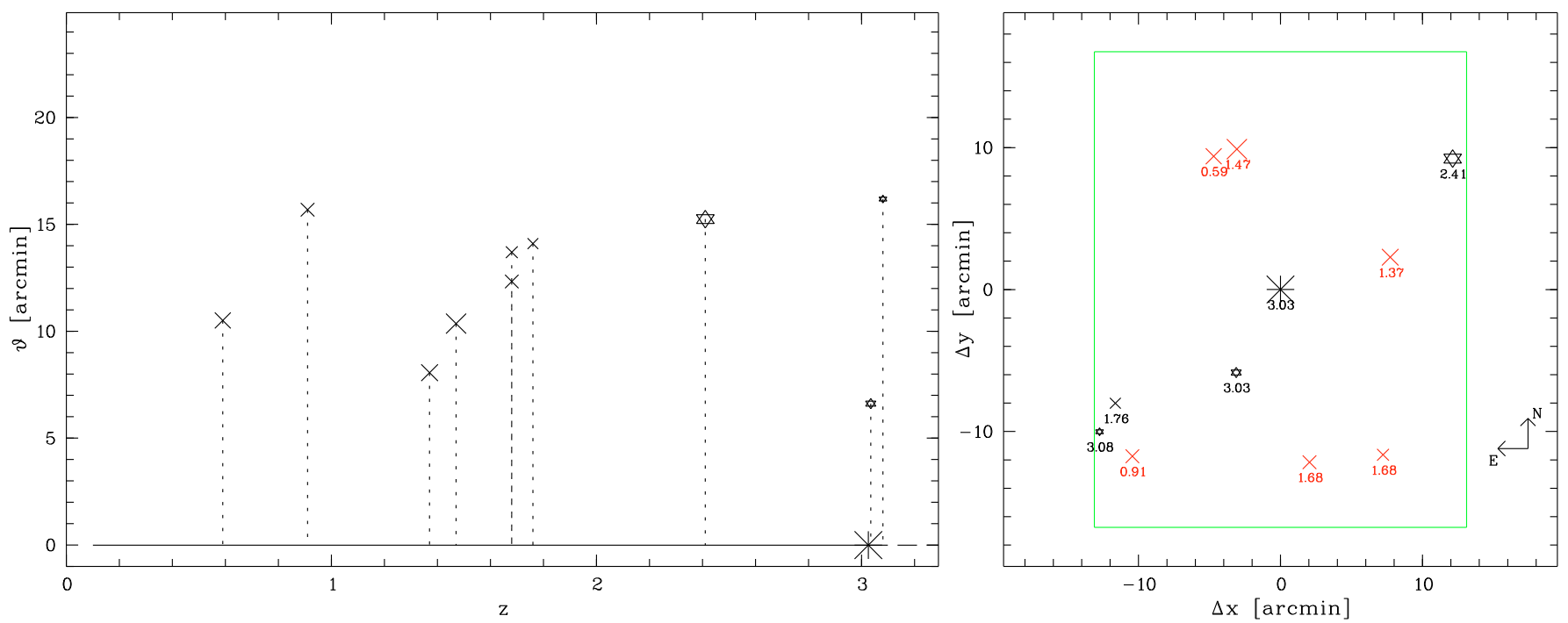

Fig. C. 6. As Fig. 15 for the field centred near CTQ 0247. 
G. Worseck et al.: A slitless spectroscopic survey for quasars near quasars, Online Material p 13
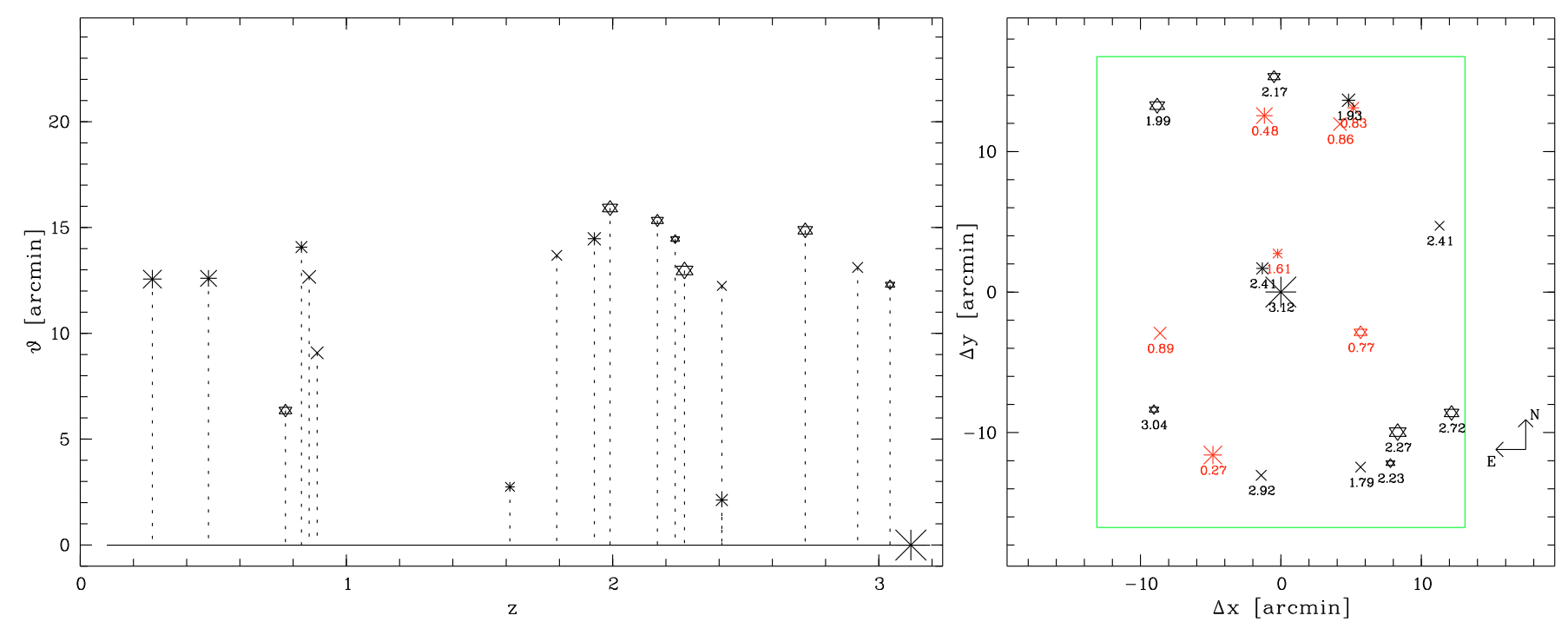

Fig. C. 7. As Fig. 15 for the field centred near Q 0420-388.
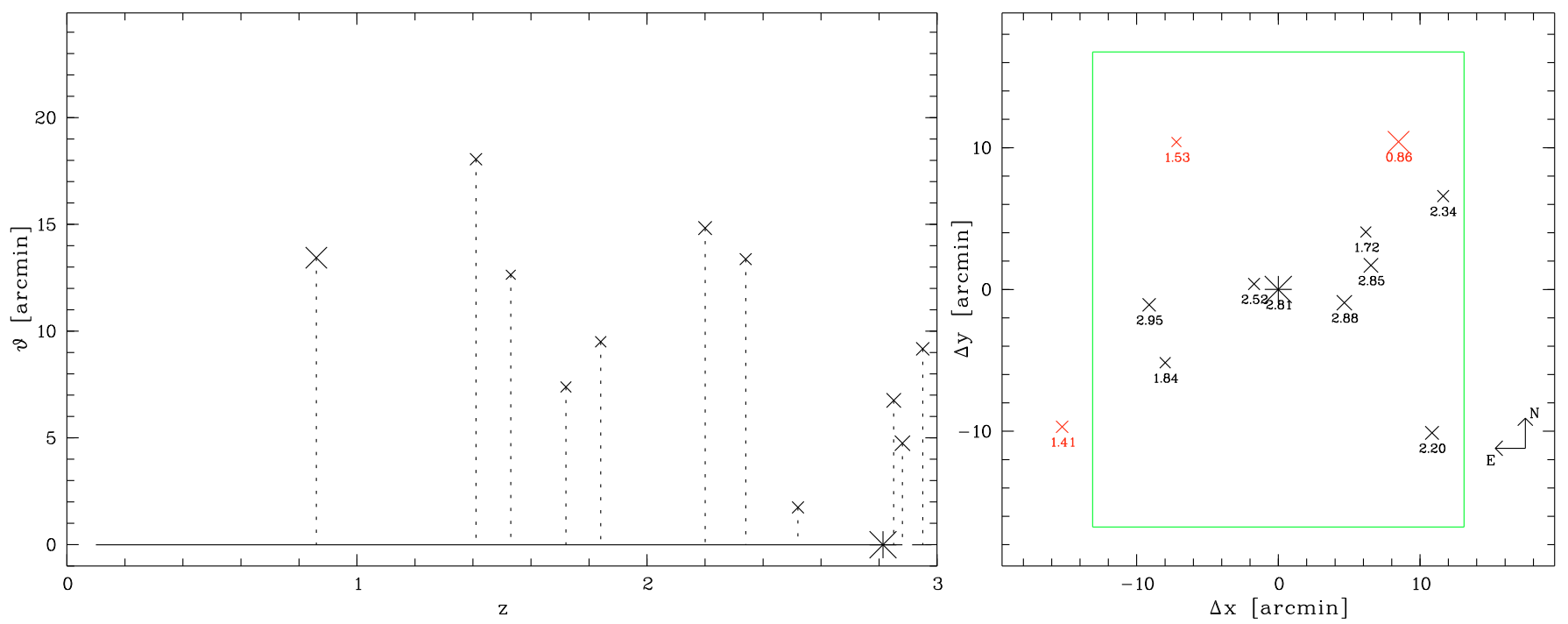

Fig. C. 8. As Fig. 15 for the field centred near PKS 0528-250.
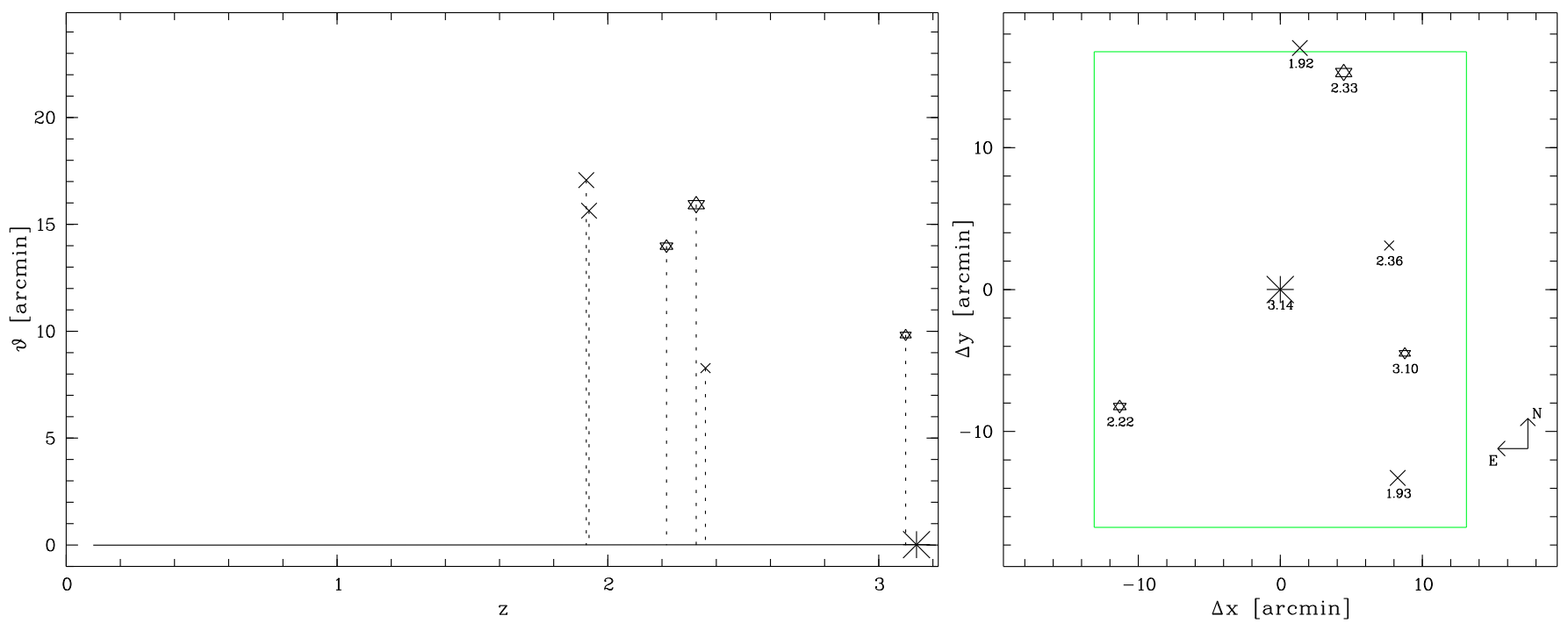

Fig. C. 9. As Fig. 15 for the field centred near CTQ 0460. 
G. Worseck et al.: A slitless spectroscopic survey for quasars near quasars, Online Material p 14
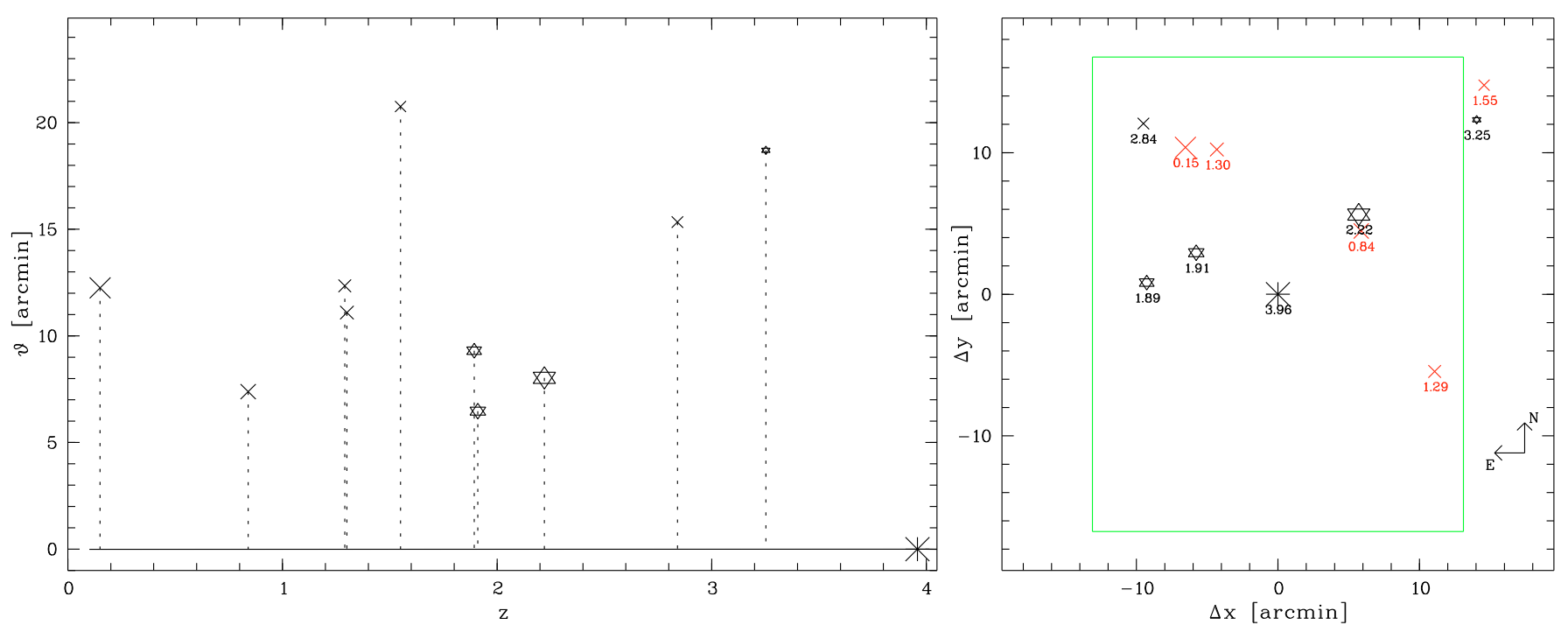

Fig. C. 10. As Fig. 15 for the field centred near BR 1117-1329.
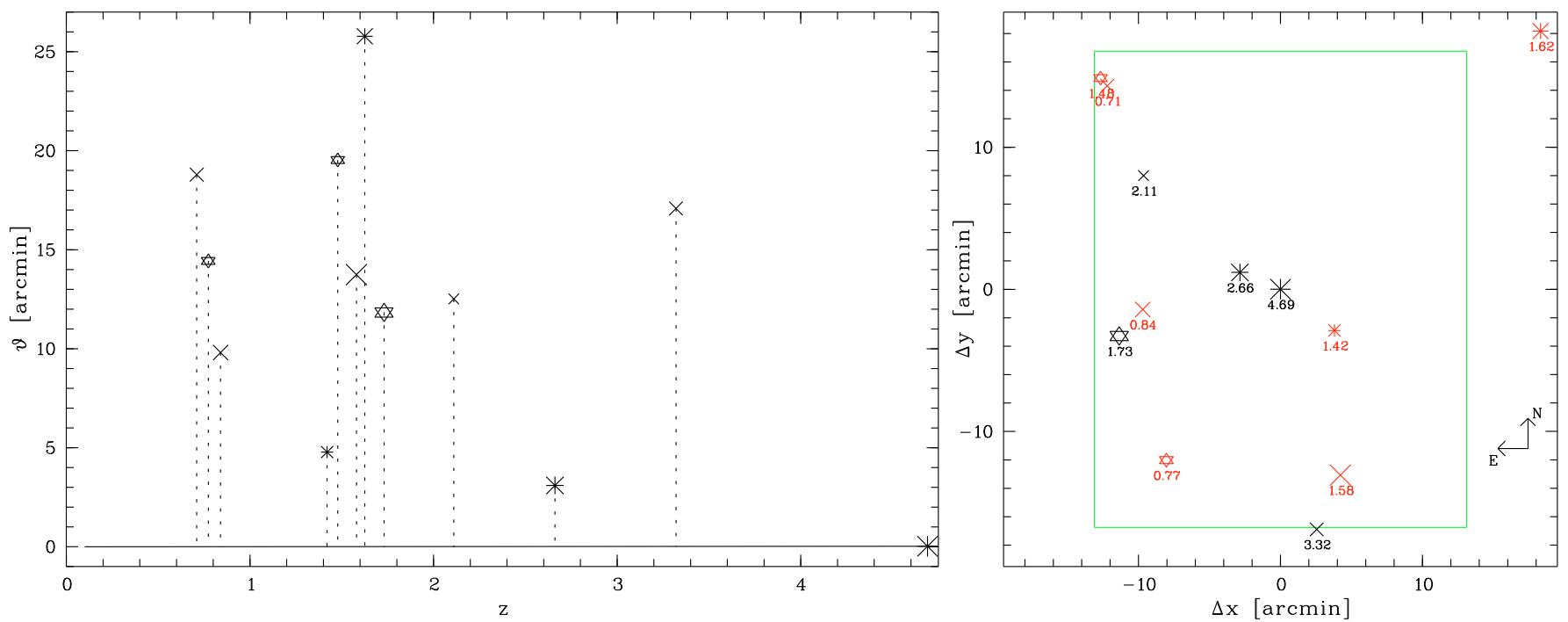

Fig. C. 11. As Fig. 15 for the field centred near BR 1202-0725.
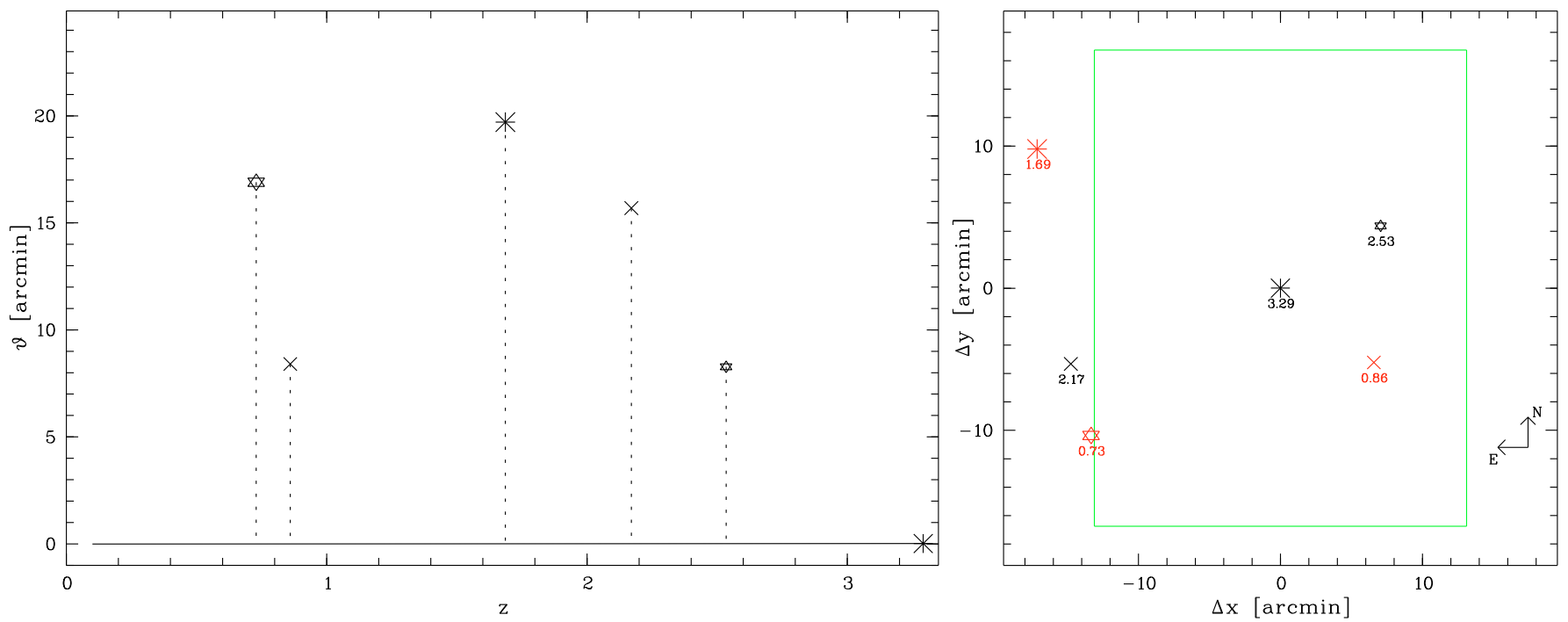

Fig. C. 12. As Fig. 15 for the field centred near Q 1209+093. 
G. Worseck et al.: A slitless spectroscopic survey for quasars near quasars, Online Material $p 15$
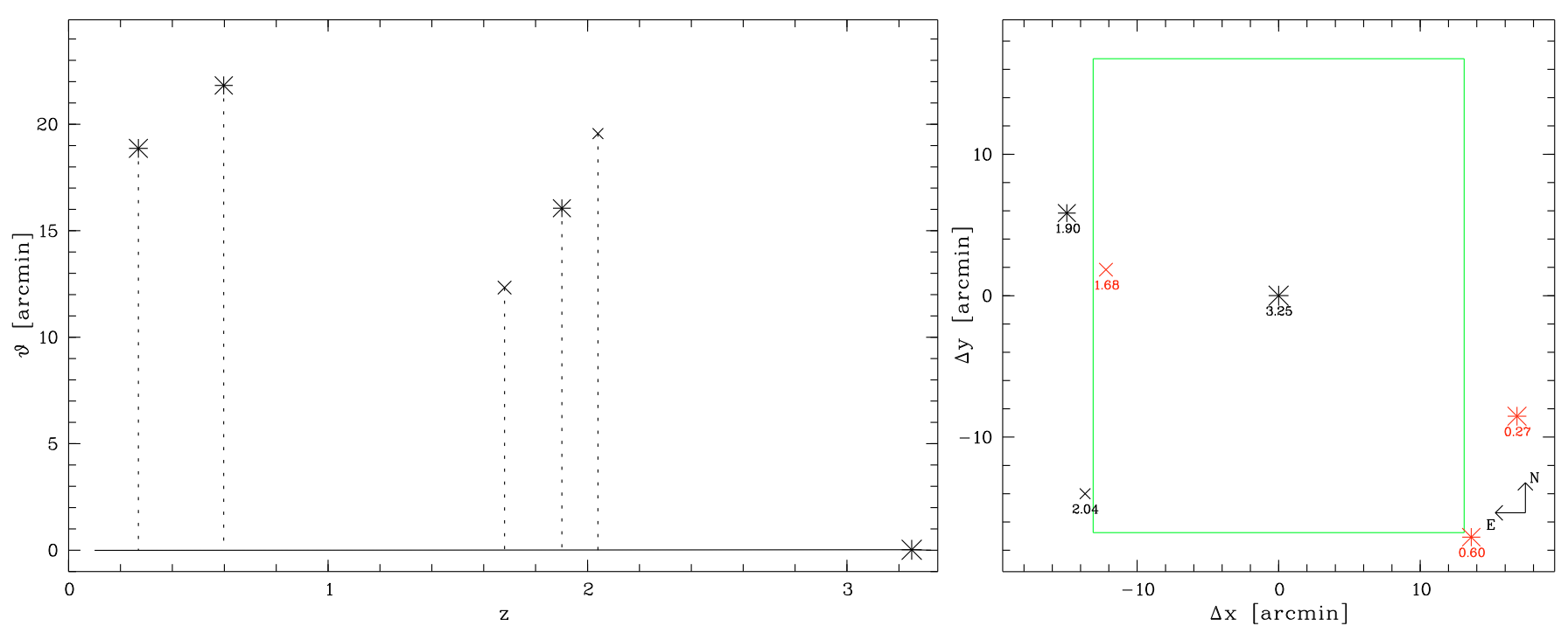

Fig. C. 13. As Fig. 15 for the field centred near Q 1451+123.
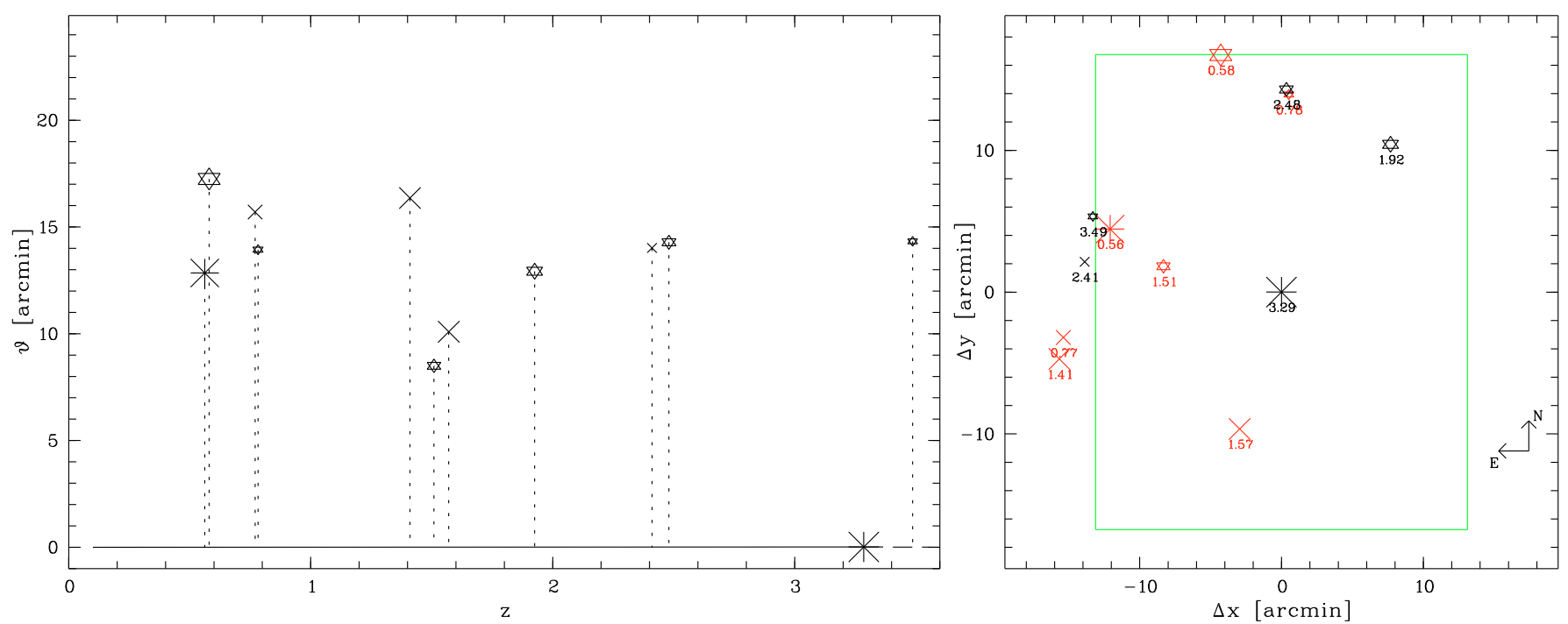

Fig. C. 14. As Fig. 15 for the field centred near PKS 2126-158.
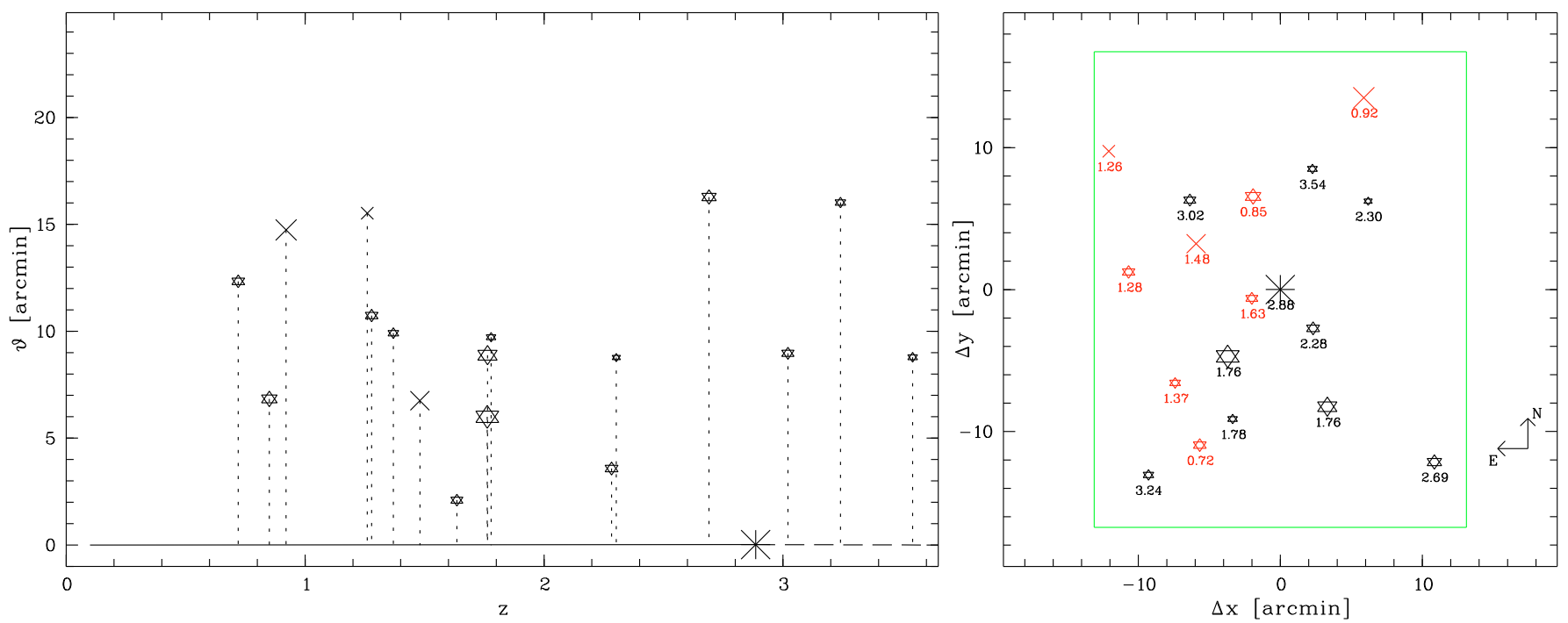

Fig. C. 15. As Fig. 15 for the field centred near HE 2347-4342. 
G. Worseck et al.: A slitless spectroscopic survey for quasars near quasars, Online Material p 16

Table C. 1. List of confirmed $z \geq 1.7$ quasars and AGN within a radius $<30^{\prime}$ around the central quasars in the survey fields. The columns list the name of the field, the name of the confirmed quasar, its right ascension and declination, its redshift, its optical magnitude with filter, the angular distance to the central quasar and the discovery paper.

\begin{tabular}{|c|c|c|c|c|c|c|c|c|}
\hline Field & QSO & $\alpha(\mathrm{J} 2000)$ & $\delta(\mathrm{J} 2000)$ & $z$ & Magnitude & Filter & $\vartheta\left[{ }^{\prime}\right]$ & Discovery paper \\
\hline \multirow[t]{9}{*}{ Q 0000-263 } & QNQ J00040-2603 & $00^{\mathrm{h}} 04^{\mathrm{m}} 05^{\mathrm{s}} .33$ & $-26^{\circ} 03^{\prime} 41^{\prime \prime} 9$ & 2.002 & 20.54 & $B$ & 9.54 & this paper \\
\hline & QNQ J00035-2610 & $00^{\mathrm{h}} 03^{\mathrm{m}} 31^{\mathrm{s}} .88$ & $-26^{\circ} 10^{\prime} 54^{\prime \prime} 8$ & 2.771 & 21.61 & $B$ & 7.90 & this paper \\
\hline & QNQ J00028-2547 & $00^{\mathrm{h}} 02^{\mathrm{m}} 53.86$ & $-25^{\circ} 47^{\prime} 43^{\prime \prime}{ }^{\prime} 1$ & 2.812 & 19.84 & $B$ & 16.87 & this paper \\
\hline & QNQ J00035-2551 & $00^{\mathrm{h}} 03^{\mathrm{m}} 33^{\mathrm{s}} .84$ & $-25^{\circ} 51^{\prime} 49^{\prime \prime} 8$ & 2.875 & 20.99 & $B$ & 11.70 & this paper \\
\hline & Q $0000-\mathrm{C} 14$ & $00^{\mathrm{h}} 03^{\mathrm{m}} 30.39$ & $-26^{\circ} 01^{\prime} 20^{\prime \prime} 7$ & 3.057 & 25.33 & $g$ & 2.56 & Steidel et al. (2003) \\
\hline & QNQ J00038-2617 & $00^{\mathrm{h}} 03^{\mathrm{m}} 51^{\mathrm{s}} .44$ & $-26^{\circ} 17^{\prime} 37^{\prime \prime} 8$ & 3.073 & 22.09 & $B$ & 15.72 & this paper \\
\hline & $\mathrm{Q} 0000-\mathrm{C} 7$ & $00^{\mathrm{h}} 03^{\mathrm{m}} 28.85$ & $-26^{\circ} 03^{\prime} 53^{\prime \prime} 3$ & 3.426 & 24.28 & $g$ & 1.47 & Steidel et al. (2003) \\
\hline & Q 0000-C5 & $00^{\mathrm{h}} 03^{\mathrm{m}} 28^{\mathrm{s}} .94$ & $-26^{\circ} 05^{\prime} 26^{\prime \prime} 1$ & 3.791 & 24.70 & $g$ & 2.55 & Steidel et al. (2003) \\
\hline & Q $0000-263$ & $00^{\mathrm{h}} 03^{\mathrm{m}} 22^{\mathrm{s}} .91$ & $-26^{\circ} 03^{\prime} 16^{\prime \prime} 3$ & 4.125 & 17.53 & $R$ & 0.00 & Shaver (1987) \\
\hline \multirow[t]{5}{*}{ Q $0002-422$} & QNQ J00041-4158 & $00^{\mathrm{h}} 04^{\mathrm{m}} 09^{\mathrm{s}} .01$ & $-41^{\circ} 58^{\prime} 32^{\prime \prime} 4$ & 1.720 & 20.36 & $B$ & 7.34 & this paper \\
\hline & QNQ J00045-4201 & $00^{\mathrm{h}} 04^{\mathrm{m}} 32^{\mathrm{s}} .75$ & $-42^{\circ} 01^{\prime} 33^{\prime \prime} \cdot 9$ & 2.157 & 20.35 & $B$ & 4.98 & this paper \\
\hline & Q $0000-4239$ & $00^{\mathrm{h}} 03^{\mathrm{m}} 29^{\mathrm{s}} .20$ & $-42^{\circ} 22^{\prime} 32^{\prime \prime} 0$ & 2.190 & 21.10 & $V$ & 29.01 & Osmer (1980) \\
\hline & Q $0001-4227$ & $00^{\mathrm{h}} 03^{\mathrm{m}} 50^{\mathrm{s}} \cdot 90$ & $-42^{\circ} 11^{\prime} 07^{\prime \prime} 8$ & 2.260 & 19.80 & $B$ & 17.29 & Osmer (1980) \\
\hline & Q 0002-422 & $00^{\mathrm{h}} 04^{\mathrm{m}} 48^{\mathrm{s}} .11$ & $-41^{\circ} 57^{\prime} 28^{\prime \prime} 8$ & 2.767 & 17.20 & V & 0.00 & Osmer \& Smith (1976) \\
\hline \multirow[t]{7}{*}{ Q 0055-269 } & Q $0053-2656$ & $00^{\mathrm{h}} 55^{\mathrm{m}} 44^{\mathrm{s}} .90$ & $-26^{\circ} 40^{\prime} 31^{\prime \prime} .^{\prime}$ & 1.882 & 19.67 & $V$ & 29.83 & La Franca et al. (1999) \\
\hline & QNQ J00576-2626 & $00^{\mathrm{h}} 57^{\mathrm{m}} 36^{\mathrm{s}} .26$ & $-26^{\circ} 26^{\prime} 57^{\prime \prime} 11$ & 1.942 & 20.73 & $B$ & 16.99 & this paper \\
\hline & Q $0056-2700$ & $00^{\mathrm{h}} 59^{\mathrm{m}} 00^{\mathrm{s}} .69$ & $-26^{\circ} 44^{\prime} 36^{\prime \prime} 0$ & 2.294 & 20.55 & $B$ & 14.08 & La Franca et al. (1999) \\
\hline & Q $0055-2654$ & $00^{\mathrm{h}} 58^{\mathrm{m}} 11^{\mathrm{s}} .11$ & $-26^{\circ} 38^{\prime} 00^{\prime \prime} 4$ & 2.389 & 20.46 & $B$ & 6.00 & La Franca et al. (1999) \\
\hline & QNQ J00582-2649 & $00^{\mathrm{h}} 58^{\mathrm{m}} 13.94$ & $-26^{\circ} 49^{\prime} 19^{\prime \prime} .2$ & 2.572 & 22.02 & $B$ & 7.06 & this paper \\
\hline & QNQ J00583-2626 & $00^{\mathrm{h}} 58^{\mathrm{m}} 19.50$ & $-26^{\circ} 26^{\prime} 12^{\prime \prime} 8$ & 2.720 & 21.00 & $B$ & 17.69 & this paper \\
\hline & Q $0055-269$ & $00^{\mathrm{h}} 57^{\mathrm{m}} 57^{\mathrm{s}} .92$ & $-26^{\circ} 43^{\prime} 14^{\prime \prime} \cdot 2$ & 3.665 & 17.50 & $V$ & 0.00 & Hazard \& McMahon (1985) \\
\hline \multirow[t]{11}{*}{ Q $0302-003$} & SDSS J0305+0007 & $03^{\mathrm{h}} 05^{\mathrm{m}} 20.89$ & $+00^{\circ} 07^{\prime} 33^{\prime \prime} 4$ & 1.758 & 20.71 & $r$ & 17.58 & Schneider et al. (2007) \\
\hline & QSO $03022-0023$ & $03^{\mathrm{h}} 04^{\mathrm{m}} 45^{\mathrm{s}} .94$ & $-00^{\circ} 11^{\prime} 38^{\prime \prime} 2$ & 2.142 & 22.46 & $V$ & 3.55 & Jakobsen et al. (2003) \\
\hline & QNQ J03052-0016 & $03^{\mathrm{h}} 05^{\mathrm{m}} 15^{\mathrm{s}} .62$ & $-00^{\circ} 16^{\prime} 14^{\prime \prime} 4$ & 2.290 & 20.05 & $B$ & 10.28 & Worseck \& Wisotzki (2006) \\
\hline & SDSS J0303-0020B & $03^{\mathrm{h}} 03^{\mathrm{m}} 35^{\mathrm{s}} .42$ & $-00^{\circ} 20^{\prime} 01^{\prime \prime} 1$ & 2.718 & 19.67 & $r$ & 22.03 & Hennawi et al. (2006a) \\
\hline & QNQ J03052+0000 & $03^{\mathrm{h}} 05^{\mathrm{m}} 16^{\mathrm{s}} .95$ & $+00^{\circ} 00^{\prime} 43^{\prime \prime} 5$ & 2.808 & 21.79 & $B$ & 11.22 & Worseck \& Wisotzki (2006) \\
\hline & Q $0302-D 113$ & $03^{\mathrm{h}} 04^{\mathrm{m}} 30^{\mathrm{s}} .33$ & $-00^{\circ} 08^{\prime} 11^{\prime \prime} 4$ & 2.920 & 24.68 & $g$ & 4.88 & Steidel et al. (2003) \\
\hline & QSO 03020-0014 & $03^{\mathrm{h}} 04^{\mathrm{m}} 35^{\mathrm{s}} .37$ & $-00^{\circ} 02^{\prime} 500^{\prime} 9$ & 3.050 & 20.44 & V & 6.48 & Jakobsen et al. (2003) \\
\hline & Q 0301-005 & $03^{\mathrm{h}} 03^{\mathrm{m}} 41^{\mathrm{s}} .05$ & $-00^{\circ} 23^{\prime} 21^{\prime \prime} .8$ & 3.231 & 17.64 & $r$ & 22.92 & Barbieri \& Cristiani (1986) \\
\hline & Q $0302-003$ & $03^{\mathrm{h}} 04^{\mathrm{m}} 49.86$ & $-00^{\circ} 08^{\prime} 13^{\prime \prime} 4$ & 3.285 & 18.76 & $B$ & 0.00 & Barbieri \& Cristiani (1986) \\
\hline & SDSS J0305-0006 & $03^{\mathrm{h}} 05^{\mathrm{m}} 05^{\mathrm{s}} .90$ & $-00^{\circ} 06^{\prime} 16^{\prime \prime} 5$ & 3.450 & 20.80 & $r$ & 4.46 & Schneider et al. (2007) \\
\hline & SDSS J0303-0019 & $03^{\mathrm{h}} 03^{\mathrm{m}} 31^{\mathrm{s}} .40$ & $-00^{\circ} 19^{\prime} 12^{\prime \prime} 9$ & 6.070 & 23.92 & $i$ & 22.48 & Jiang et al. (2008) \\
\hline \multirow[t]{10}{*}{ Q $0347-383$} & QNQ J03500-3820 & $03^{\mathrm{h}} 50^{\mathrm{m}} 04^{\mathrm{s}} .26$ & $-38^{\circ} 20^{\prime} 51^{\prime \prime} 2$ & 1.819 & 20.03 & $B$ & 11.09 & this paper \\
\hline & QNQ J03490-3812 & $03^{\mathrm{h}} 49^{\mathrm{m}} 03^{\mathrm{s}} .60$ & $-38^{\circ} 12^{\prime} 35^{\prime \prime} .8$ & 1.945 & 21.66 & $B$ & 8.14 & this paper \\
\hline & QNQ J03496-3821 & $03^{\mathrm{h}} 49^{\mathrm{m}} 39^{\mathrm{s}} .31$ & $-38^{\circ} 21^{\prime} 34^{\prime \prime} 1$ & 2.351 & 19.43 & $B$ & 11.08 & this paper \\
\hline & QNQ J03496-3810 & $03^{\mathrm{h}} 49^{\mathrm{m}} 36^{\mathrm{s}} .28$ & $-38^{\circ} 10^{\prime} 02^{\prime \prime} 1$ & 2.433 & 20.87 & $B$ & 1.53 & this paper \\
\hline & QNQ J03495-3806 & $03^{\mathrm{h}} 49^{\mathrm{m}} 32^{\mathrm{s}} .61$ & $-38^{\circ} 06^{\prime} 45^{\prime \prime} 1$ & 2.475 & 20.86 & $B$ & 4.35 & this paper \\
\hline & QNQ J03508-3812 & $03^{\mathrm{h}} 50^{\mathrm{m}} 50^{\mathrm{s}} .70$ & $-38^{\circ} 12^{\prime} 39^{\prime \prime} 0$ & 2.705 & 20.86 & $B$ & 13.34 & this paper \\
\hline & QNQ J03503-3800 & $03^{\mathrm{h}} 50^{\mathrm{m}} 20^{\mathrm{s}} .01$ & $-38^{\circ} 00^{\prime} 03^{\prime \prime} 6$ & 2.734 & 20.67 & $B$ & 12.67 & this paper \\
\hline & QNQ J03490-3825 & $03^{\mathrm{h}} 49^{\mathrm{m}} 02^{\mathrm{s}} .58$ & $-38^{\circ} 25^{\prime} 21^{\prime \prime} 5$ & 2.777 & 20.52 & $B$ & 16.89 & this paper \\
\hline & QNQ J03494-3826 & $03^{\mathrm{h}} 49^{\mathrm{m}} 28^{\mathrm{s}} .40$ & $-38^{\circ} 26^{\prime} 11^{\prime \prime} 6$ & 2.782 & 20.97 & $B$ & 15.96 & this paper \\
\hline & Q 0347-383 & $03^{\mathrm{h}} 49^{\mathrm{m}} 43^{\mathrm{s}} .68$ & $-38^{\circ} 10^{\prime} 31^{\prime \prime} .3$ & 3.220 & 18.66 & $B$ & 0.00 & Osmer \& Smith (1980) \\
\hline \multirow[t]{4}{*}{ CTQ 0247} & QNQ J04061-4401 & $04^{\mathrm{h}} 06^{\mathrm{m}} 10^{\mathrm{s}} .38$ & $-44^{\circ} 01^{\prime} 00^{\prime} 0$ & 2.410 & 19.35 & $B$ & 15.25 & this paper \\
\hline & CTQ 0247 & $04^{\mathrm{h}} 07^{\mathrm{m}} 17^{\mathrm{s}} .99$ & $-44^{\circ} 10^{\prime} 13^{\prime \prime} 4$ & 3.025 & 17.40 & V & 0.00 & Maza et al. (1993) \\
\hline & QNQ J04075-4416 & $04^{\mathrm{h}} 07^{\mathrm{m}} 35^{\mathrm{s}} .34$ & $-44^{\circ} 16^{\prime} 04^{\prime \prime} 1$ & 3.034 & 21.75 & $B$ & 6.62 & this paper \\
\hline & QNQ J04084-4420 & $04^{\mathrm{h}} 08^{\mathrm{m}} 29^{\mathrm{s}} .02$ & $-44^{\circ} 20^{\prime} 14^{\prime \prime} 4$ & 3.080 & 22.88 & $B$ & 16.19 & this paper \\
\hline \multirow[t]{10}{*}{ Q 0420-388 } & MS $0420-3838 B$ & $04^{\mathrm{h}} 21^{\mathrm{m}} 50^{\mathrm{s}} \cdot 10$ & $-38^{\circ} 31^{\prime} 14^{\prime \prime} 0$ & 1.931 & 20.61 & $B$ & 14.48 & Ciliegi et al. (1994) \\
\hline & QNQ J04229-3831 & $04^{\mathrm{h}} 22^{\mathrm{m}} 59^{\mathrm{s}} .97$ & $-38^{\circ} 31^{\prime} 37^{\prime \prime} 1$ & 1.990 & 19.98 & $B$ & 15.93 & this paper \\
\hline & QNQ J04222-3829 & $04^{\mathrm{h}} 22^{\mathrm{m}} 17^{\mathrm{s}} .31$ & $-38^{\circ} 29^{\prime} 33^{\prime \prime} .4$ & 2.168 & 20.91 & $B$ & 15.33 & this paper \\
\hline & QNQ J04215-3857 & $04^{\mathrm{h}} 21^{\mathrm{m}} 34^{\mathrm{s}} .81$ & $-38^{\circ} 57^{\prime} 03^{\prime \prime} 4$ & 2.235 & 22.23 & $B$ & 14.45 & this paper \\
\hline & QNQ J04215-3854 & $04^{\mathrm{h}} 21^{\mathrm{m}} 32^{\mathrm{s}} .16$ & $-38^{\circ} 54^{\prime} 50^{\prime \prime} 6$ & 2.270 & 19.49 & $B$ & 12.97 & this paper \\
\hline & PKS 0422-389 & $04^{\mathrm{h}} 24^{\mathrm{m}} 33^{\mathrm{s}} .80$ & $-38^{\circ} 48^{\prime} 42^{\prime \prime} 0$ & 2.346 & 18.00 & V & 27.36 & Hook et al. (2003) \\
\hline & Q $0420-3850$ & $04^{\mathrm{h}} 22^{\mathrm{m}} 21^{\mathrm{s}} .54$ & $-38^{\circ} 43^{\prime} 12^{\prime \prime} 1$ & 2.410 & 21.01 & $B$ & 2.13 & Hewitt \& Burbidge (1993) \\
\hline & QNQ J04212-3853 & $04^{\mathrm{h}} 21^{\mathrm{m}} 12^{\mathrm{s}} .49$ & $-38^{\circ} 53^{\prime} 28^{\prime \prime} .5$ & 2.723 & 20.08 & $B$ & 14.87 & this paper \\
\hline & QNQ J04230-3853 & $04^{\mathrm{h}} 23^{\mathrm{m}} 01^{\mathrm{s}} .12$ & $-38^{\circ} 53^{\prime} 14^{\prime \prime} 5$ & 3.042 & 21.95 & $B$ & 12.30 & this paper \\
\hline & Q $0420-388$ & $04^{\mathrm{h}} 22^{\mathrm{m}} 14^{\mathrm{s}} .81$ & $-38^{\circ} 44^{\prime} 52^{\prime \prime} 9$ & 3.120 & 16.90 & V & 0.00 & Osmer \& Smith (1980) \\
\hline PKS $0528-250$ & PKS 0528-250 & $05^{\mathrm{h}} 30^{\mathrm{m}} 07^{\mathrm{s}} .96$ & $-25^{\circ} 03^{\prime} 29^{\prime \prime} 9$ & 2.813 & 17.50 & $V$ & 0.00 & Wright et al. (1977) \\
\hline HE $0940-1050$ & QNQ J09430-1108 & $09^{\mathrm{h}} 43^{\mathrm{m}} 00^{\mathrm{s}} .27$ & $-11^{\circ} 08^{\prime} 06^{\prime \prime} 3$ & 1.730 & 21.03 & $B$ & 4.05 & this paper \\
\hline & QNQ J09424-1047 & $09^{\mathrm{h}} 42^{\mathrm{m}} 24^{\mathrm{s}} .17$ & $-10^{\circ} 47^{\prime} 58^{\prime \prime} .5$ & 1.971 & 20.61 & $B$ & 17.94 & this paper \\
\hline & QNQ J09437-1057 & $09^{\mathrm{h}} 43^{\mathrm{m}} 45^{\mathrm{s}} .15$ & $-10^{\circ} 57^{\prime} 58^{\prime \prime} 8$ & 2.023 & 20.64 & $B$ & 14.24 & this paper \\
\hline & QNQ J09435-1049 & $09^{\mathrm{h}} 43^{\mathrm{m}} 30^{\mathrm{s}} .04$ & $-10^{\circ} 49^{\prime} 58^{\prime \prime} 9$ & 2.216 & 20.79 & $B$ & 17.01 & this paper \\
\hline & QNQ J09425-1048 & $09^{\mathrm{h}} 42^{\mathrm{m}} 30^{\mathrm{s}} .58$ & $-10^{\circ} 48^{\prime} 50^{\prime \prime} 8$ & 2.325 & 19.80 & $B$ & 16.55 & this paper \\
\hline & QNQ J09434-1053 & $09^{\mathrm{h}} 43^{\mathrm{m}} 24^{\mathrm{s}} .21$ & $-10^{\circ} 53^{\prime} 32^{\prime \prime} 9$ & 2.760 & 21.16 & $B$ & 13.24 & this paper \\
\hline & QNQ J09427-1121 & $09^{\mathrm{h}} 42^{\mathrm{m}} 44^{\mathrm{s}} .42$ & $-11^{\circ} 21^{\prime} 38^{\prime \prime} 9$ & 2.963 & 20.99 & $B$ & 17.37 & this paper \\
\hline & QNQ J09437-1052 & $09^{\mathrm{h}} 43^{\mathrm{m}} 42^{\mathrm{s}} .99$ & $-10^{\circ} 52^{\prime} 31^{\prime \prime} 7$ & 3.018 & 20.78 & $B$ & 17.01 & this paper \\
\hline & HE 0940-1050 & $09^{\mathrm{h}} 42^{\mathrm{m}} 53^{\mathrm{s}} .40$ & $-11^{\circ} 04^{\prime} 25^{\prime \prime} 0$ & 3.088 & 16.96 & $B_{J}$ & 0.00 & Reimers et al. (1995) \\
\hline CTQ 0460 & QNQ J10399-2321 & $10^{\mathrm{h}} 39^{\mathrm{m}} 58^{\mathrm{s}} .77$ & $-23^{\circ} 21^{\prime} 40^{\prime \prime} 3$ & 2.216 & 20.73 & $B$ & 14.00 & this paper \\
\hline & QNQ J10388-2258 & $10^{\mathrm{h}} 38^{\mathrm{m}} 50^{\mathrm{s}} .12$ & $-22^{\circ} 58^{\prime} 08^{\prime \prime} 9$ & 2.326 & 19.63 & $B$ & 15.92 & this paper \\
\hline & QNQ J10385-2317 & $10^{\mathrm{h}} 38^{\mathrm{m}} 31^{\mathrm{s}} .54$ & $-23^{\circ} 17^{\prime} 55^{\prime \prime} 3$ & 3.099 & 21.28 & $B$ & 9.81 & this paper \\
\hline & CTQ 0460 & $10^{\mathrm{h}} 39^{\mathrm{m}} 09^{\mathrm{s}} .51$ & $-23^{\circ} 13^{\prime} 25^{\prime \prime} 7$ & 3.139 & 17.50 & V & 0.00 & Maza et al. (1995) \\
\hline BR $1117-1329$ & QNQ J11208-1345 & $11^{\mathrm{h}} 20^{\mathrm{m}} 48^{\mathrm{s}} .50$ & $-13^{\circ} 45^{\prime} 35^{\prime \prime} 6$ & 1.893 & 20.10 & $B$ & 9.31 & this paper \\
\hline & QNQ J11205-1343 & $11^{\mathrm{h}} 20^{\mathrm{m}} 34^{\mathrm{s}} .09$ & $-13^{\circ} 43^{\prime} 28^{\prime \prime} 9$ & 1.910 & 19.84 & $B$ & 6.48 & this paper \\
\hline & QNQ J11197-1340 & $11^{\mathrm{h}} 19^{\mathrm{m}} 46^{\mathrm{s}} .71$ & $-13^{\circ} 40^{\prime} 47^{\prime \prime} 11$ & 2.220 & 18.32 & $B$ & 8.03 & this paper \\
\hline & QNQ J11192-1334 & $11^{\mathrm{h}} 19^{\mathrm{m}} 12^{\mathrm{s}} .37$ & $-13^{\circ} 34^{\prime} 05^{\prime \prime} 5$ & 3.252 & 22.55 & $B$ & 18.71 & this paper \\
\hline & BR $1117-1329$ & $11^{\mathrm{h}} 20^{\mathrm{m}} 10^{\mathrm{s}} 30$ & $-13^{\circ} 46^{\prime} 25^{\prime \prime} 0$ & 3.958 & 18.00 & $R$ & 0.00 & Storrie-Lombardi et al. (1996) \\
\hline
\end{tabular}


G. Worseck et al.: A slitless spectroscopic survey for quasars near quasars, Online Material $p 17$

Table C. 1. continued.

\begin{tabular}{|c|c|c|c|c|c|c|c|c|}
\hline Field & QSO & $\alpha(\mathrm{J} 2000)$ & $\delta(\mathrm{J} 2000)$ & $z$ & Magnitude & Filter & $\vartheta\left[{ }^{\prime}\right]$ & Discovery paper \\
\hline \multirow[t]{3}{*}{ BR $1202-0725$} & QNQ J12061-0745 & $12^{\mathrm{h}} 06^{\mathrm{m}} 08^{\mathrm{s}} .91$ & $-07^{\circ} 45^{\prime} 49^{\prime \prime} 8$ & 1.730 & 19.15 & $B$ & 11.81 & this paper \\
\hline & CIRSI 05 & $12^{\mathrm{h}} 05^{\mathrm{m}} 34^{\mathrm{s}} .59$ & $-07^{\circ} 41^{\prime} 21^{\prime \prime} 4$ & 2.661 & 19.39 & $B$ & 3.08 & Sharp et al. (2002) \\
\hline & BR $1202-0725$ & $12^{\mathrm{h}} 05^{\mathrm{m}} 23^{\mathrm{s}} .12$ & $-07^{\circ} 42^{\prime} 32^{\prime \prime} 5$ & 4.690 & 18.70 & $R$ & 0.00 & McMahon et al. (1994) \\
\hline \multirow{2}{*}{ Q 1209+093 } & QNQ J12111+0906 & $12^{\mathrm{h}} 11^{\mathrm{m}} 06^{\mathrm{s}} .59$ & $+09^{\circ} 06^{\prime} 43^{\prime \prime} 6$ & 2.534 & 21.17 & $B$ & 8.26 & this paper \\
\hline & Q $1209+093$ & $12^{\mathrm{h}} 11^{\mathrm{m}} 34^{\mathrm{s}} .95$ & $+09^{\circ} 02^{\prime} 20^{\prime} 9$ & 3.291 & 19.04 & $g$ & 0.00 & Hazard et al. (1987) \\
\hline \multirow{3}{*}{ Q $1451+123$} & SDSS J1455+1216 & $14^{\mathrm{h}} 55^{\mathrm{m}} 19^{\mathrm{s}} .92$ & $+12^{\circ} 16^{\prime} 45^{\prime \prime} 1$ & 1.901 & 19.29 & $g$ & 16.08 & Schneider et al. (2007) \\
\hline & Q $1451+123$ & $14^{\mathrm{h}} 54^{\mathrm{m}} 18^{\mathrm{s}} \cdot 61$ & $+12^{\circ} 10^{\prime} 54^{\prime \prime} 8$ & 3.249 & 18.80 & $V$ & 0.00 & Hazard et al. (1986) \\
\hline & SDSS J1452+1156 & $14^{\mathrm{h}} 52^{\mathrm{m}} 47^{\mathrm{s}} .72$ & $+11^{\circ} 56^{\prime} 20^{\prime} .1$ & 4.026 & 21.44 & $g$ & 26.58 & Schneider et al. (2007) \\
\hline \multirow[t]{4}{*}{ PKS 2126-158 } & QNQ J21286-1528 & $21^{\mathrm{h}} 28^{\mathrm{m}} 40^{\mathrm{s}} .43$ & $-15^{\circ} 28^{\prime} 15^{\prime \prime} 7$ & 1.925 & 19.79 & $B$ & 12.93 & this paper \\
\hline & QNQ J21291-1524B & $21^{\mathrm{h}} 29^{\mathrm{m}} 10^{\mathrm{s}} 85$ & $-15^{\circ} 24^{\prime} 23^{\prime \prime} 7$ & 2.480 & 20.33 & $B$ & 14.29 & this paper \\
\hline & PKS 2126-158 & $21^{\mathrm{h}} 29^{\mathrm{m}} 12^{\mathrm{s}} .18$ & $-15^{\circ} 38^{\prime} 41^{\prime \prime} 0$ & 3.285 & 17.00 & $V$ & 0.00 & Jauncey et al. (1978) \\
\hline & QNQ J21301-1533 & $21^{\mathrm{h}} 30^{\mathrm{m}} 07^{\mathrm{s}} .46$ & $-15^{\circ} 33^{\prime} 20^{\prime} 9$ & 3.487 & 21.94 & $B$ & 14.34 & this paper \\
\hline \multirow[t]{26}{*}{ Q 2139-4434 } & Q $2138-4416$ & $21^{\mathrm{h}} 42^{\mathrm{m}} 07^{\mathrm{s}} .89$ & $-44^{\circ} 03^{\prime} 09^{\prime} .8$ & 1.732 & 19.38 & $B$ & 17.42 & Hawkins (2000) \\
\hline & QSO J2142-4406 & $21^{\mathrm{h}} 42^{\mathrm{m}} 38^{\mathrm{s}} .71$ & $-44^{\circ} 06^{\prime} 39^{\prime} .^{\prime} 0$ & 1.736 & 21.10 & $B$ & 13.83 & Francis et al. (2004) \\
\hline & QSO J2143-4410 & $21^{\mathrm{h}} 43^{\mathrm{m}} 29^{\mathrm{s}} .62$ & $-44^{\circ} 10^{\prime} 11^{\prime \prime} 9$ & 1.768 & 21.71 & $B$ & 15.24 & Francis et al. (2004) \\
\hline & QSO J2142-4430 & $21^{\mathrm{h}} 42^{\mathrm{m}} 51^{\mathrm{s}} .50$ & $-44^{\circ} 30^{\prime} 43^{\prime \prime} .2$ & 1.795 & 20.26 & $B$ & 11.40 & Francis et al. (2004) \\
\hline & QSO J2142-4432 & $21^{\mathrm{h}} 42^{\mathrm{m}} 43^{\mathrm{s}} .93$ & $-44^{\circ} 32^{\prime} 50^{\prime} 8$ & 1.849 & 21.44 & $B$ & 12.97 & Francis et al. (2004) \\
\hline & QSO J2142-4358 & $21^{\mathrm{h}} 42^{\mathrm{m}} 29.03$ & $-43^{\circ} 58^{\prime} 38^{\prime \prime} 6$ & 1.853 & 21.96 & $B$ & 21.65 & Francis et al. (2004) \\
\hline & QSO J2140-4432 & $21^{\mathrm{h}} 40^{\mathrm{m}} 20^{\mathrm{s}} .82$ & $-44^{\circ} 32^{\prime} 53^{\prime \prime} 1$ & 1.863 & 20.69 & $B$ & 25.62 & Francis et al. (2004) \\
\hline & QSO J2142-4431 & $21^{\mathrm{h}} 42^{\mathrm{m}} 39^{\mathrm{s}} .38$ & $-44^{\circ} 31^{\prime} 15^{\prime \prime} 2$ & 2.036 & 22.79 & $B$ & 11.23 & Francis et al. (2004) \\
\hline & Q $2137-4422$ & $21^{\mathrm{h}} 40^{\mathrm{m}} 13.56$ & $-44^{\circ} 09^{\prime} 18^{\prime \prime} 4$ & 2.045 & 20.78 & $B$ & 26.11 & Hawkins (2000) \\
\hline & QSO J2140-4403 & $21^{\mathrm{h}} 40^{\mathrm{m}} 39^{\mathrm{s}} .08$ & $-44^{\circ} 03^{\prime} 07^{\prime \prime} 6$ & 2.097 & 20.31 & $B$ & 25.70 & Francis et al. (2004) \\
\hline & Q 2138-4420 & $21^{\mathrm{h}} 41^{\mathrm{m}} 23^{\mathrm{s}} 07$ & $-44^{\circ} 06^{\prime} 48^{\prime \prime} 0$ & 2.107 & 20.28 & $B$ & 17.56 & Hawkins (2000) \\
\hline & QSO J2144-4412 & $21^{\mathrm{h}} 44^{\mathrm{m}} 29^{\mathrm{s}} \cdot 09$ & $-44^{\circ} 12^{\prime} 03^{\prime \prime} 4$ & 2.140 & 22.86 & $B$ & 23.55 & Francis et al. (2004) \\
\hline & QSO J2144-4423 & $21^{\mathrm{h}} 44^{\mathrm{m}} 20^{\mathrm{s}} .92$ & $-44^{\circ} 23^{\prime} 49^{\prime \prime} 8$ & 2.162 & 20.49 & $B$ & 20.87 & Francis et al. (2004) \\
\hline & QSO J2143-4408 & $21^{\mathrm{h}} 43^{\mathrm{m}} 37^{\mathrm{s}} .66$ & $-44^{\circ} 08^{\prime} 01^{\prime \prime} 5$ & 2.166 & 22.02 & $B$ & 17.78 & Francis et al. (2004) \\
\hline & QSO J2141-4356 & $21^{\mathrm{h}} 41^{\mathrm{m}} 15^{\mathrm{s}} .43$ & $-43^{\circ} 56^{\prime} 00^{\prime} 7$ & 2.202 & 21.51 & $B$ & 27.36 & Francis et al. (2004) \\
\hline & QSO J2143-4356 & $21^{\mathrm{h}} 43^{\mathrm{m}} 23^{\mathrm{s}} .56$ & $-43^{\circ} 56^{\prime} 38^{\prime \prime} .6$ & 2.223 & 21.82 & $B$ & 25.81 & Francis et al. (2004) \\
\hline & QSO J2141-4442 & $21^{\mathrm{h}} 41^{\mathrm{m}} 54^{\mathrm{s}} .94$ & $-44^{\circ} 42^{\prime} 00^{\prime} 8$ & 2.224 & 21.89 & $B$ & 22.41 & Francis et al. (2004) \\
\hline & Q 2139-4444 & $21^{\mathrm{h}} 42^{\mathrm{m}} 31^{\mathrm{s}} .56$ & $-44^{\circ} 30^{\prime} 16^{\prime \prime} 5$ & 2.372 & 21.19 & $B$ & 10.04 & Hawkins (2000) \\
\hline & QSO J2142-4359 & $21^{\mathrm{h}} 42^{\mathrm{m}} 20^{\mathrm{s}} .67$ & $-43^{\circ} 59^{\prime} 20^{\prime} 0$ & 2.458 & 21.59 & $B$ & 20.97 & Francis et al. (2004) \\
\hline & QNQ J21434-4432 & $21^{\mathrm{h}} 43^{\mathrm{m}} 25^{\mathrm{s}} \cdot 26$ & $-44^{\circ} 32^{\prime} 11^{\prime \prime} 0$ & 2.709 & 19.33 & $B$ & 15.94 & this paper \\
\hline & QSO J2144-4407 & $21^{\mathrm{h}} 44^{\mathrm{m}} 17^{\mathrm{s}} .56$ & $-44^{\circ} 07^{\prime} 02^{\prime \prime} 0$ & 2.725 & 21.04 & $B$ & 24.01 & Francis et al. (2004) \\
\hline & QSO J2143-4435 & $21^{\mathrm{h}} 43^{\mathrm{m}} 23^{\mathrm{s}} 39$ & $-44^{\circ} 35^{\prime} 24^{\prime \prime} 0$ & 2.725 & 22.02 & $B$ & 18.27 & Francis et al. (2004) \\
\hline & Q $2138-4427$ & $21^{\mathrm{h}} 41^{\mathrm{m}} 59.79$ & $-44^{\circ} 13^{\prime} 25^{\prime \prime} .9$ & 3.120 & 18.13 & $V$ & 8.29 & Morris et al. (1991) \\
\hline & Q $2140-4406$ & $21^{\mathrm{h}} 43^{\mathrm{m}} 10^{\mathrm{s}} .50$ & $-43^{\circ} 52^{\prime} 55^{\prime \prime} .0$ & 3.180 & 20.64 & $V$ & 28.52 & Véron \& Hawkins (1995) \\
\hline & Q 2139-4434 & $21^{\mathrm{h}} 42^{\mathrm{m}} 25^{\mathrm{s}} .81$ & $-44^{\circ} 20^{\prime} 17^{\prime \prime} 2$ & 3.214 & 17.70 & $V$ & 0.00 & Morris et al. (1991) \\
\hline & Q $2139-4433$ & $21^{\mathrm{h}} 42^{\mathrm{m}} 22^{\mathrm{s}} \cdot 23$ & $-44^{\circ} 19^{\prime} 29^{\prime} 7$ & 3.228 & 21.63 & $B$ & 1.02 & Véron \& Hawkins (1995) \\
\hline \multirow[t]{7}{*}{ HE $2243-6031$} & QNQ J22454-6020 & $22^{\mathrm{h}} 45^{\mathrm{m}} 27^{\mathrm{s}} .22$ & $-60^{\circ} 20^{\prime} 25^{\prime \prime} .2$ & 1.984 & 20.94 & $B$ & 13.46 & this paper \\
\hline & QNQ J22455-6015 & $22^{\mathrm{h}} 45^{\mathrm{m}} 34.64$ & $-60^{\circ} 15^{\prime} 45^{\prime \prime} 9$ & 2.036 & 21.74 & $B$ & 11.71 & this paper \\
\hline & QNQ J22460-6024 & $22^{\mathrm{h}} 46^{\mathrm{m}} 01^{\mathrm{s}} .23$ & $-60^{\circ} 24^{\prime} 57^{\prime \prime} .5$ & 2.041 & 21.25 & $B$ & 12.46 & this paper \\
\hline & QNQ J22454-6011 & $22^{\mathrm{h}} 45^{\mathrm{m}} 29^{\mathrm{s}} .42$ & $-60^{\circ} 11^{\prime} 17^{\prime \prime} 1$ & 2.324 & 19.36 & $B$ & 13.16 & this paper \\
\hline & QNQ J22463-6009 & $22^{\mathrm{h}} 46^{\mathrm{m}} 18^{\mathrm{s}} 47$ & $-60^{\circ} 09^{\prime} 02^{\prime \prime} .5$ & 2.329 & 19.71 & $B$ & 9.20 & this paper \\
\hline & HE $2243-6031^{a}$ & $22^{\mathrm{h}} 47^{\mathrm{m}} 09^{\mathrm{s}} .10$ & $-60^{\circ} 15^{\prime} 45^{\prime \prime} 0$ & 3.010 & 16.40 & $V$ & 0.00 & \\
\hline & QNQ J22484-6002 & $22^{\mathrm{h}} 48^{\mathrm{m}} 29^{\mathrm{s}} .20$ & $-60^{\circ} 02^{\prime} 19^{\prime \prime} 4$ & 3.586 & 20.97 & $B$ & 16.72 & this paper \\
\hline \multirow[t]{10}{*}{ HE 2347-4342 } & QNQ J23509-4330 & $23^{\mathrm{h}} 50^{\mathrm{m}} 54^{\mathrm{s}} 80$ & $-43^{\circ} 30^{\prime} 42^{\prime \prime} .2$ & 1.762 & 18.23 & $B$ & 6.01 & Worseck et al. (2007) \\
\hline & QNQ J23502-4334 & $23^{\mathrm{h}} 50^{\mathrm{m}} 16^{\mathrm{s}} .18$ & $-43^{\circ} 34^{\prime} 14^{\prime \prime} 7$ & 1.763 & 18.95 & $B$ & 8.88 & Worseck et al. (2007) \\
\hline & QSO J23508-4335 & $23^{\mathrm{h}} 50^{\mathrm{m}} 52^{\mathrm{s}} .91$ & $-43^{\circ} 35^{\prime} 06^{\prime \prime} 8$ & 1.778 & 22.01 & $V$ & 9.73 & Worseck et al. (2007) \\
\hline & QNQ J23503-4328 & $23^{\mathrm{h}} 50^{\mathrm{m}} 21^{\mathrm{s}} .55$ & $-43^{\circ} 28^{\prime} 43^{\prime \prime} 7$ & 2.282 & 20.66 & $B$ & 3.57 & Worseck et al. (2007) \\
\hline & QSO J23500-4319 & $23^{\mathrm{h}} 50^{\mathrm{m}} 00^{\mathrm{s}} .28$ & $-43^{\circ} 19^{\prime} 46^{\prime \prime} 1$ & 2.302 & 22.61 & $V$ & 8.76 & Worseck et al. (2007) \\
\hline & QNQ J23495-4338 & $23^{\mathrm{h}} 49^{\mathrm{m}} 34^{\mathrm{s}} .53$ & $-43^{\circ} 38^{\prime} 08^{\prime \prime} 7$ & 2.690 & 20.21 & $B$ & 16.27 & Worseck et al. (2007) \\
\hline & HE 2347-4342 & $23^{\mathrm{h}} 50^{\mathrm{m}} 34^{\mathrm{s}} .21$ & $-43^{\circ} 25^{\prime} 59^{\prime} .^{\prime} 6$ & 2.885 & 17.18 & $B$ & 0.00 & Reimers et al. (1997) \\
\hline & QNQ J23511-4319 & $23^{\mathrm{h}} 51^{\mathrm{m}} 09^{\mathrm{s}} 44$ & $-43^{\circ} 19^{\prime} 41^{\prime \prime} 6$ & 3.020 & 21.00 & $B$ & 8.98 & Worseck et al. (2007) \\
\hline & QNQ J23514-4339 & $23^{\mathrm{h}} 51^{\mathrm{m}} 25^{\mathrm{s}} .54$ & $-43^{\circ} 39^{\prime} 02^{\prime \prime} .9$ & 3.240 & 21.57 & $B$ & 16.03 & Worseck et al. (2007) \\
\hline & QNQ J23503-4317 & $23^{\mathrm{h}} 50^{\mathrm{m}} 21^{\mathrm{s}} .94$ & $-43^{\circ} 17^{\prime} 30^{\prime} ._{0}$ & 3.542 & 21.94 & $B$ & 8.78 & Worseck et al. (2007) \\
\hline
\end{tabular}

${ }^{a}$ Both SIMBAD and NED list HE 2243-6031 at a wrong position. Here we give its coordinates measured on the DSS image, accurate to $\lesssim 1 "$. 
G. Worseck et al.: A slitless spectroscopic survey for quasars near quasars, Online Material p 18

Table 2. Observing log of the spectroscopic follow-up. The quasars reported in Papers I and II have been included for completeness. The columns list the quasar field, the name of the confirmed quasar, its right ascension and declination, its redshift with estimated error, its $B$ magnitude with $1 \sigma$ error, night of observation, the grism used, the exposure time, the seeing and the sky conditions during observation.

\begin{tabular}{|c|c|c|c|c|c|c|c|c|c|c|}
\hline Field & QSO & $\alpha(\mathrm{J} 2000)$ & $\delta(\mathbf{J} 2000)$ & $z$ & $B$ & Night & Grism & $t_{\exp }[\mathrm{s}]$ & Seeing & Transparency \\
\hline \multirow[t]{6}{*}{ Q 0000-263 } & QNQ J00025-2558 & $00^{\mathrm{h}} 02^{\mathrm{m}} 34^{\mathrm{s}} .83$ & $-25^{\circ} 58^{\prime} 44^{\prime \prime} .0$ & $0.887 \pm 0.002$ & $20.26 \pm 0.13$ & 18 Nov. 2004 & $300 \mathrm{~V}$ & 300 & 0.9 & clear \\
\hline & QNQ J00040-2603 & $00^{\mathrm{h}} 04^{\mathrm{m}} 05^{\mathrm{s}} .33$ & $-26^{\circ} 03^{\prime} 41^{\prime \prime} 9$ & $2.002 \pm 0.003$ & $20.54 \pm 0.16$ & 18 Nov. 2004 & $600 \mathrm{~B}$ & 1000 & 1.0 & clear \\
\hline & QNQ J00035-2610 & $00^{\mathrm{h}} 03^{\mathrm{m}} 31^{\mathrm{s}} .88$ & $-26^{\circ} 10^{\prime} 54^{\prime \prime} 8$ & $2.771 \pm 0.003$ & $21.61 \pm 0.42$ & 18 Nov. 2004 & $600 \mathrm{~B}$ & 1800 & 1.0 & clear \\
\hline & QNQ J00028-2547 & $00^{\mathrm{h}} 02^{\mathrm{m}} 53^{\mathrm{s}} .86$ & $-25^{\circ} 47^{\prime} 43^{\prime \prime} 1$ & $2.812 \pm 0.004$ & $19.84 \pm 0.10$ & 18 Nov. 2004 & $600 \mathrm{~B}$ & 400 & 0.8 & clear \\
\hline & QNQ J00035-2551 & $00^{\mathrm{h}} 03^{\mathrm{m}} 33.84$ & $-25^{\circ} 51^{\prime} 49^{\prime \prime} 8$ & $2.875 \pm 0.004$ & $20.99 \pm 0.25$ & 18 Nov. 2004 & $600 \mathrm{~B}$ & 1600 & $1 .{ }^{\prime \prime} 1$ & clear \\
\hline & QNQ J00038-2617 & $00^{\mathrm{h}} 03^{\mathrm{m}} 51^{\mathrm{s}} .44$ & $-26^{\circ} 17^{\prime} 37^{\prime \prime} 8$ & $3.073 \pm 0.003$ & $22.09 \pm 0.41$ & 18 Nov. 2004 & $300 \mathrm{~V}$ & 1200 & 1.0 & clear \\
\hline \multirow[t]{3}{*}{ Q 0002-422 } & QNQ J00043-4151 & $00^{\mathrm{h}} 04^{\mathrm{m}} 19^{\mathrm{s}} .05$ & $-41^{\circ} 51^{\prime} 10^{\prime} 6$ & $0.743 \pm 0.001$ & $19.84 \pm 0.16$ & 19 Nov. 2004 & $300 \mathrm{~V}$ & 300 & 1.3 & photometric \\
\hline & QNQ J00041-4158 & $00^{\mathrm{h}} 04^{\mathrm{m}} 09^{\mathrm{s}} .01$ & $-41^{\circ} 58^{\prime} 32^{\prime \prime} 4$ & $1.720 \pm 0.004$ & $20.36 \pm 0.23$ & 19 Nov. 2004 & $300 \mathrm{~V}$ & 400 & 1.3 & photometric \\
\hline & QNQ J00045-4201 & $00^{\mathrm{h}} 04^{\mathrm{m}} 32^{\mathrm{s}} .75$ & $-42^{\circ} 01^{\prime} 33^{\prime \prime} 9$ & $2.157 \pm 0.002$ & $20.35 \pm 0.21$ & 19 Nov. 2004 & $300 \mathrm{~V}$ & 400 & $1 . " 5$ & photometric \\
\hline \multirow[t]{3}{*}{ Q 0055-269 } & QNQ J00576-2626 & $00^{\mathrm{h}} 57^{\mathrm{m}} 36^{\mathrm{s}} .26$ & $-26^{\circ} 26^{\prime} 57^{\prime \prime} 1$ & $1.942 \pm 0.004$ & $20.73 \pm 0.19$ & 18 Nov. 2004 & $300 \mathrm{~V}$ & 300 & 1.2 & clear \\
\hline & QNQ J00582-2649 & $00^{\mathrm{h}} 58^{\mathrm{m}} 13^{\mathrm{s}} .94$ & $-26^{\circ} 49^{\prime} 19^{\prime}$. $^{2}$ & $2.572 \pm 0.003$ & $22.02 \pm 0.44$ & 18 Nov. 2004 & $300 \mathrm{~V}$ & 1800 & 0.9 & clear \\
\hline & QNQ J00583-2626 & $00^{\mathrm{h}} 58^{\mathrm{m}} 19^{\mathrm{s}} .50$ & $-26^{\circ} 26^{\prime} 12^{\prime \prime} 8$ & $2.720 \pm 0.006$ & $21.00 \pm 0.26$ & 18 Nov. 2004 & $300 \mathrm{~V}$ & 300 & 1.2 & clear \\
\hline \multirow[t]{2}{*}{ Q 0302-003 } & QNQ J03052-0016 & $03^{\mathrm{h}} 05^{\mathrm{m}} 15^{\mathrm{s}} .62$ & $-00^{\circ} 16^{\prime} 14^{\prime \prime} .4$ & $2.290 \pm 0.002$ & $20.05 \pm 0.06$ & 17 Nov. 2004 & $300 \mathrm{~V}$ & 300 & 0.7 & clear \\
\hline & QNQ J03052+0000 ${ }^{a}$ & $03^{\mathrm{h}} 05^{\mathrm{m}} 16^{\mathrm{s}} .95$ & $+00^{\circ} 00^{\prime} 43^{\prime \prime} 5$ & $2.808 \pm 0.004$ & $21.79 \pm 0.22$ & 17 Nov. 2004 & $300 \mathrm{~V}$ & 600 & 0.9 & clear \\
\hline \multirow[t]{10}{*}{ Q 0347-383 } & QNQ J03494-3814 & $03^{\mathrm{h}} 49^{\mathrm{m}} 24^{\mathrm{s}} .35$ & $-38^{\circ} 14^{\prime} 34^{\prime \prime} .2$ & $1.471 \pm 0.002$ & $19.60 \pm 0.04$ & 17 Nov. 2004 & $300 \mathrm{~V}$ & 60 & 0.8 & clear \\
\hline & QNQ J03500-3820 & $03^{\mathrm{h}} 50^{\mathrm{m}} 04^{\mathrm{s}} .26$ & $-38^{\circ} 20^{\prime} 51^{\prime \prime} .2$ & $1.819 \pm 0.002$ & $20.03 \pm 0.05$ & 17 Nov. 2004 & $300 \mathrm{~V}$ & 120 & 0.6 & clear \\
\hline & QNQ J03490-3812 & $03^{\mathrm{h}} 49^{\mathrm{m}} 03^{\mathrm{s}} .60$ & $-38^{\circ} 12^{\prime} 35^{\prime \prime} 8$ & $1.945 \pm 0.003$ & $21.66 \pm 0.19$ & 17 Nov. 2004 & $300 \mathrm{~V}$ & 600 & 0.0 & clear \\
\hline & QNQ J03496-3821 & $03^{\mathrm{h}} 49^{\mathrm{m}} 39^{\mathrm{s}} .31$ & $-38^{\circ} 21^{\prime} 34^{\prime \prime} .^{\prime} 1$ & $2.351 \pm 0.003$ & $19.43 \pm 0.03$ & 17 Nov. 2004 & $600 \mathrm{~B}$ & 300 & 0.7 & clear \\
\hline & QNQ J03496-3810 & $03^{\mathrm{h}} 49^{\mathrm{m}} 36^{\mathrm{s}} .28$ & $-38^{\circ} 10^{\prime} 02^{\prime \prime} 1$ & $2.433 \pm 0.003$ & $20.87 \pm 0.09$ & 17 Nov. 2004 & $600 \mathrm{~B}$ & 1800 & 0.8 & clear \\
\hline & QNQ J03495-3806 & $03^{\mathrm{h}} 49^{\mathrm{m}} 32^{\mathrm{s}} .61$ & $-38^{\circ} 06^{\prime} 45^{\prime \prime} 1$ & $2.475 \pm 0.003$ & $20.86 \pm 0.09$ & 17 Nov. 2004 & $600 \mathrm{~B}$ & 1800 & $\begin{array}{l}0.0 \\
0 ! 7\end{array}$ & clear \\
\hline & QNQ J03508-3812 & $03^{\mathrm{h}} 50^{\mathrm{m}} 50^{\mathrm{s}} \cdot 70$ & $-38^{\circ} 12^{\prime} 39^{\prime \prime} 0$ & $2.705 \pm 0.003$ & $20.86 \pm 0.06$ & 17 Nov. 2004 & $300 \mathrm{~V}$ & 360 & $0.0^{\prime \prime} 6$ & clear \\
\hline & QNQ J03503-3800 & $03^{\mathrm{h}} 50^{\mathrm{m}} 20^{\mathrm{s}} .01$ & $-38^{\circ} 00^{\prime} 03^{\prime \prime} 6$ & $2.734 \pm 0.003$ & $20.67 \pm 0.07$ & 17 Nov. 2004 & $600 \mathrm{~B}$ & 900 & 0.6 & clear \\
\hline & QNQ J03490-3825 & $03^{\mathrm{h}} 49^{\mathrm{m}} 02^{\mathrm{s}} .58$ & $-38^{\circ} 25^{\prime} 21^{\prime \prime} .5$ & $2.777 \pm 0.003$ & $20.52 \pm 0.06$ & 17 Nov. 2004 & $600 \mathrm{~B}$ & 1000 & 0.7 & clear \\
\hline & QNQ J03494-3826 & $03^{\mathrm{h}} 49^{\mathrm{m}} 28^{\mathrm{s}} .40$ & $-38^{\circ} 26^{\prime} 11^{\prime \prime} 6$ & $2.782 \pm 0.002$ & $20.97 \pm 0.07$ & 17 Nov. 2004 & $600 \mathrm{~B}$ & 1800 & 0.6 & clear \\
\hline \multirow[t]{3}{*}{ CTQ 0247} & QNQ J04061-4401 & $04^{\mathrm{h}} 06^{\mathrm{m}} 10^{\mathrm{s}} .38$ & $-44^{\circ} 01^{\prime} 00^{\prime} 0$ & $2.410 \pm 0.020$ & $19.35 \pm 0.05$ & 19 Nov. 2004 & $600 \mathrm{~B}$ & 900 & 1.0 & photometric \\
\hline & QNQ J04075-4416 & $04^{\mathrm{h}} 07^{\mathrm{m}} 35^{\mathrm{s}} 34$ & $-44^{\circ} 16^{\prime} 04^{\prime \prime} .1$ & $3.034 \pm 0.003$ & $21.75 \pm 0.19$ & 19 Nov. 2004 & $600 \mathrm{~B}$ & 2000 & $1{ }^{\prime \prime} 1$ & photometric \\
\hline & QNQ J04084-4420 & $04^{\mathrm{h}} 08^{\mathrm{m}} 29^{\mathrm{s}} .02$ & $-44^{\circ} 20^{\prime} 14^{\prime \prime} 4$ & $3.080 \pm 0.004$ & $22.88 \pm 0.41$ & 19 Nov. 2004 & $300 \mathrm{~V}$ & 1800 & 1.7 & photometric \\
\hline \multirow[t]{7}{*}{ Q 0420-388 } & QNQ J04217-3847 & $04^{\mathrm{h}} 21^{\mathrm{m}} 45^{\mathrm{s}} .70$ & $-38^{\circ} 47^{\prime} 44^{\prime \prime} .9$ & $0.771 \pm 0.002$ & $20.73 \pm 0.09$ & 18 Nov. 2004 & $600 \mathrm{~B}$ & 800 & 0.9 & clear \\
\hline & QNQ J04229-3831 & $04^{\mathrm{h}} 22^{\mathrm{m}} 59^{\mathrm{s}} .97$ & $-38^{\circ} 31^{\prime} 37^{\prime \prime} .1$ & $1.990 \pm 0.002$ & $19.98 \pm 0.06$ & 18 Nov. 2004 & $600 \mathrm{~B}$ & 400 & 0.8 & clear \\
\hline & QNQ J04222-3829 & $04^{\mathrm{h}} 22^{\mathrm{m}} 17^{\mathrm{s}} .31$ & $-38^{\circ} 29^{\prime} 33^{\prime \prime} 4$ & $2.168 \pm 0.003$ & $20.91 \pm 0.10$ & 18 Nov. 2004 & $300 \mathrm{~V}$ & 300 & 0.8 & clear \\
\hline & QNQ J04215-3857 & $04^{\mathrm{h}} 21^{\mathrm{m}} 34^{\mathrm{s}} .81$ & $-38^{\circ} 57^{\prime} 03^{\prime \prime} 4$ & $2.235 \pm 0.003$ & $22.23 \pm 0.27$ & 18 Nov. 2004 & $300 \mathrm{~V}$ & 1400 & 1.4 & clear \\
\hline & QNQ J04215-3854 & $04^{\mathrm{h}} 21^{\mathrm{m}} 32^{\mathrm{s}} .16$ & $-38^{\circ} 54^{\prime} 50^{\prime}{ }^{\prime} 6$ & $2.270 \pm 0.010$ & $19.49 \pm 0.04$ & 18 Nov. 2004 & $600 \mathrm{~B}$ & 200 & $0 ! 7$ & clear \\
\hline & QNQ J04212-3853 & $04^{\mathrm{h}} 21^{\mathrm{m}} 12.49$ & $-38^{\circ} 53^{\prime} 28^{\prime \prime} .5$ & $2.723 \pm 0.003$ & $20.08 \pm 0.06$ & 18 Nov. 2004 & $600 \mathrm{~B}$ & 1000 & 0.7 & clear \\
\hline & QNQ J04230-3853 & $04^{\mathrm{h}} 23^{\mathrm{m}} 01^{\mathrm{s}} \cdot 12$ & $-38^{\circ} 53^{\prime} 14^{\prime \prime} .5$ & $3.042 \pm 0.005$ & $21.95 \pm 0.15$ & 18 Nov. 2004 & $300 \mathrm{~V}$ & 1800 & $0 ! 7$ & clear \\
\hline HE $0940-1050$ & QNQ J09422-1117 & $09^{\mathrm{h}} 42^{\mathrm{m}} 13^{\mathrm{s}} .67$ & $-11^{\circ} 17^{\prime} 37^{\prime \prime} 5$ & $0.741 \pm 0.003$ & $20.10 \pm 0.07$ & 06 Apr. 2005 & $600 \mathrm{~B}$ & 420 & 0.9 & thin cirrus \\
\hline & QNQ J09437-1109 & $09^{\mathrm{h}} 43^{\mathrm{m}} 42^{\mathrm{s}} .67$ & $-11^{\circ} 09^{\prime} 47^{\prime \prime} 6$ & $1.456 \pm 0.002$ & $21.00 \pm 0.13$ & 06 Apr. 2005 & $300 \mathrm{~V}$ & 600 & $0 ! 8$ & thin cirrus \\
\hline & QNQ J09427-1108 & $09^{\mathrm{h}} 42^{\mathrm{m}} 44^{\mathrm{s}} .14$ & $-11^{\circ} 08^{\prime} 00^{\prime} \cdot 3$ & $1.517 \pm 0.004$ & $19.91 \pm 0.07$ & 12 May 2005 & $600 \mathrm{~B}$ & 300 & 1.2 & photometric \\
\hline & QNQ J09430-1108 & $09^{\mathrm{h}} 43^{\mathrm{m}} 00^{\mathrm{s}} .27$ & $-11^{\circ} 08^{\prime} 06^{\prime \prime} 3$ & $1.730 \pm 0.003$ & $21.03 \pm 0.12$ & 10 May 2005 & $300 \mathrm{~V}$ & 1800 & 0.7 & clear \\
\hline & QNQ J09424-1047 & $09^{\mathrm{h}} 42^{\mathrm{m}} 24^{\mathrm{s}} .17$ & $-10^{\circ} 47^{\prime} 58^{\prime \prime} .5$ & $1.971 \pm 0.003$ & $20.61 \pm 0.09$ & 12 May 2005 & $300 \mathrm{~V}$ & 240 & 0.7 & photometric \\
\hline & QNQ J09437-1057 & $09^{\mathrm{h}} 43^{\mathrm{m}} 45^{\mathrm{s}} .15$ & $-10^{\circ} 57^{\prime} 58^{\prime \prime} .8$ & $2.023 \pm 0.004$ & $20.64 \pm 0.10$ & 09 Jun. 2005 & $300 \mathrm{~V}$ & 240 & 0.6 & clear \\
\hline & QNQ J09435-1049 & $09^{\mathrm{h}} 43^{\mathrm{m}} 30^{\mathrm{s}} .04$ & $-10^{\circ} 49^{\prime} 58^{\prime \prime} .9$ & $2.216 \pm 0.003$ & $\begin{array}{l}20.04 \pm 0.10 \\
20.79 \pm 0.12\end{array}$ & 06 Apr. 2005 & $600 \mathrm{~B}$ & $\begin{array}{l}240 \\
720\end{array}$ & $\begin{array}{l}0.0 \\
0.9\end{array}$ & thin cirrus \\
\hline & QNQ J09425-1048 & $09^{\mathrm{h}} 42^{\mathrm{m}} 30^{\mathrm{s}} .58$ & $-10^{\circ} 48^{\prime} 50^{\prime}{ }^{\prime} 8$ & $2.325 \pm 0.005$ & 19.80 & 02 May 2005 & $600 \mathrm{~B}$ & 720 & 1.22 & thick cirrus \\
\hline & QNQ J09434-1053 & $09^{\mathrm{h}} 43^{\mathrm{m}} 24.21$ & $-10^{\circ} 53^{\prime} 32^{\prime \prime} 9$ & $2.760 \pm 0.003$ & $21.16 \pm 0.18$ & 12 May 2005 & $300 \mathrm{~V}$ & 1400 & 1,3 & photometric \\
\hline & QNQ J09427-1121 & $09^{\mathrm{h}} 42^{\mathrm{m}} 44^{\mathrm{s}} .42$ & $-11^{\circ} 21^{\prime} 38^{\prime \prime} 9$ & $2.963 \pm 0.003$ & $20.99 \pm 0.10$ & 19 Nov. 2004 & $600 \mathrm{~B}$ & $\begin{array}{l}1400 \\
1200\end{array}$ & 1.2 & photometric \\
\hline & & & & & & 05 Apr. 2005 & $600 \mathrm{~B}$ & 600 & 0.9 & thick cirrus \\
\hline & QNQ J09437-1052 & $09^{\mathrm{h}} 43^{\mathrm{m}} 42^{\mathrm{s}} .99$ & $-10^{\circ} 52^{\prime} 31^{\prime \prime} .7$ & $3.018 \pm 0.003$ & $20.78 \pm 0.09$ & 19 Nov. 2004 & $600 \mathrm{~B}$ & 1200 & 1.3 & photometric \\
\hline & & & & & & 06 Apr 2005 & $600 \mathrm{~B}$ & 1200 & 0,5 & thick cirrus \\
\hline CTQ 0460 & QNQ J10399-2321 & $10^{\mathrm{h}} 39^{\mathrm{m}} 58^{\mathrm{s}} .77$ & $-23^{\circ} 21^{\prime} 40^{\prime} \cdot 3$ & $2.216 \pm 0.004$ & $20.73 \pm 0.16$ & 07 May 2005 & $600 \mathrm{~B}$ & 900 & $\begin{array}{l}0.7 \\
0.6\end{array}$ & thin cirrus \\
\hline & QNQ J10388-2258 & $10^{\mathrm{h}} 38^{\mathrm{m}} 50^{\mathrm{s}} .12$ & $-22^{\circ} 58^{\prime} 08^{\prime \prime} .9$ & $2.326 \pm 0.003$ & $19.63 \pm 0.07$ & 28 Jun. 2005 & $600 \mathrm{~B}$ & 420 & $\begin{array}{l}0.0 \\
0.7\end{array}$ & thin cirrus \\
\hline & QNQ J10385-2317 & $10^{\mathrm{h}} 38^{\mathrm{m}} 31^{\mathrm{s}} .54$ & $-23^{\circ} 17^{\prime} 55^{\prime \prime} 3$ & $3.099 \pm 0.004$ & $21.28 \pm 0.11$ & 09 Jun. 2005 & $600 \mathrm{~B}$ & 1800 & 0.6 & clear \\
\hline BR 1117-1329 & QNQ J11208-1345 & $11^{\mathrm{h}} 20^{\mathrm{m}} 48.50$ & $-13^{\circ} 45^{\prime} 35^{\prime \prime} 6$ & $1.893 \pm 0.002$ & $20.10 \pm 0.08$ & 27 Jun. 2005 & $300 \mathrm{~V}$ & 180 & 0.9 & thin cirrus \\
\hline & QNQ J11205-1343 & $11^{\mathrm{h}} 20^{\mathrm{m}} 34^{\mathrm{s}} .09$ & $-13^{\circ} 43^{\prime} 28^{\prime \prime} 9$ & $1.910 \pm 0.002$ & $19.84 \pm 0.07$ & 27 Jun. 2005 & $300 \mathrm{~V}$ & 180 & 0.8 & thin cirrus \\
\hline & QNQ J11197-1340 & $11^{\mathrm{h}} 19^{\mathrm{m}} 46^{\mathrm{s}} .71$ & $-13^{\circ} 40^{\prime} 47^{\prime \prime} 1$ & $2.220 \pm 0.004$ & $18.32 \pm 0.04$ & 27 Jun. 2005 & $600 \mathrm{~B}$ & 420 & $\begin{array}{l}0.0 \\
0 ! 9\end{array}$ & thin cirrus \\
\hline & QNQ J11192-1334 & $11^{\mathrm{h}} 19^{\mathrm{m}} 12^{\mathrm{s}} .37$ & $-13^{\circ} 34^{\prime} 05^{\prime \prime} 5$ & $3.252 \pm 0.005$ & $22.55 \pm 0.40$ & 27 Jun. 2005 & $300 \mathrm{~V}$ & $\begin{array}{l}420 \\
1600\end{array}$ & $0.0^{\prime} 8$ & thin cirrus \\
\hline BR 1202-0725 & QNQ J12059-0754 & $12^{\mathrm{h}} 05^{\mathrm{m}} 55^{\mathrm{s}} .33$ & $-07^{\circ} 54^{\prime} 33^{\prime \prime} 77$ & $0.773 \pm 0.001$ & $20.40 \pm 0.20$ & 06 May 2005 & $600 \mathrm{~B}$ & 900 & $\begin{array}{l}0.0 \\
1.5\end{array}$ & clear \\
\hline & QNQ J12062-0727 & $12^{\mathrm{h}} 06^{\mathrm{m}} 14^{\mathrm{s}} .24$ & $-07^{\circ} 27^{\prime} 41^{\prime \prime} 1$ & $1.478 \pm 0.003$ & $20.46 \pm 0.08$ & 29 Jun. 2005 & $300 \mathrm{~V}$ & 180 & 0.9 & thin cirrus \\
\hline & QNQ J12061-0745 & $12^{\mathrm{h}} 06^{\mathrm{m}} 08^{\mathrm{s}} .91$ & $-07^{\circ} 45^{\prime} 49^{\prime \prime} 8$ & $1.730 \pm 0.002$ & $19.15 \pm 0.06$ & 06 May 2005 & $600 \mathrm{~B}$ & 600 & 1.5 & clear \\
\hline Q 1209+093 & QNQ J12124+0851 & $12^{\mathrm{h}} 12^{\mathrm{m}} 29^{\mathrm{s}} .08$ & $+08^{\circ} 51^{\prime} 58^{\prime \prime} 9$ & $0.729 \pm 0.003$ & $19.61 \pm 0.05$ & 28 Jun. 2005 & $600 \mathrm{~B}$ & 300 & $\begin{array}{l}1.3 \\
0 !\end{array}$ & thin cirrus \\
\hline & QNQ J12111+0906 & $12^{\mathrm{h}} 11^{\mathrm{m}} 06^{\mathrm{s}} .59$ & $+09^{\circ} 06^{\prime} 43^{\prime \prime} 6$ & $2.534 \pm 0.002$ & $21.17 \pm 0.15$ & 29 Jun. 2005 & $600 \mathrm{~B}$ & 2400 & $0 ! 7$ & thin cirrus \\
\hline PKS 2126-158 & QNQ J21294-1521 & $21^{\mathrm{h}} 29^{\mathrm{m}} 29^{\mathrm{s}} .18$ & $-15^{\circ} 21^{\prime} 57^{\prime \prime} \cdot 9$ & $0.580 \pm 0.001$ & 18.38 & 04 May 2005 & $600 \mathrm{~B}$ & 180 & 0.8 & thin cirrus \\
\hline & QNQ J21291-1524A & $21^{\mathrm{h}} 29^{\mathrm{m}} 10^{\mathrm{s}} .09$ & $-15^{\circ} 24^{\prime} 45^{\prime \prime} 1$ & $0.782 \pm 0.001$ & $21.81 \pm 0.30$ & 04 May 2005 & $300 \mathrm{~V}$ & 2400 & 0.9 & thin cirrus \\
\hline & QNQ J21297-1536 & $21^{\mathrm{h}} 29^{\mathrm{m}} 46^{\mathrm{s}} .75$ & $-15^{\circ} 36^{\prime} 51^{\prime \prime} 9$ & $1.509 \pm 0.003$ & $20.50 \pm 0.13$ & 06 May 2005 & $300 \mathrm{~V}$ & 180 & $1 " .3$ & clear \\
\hline & QNQ J21286-1528 & $21^{\mathrm{h}} 28^{\mathrm{m}} 40^{\mathrm{s}} .43$ & $-15^{\circ} 28^{\prime} 15^{\prime \prime} 7$ & $1.925 \pm 0.005$ & $19.79 \pm 0.10$ & 06 May 2005 & $300 \mathrm{~V}$ & 120 & $1 . " 6$ & clear \\
\hline & QNQ J21291-1524B & $21^{\mathrm{h}} 29^{\mathrm{m}} 10^{\mathrm{s}} .85$ & $-15^{\circ} 24^{\prime} 23^{\prime \prime} 7$ & $2.480 \pm 0.030$ & $20.33 \pm 0.12$ & 04 May 2005 & $600 \mathrm{~B}$ & 720 & 1.0 & thin cirrus \\
\hline & QNQ J21301-1533 & $21^{\mathrm{h}} 30^{\mathrm{m}} 07^{\mathrm{s}} .46$ & $-15^{\circ} 33^{\prime} 20^{\prime} 9$ & $3.487 \pm 0.003$ & $21.94 \pm 0.32$ & 05 May 2005 & $600 \mathrm{~B}$ & 2200 & 0.9 & clear \\
\hline Q 2139-4434 & QNQ J21434-4432 & $21^{\mathrm{h}} 43^{\mathrm{m}} 25^{\mathrm{s}} .26$ & $-44^{\circ} 32^{\prime} 11^{\prime \prime} .0$ & $2.709 \pm 0.004$ & $19.33 \pm 0.06$ & 05 May 2005 & $600 \mathrm{~B}$ & 540 & 1.1 & clear \\
\hline HE 2243-6031 & QNQ J22454-6020 & $22^{\mathrm{h}} 45^{\mathrm{m}} 27^{\mathrm{s}} .22$ & $-60^{\circ} 20^{\prime} 25^{\prime \prime} .2$ & $1.984 \pm 0.015$ & $20.94 \pm 0.29$ & 19 Nov. 2004 & $300 \mathrm{~V}$ & 300 & $1 . " 6$ & photometric \\
\hline & QNQ J22455-6015 & $22^{\mathrm{h}} 45^{\mathrm{m}} 34^{\mathrm{s}} .64$ & $-60^{\circ} 15^{\prime} 45^{\prime \prime} 9$ & $2.036 \pm 0.002$ & $21.74 \pm 0.42$ & 19 Nov. 2004 & $300 \mathrm{~V}$ & 800 & 1.2 & photometric \\
\hline & QNQ J22460-6024 & $22^{\mathrm{h}} 46^{\mathrm{m}} 01^{\mathrm{s}} .23$ & $-60^{\circ} 24^{\prime} 57^{\prime \prime} .5$ & $2.041 \pm 0.003$ & $21.25 \pm 0.33$ & 19 Nov. 2004 & $300 \mathrm{~V}$ & 1800 & 1.2 & photometric \\
\hline & QNQ J22454-6011 & $22^{\mathrm{h}} 45^{\mathrm{m}} 29^{\mathrm{s}} .42$ & $-60^{\circ} 11^{\prime} 17^{\prime \prime} 1$ & $2.324 \pm 0.003$ & $19.36 \pm 0.06$ & 17 Nov. 2004 & $600 \mathrm{~B}$ & 300 & $0 ! 9$ & clear \\
\hline & QNQ J22463-6009 & $22^{\mathrm{h}} 46^{\mathrm{m}} 18^{\mathrm{s}} .47$ & $-60^{\circ} 09^{\prime} 02^{\prime \prime} 5$ & $2.329 \pm 0.003$ & $19.71 \pm 0.09$ & 17 Nov. 2004 & $600 \mathrm{~B}$ & 500 & 1.0 & clear \\
\hline & QNQ J22484-6002 & $22^{\mathrm{h}} 48^{\mathrm{m}} 29^{\mathrm{s}} .20$ & $-60^{\circ} 02^{\prime} 19^{\prime}$. $^{\prime}$ & $3.586 \pm 0.002$ & $20.97 \pm 0.20$ & 17 Nov. 2004 & $600 \mathrm{~B}$ & 600 & 1.0 & clear \\
\hline & & & & & & 19 Nov. 2004 & $600 \mathrm{~B}$ & 600 & $1 " .4$ & photometric \\
\hline
\end{tabular}


G. Worseck et al.: A slitless spectroscopic survey for quasars near quasars, Online Material $p 19$

Table 2. continued.

\begin{tabular}{|c|c|c|c|c|c|c|c|c|c|c|}
\hline Field & QSO & $\alpha(\mathrm{J} 2000)$ & $\delta(\mathrm{J} 2000)$ & $z$ & $B$ & Night & Grism & $t_{\exp }[\mathrm{s}]$ & Seeing & Transparency \\
\hline \multirow[t]{11}{*}{ HE $2347-4342$} & QNQ J23510-4336 & $23^{\mathrm{h}} 51^{\mathrm{m}} 05^{\mathrm{s}} .50$ & $-43^{\circ} 36^{\prime} 57^{\prime \prime} .2$ & $0.720 \pm 0.002$ & $20.74 \pm 0.27$ & 19 Nov. 2004 & $300 \mathrm{~V}$ & 1200 & $1 " .3$ & photometric \\
\hline & QNQ J23507-4319 & $23^{\mathrm{h}} 50^{\mathrm{m}} 44^{\mathrm{s}} .97$ & $-43^{\circ} 19^{\prime} 26^{\prime \prime} 0$ & $0.850 \pm 0.003$ & $19.90 \pm 0.07$ & 17 Nov. 2004 & $600 \mathrm{~B}$ & 360 & 0.7 & clear \\
\hline & QNQ J23507-4326 & $23^{\mathrm{h}} 50^{\mathrm{m}} 45^{\mathrm{s}} \cdot 39$ & $-43^{\circ} 26^{\prime} 37^{\prime \prime} 0$ & $1.635 \pm 0.003$ & $21.05 \pm 0.14$ & 17 Nov. 2004 & $300 \mathrm{~V}$ & 200 & $1{ }^{\prime \prime} 0$ & clear \\
\hline & QNQ J23509-4330 & $23^{\mathrm{h}} 50^{\mathrm{m}} 54^{\mathrm{s}} .80$ & $-43^{\circ} 30^{\prime} 42^{\prime \prime} .2$ & $1.762 \pm 0.004$ & $18.23 \pm 0.03$ & 17 Nov. 2004 & $600 \mathrm{~B}$ & 300 & 0.7 & clear \\
\hline & QNQ J23502-4334 & $23^{\mathrm{h}} 50^{\mathrm{m}} 16^{\mathrm{s}} .18$ & $-43^{\circ} 34^{\prime} 14^{\prime \prime} 7$ & $1.763 \pm 0.003$ & $18.95 \pm 0.04$ & 17 Nov. 2004 & $300 \mathrm{~V}$ & 60 & 0.7 & clear \\
\hline & QNQ J23503-4328 & $23^{\mathrm{h}} 50^{\mathrm{m}} 21^{\mathrm{s}} .55$ & $-43^{\circ} 28^{\prime} 43^{\prime \prime} 7$ & $2.282 \pm 0.003$ & $20.66 \pm 0.11$ & 17 Nov. 2004 & $300 \mathrm{~V}$ & 400 & 0.7 & clear \\
\hline & QNQ J23495-4338 & $23^{\mathrm{h}} 49^{\mathrm{m}} 34^{\mathrm{s}} .53$ & $-43^{\circ} 38^{\prime} 08^{\prime \prime} .7$ & $2.690 \pm 0.006$ & $20.21 \pm 0.17$ & 19 Nov. 2004 & $300 \mathrm{~V}$ & 360 & 1.2 & photometric \\
\hline & QNQ J23511-4319 & $23^{\mathrm{h}} 51^{\mathrm{m}} 09^{\mathrm{s}} .44$ & $-43^{\circ} 19^{\prime} 41^{\prime \prime} 6$ & $3.020 \pm 0.004$ & $21.00 \pm 0.14$ & 17 Nov. 2004 & $600 \mathrm{~B}$ & 1000 & $1 " .1$ & clear \\
\hline & QNQ J23514-4339 & $23^{\mathrm{h}} 51^{\mathrm{m}} 25.54$ & $-43^{\circ} 39^{\prime} 02^{\prime \prime} 9$ & $3.240 \pm 0.004$ & $21.57 \pm 0.29$ & 17 Nov. 2004 & $300 \mathrm{~V}$ & 1400 & $1 " 2$ & clear \\
\hline & QNQ J23503-4317 & $23^{\mathrm{h}} 50^{\mathrm{m}} 21^{\mathrm{s}} .94$ & $-43^{\circ} 17^{\prime} 30^{\prime} .0$ & $3.542 \pm 0.005$ & $21.94 \pm 0.62$ & 19 Nov. 2004 & $300 \mathrm{~V}$ & 1800 & $1 . .2$ & photometric \\
\hline & & & & & & 19 Nov. 2004 & $600 \mathrm{~B}$ & 1800 & $1 " .2$ & photometric \\
\hline
\end{tabular}

${ }^{a}$ Due to imperfect astrometry the position of QNQ J03052+0000 given in Paper I is incorrect by $\simeq 3^{\prime \prime}$. Here we give the coordinates measured in the SDSS. 